\title{
Three Essays on Corporate Financial Constraints
}

by

Kwabena Antwi Boasiako

A thesis submitted to the Victoria University of Wellington in fulfillment of the requirements for the degree of Doctor of Philosophy in Finance.

Victoria University of Wellington

June 2021 


\section{Abstract}

This thesis is composed of three self-contained empirical essays in corporate finance, with the first two exploring the financial policy and credit risk implications of data breaches, and the third examining whether financing influences the sensitivity of cash and investment to asset tangibility.

In the first essay, we contribute to the growing debate on cybersecurity risks and how firms can insulate themselves, at least partially, from the adverse effects of data breach risks. Specifically, we examine the effects of data breach disclosure laws and the subsequent disclosure of data breaches on the cash policies of corporations in the United States (U.S.). Exploiting a series of natural experiments regarding staggered state-level data breach disclosure laws, we find that the passage of mandatory disclosure laws leads to an increase in cash holdings. Our finding suggests that mandatory data breach disclosure laws increase the ex ante risks related to data breaches, hence, firms hold on to more cash as a precautionary motive. Further, we find firms that suffer data breaches adjust their financial policies by holding more cash as well as decreasing external finance and investment.

The second essay examines the impact of data breaches on firm credit risk. Using firmlevel credit ratings and credit default swap (CDS) spreads to proxy for credit risk, we find that data breaches lead to increases in firm credit risk. Firms exposed to data breaches are more likely to experience credit rating downgrades and an increase in the CDS spread of traded bonds. Also, firms who suffer data breaches report lower sales and ROA, experience an increase in financial distress, and conditional on a data breach incident, 
the likelihood of a future data breach increases. Lastly, these effects are magnified for firms with low-interest coverage ratios.

In the third essay, using the financial deregulation of seasoned equity issuance in the U.S. as an exogenous shock to access to equity markets, I investigate the influence of financing on the sensitivity of cash and investment to asset tangibility. I show that financing dampens the sensitivity of cash and investment to asset tangibility and promotes investment and firm growth. This provides evidence that public firms even in well-developed financial markets such as the U.S., benefit from financial deregulation that removes barriers to external equity financing, shedding light on the role of financial markets in fostering growth. 


\section{Acknowledgments}

I extend my sincerest and profound gratitude to Michael Keefe for his wonderful supervision throughout my Ph.D. journey. His advice, input, and unwavering support immensely helped me to improve the quality of this dissertation. He is the kind of supervisor any Ph.D. student would be so blessed and lucky to have. Under his supervision, the first essay was published in the European Financial Management, the second essay is under review at the Journal of Banking and Finance, and the third essay received an R\&R editorial decision from the International Review of Financial Analysis. I am most grateful, Michael.

To my beloved wife, Adwoa, special thanks to you for the endless love, unyielding support, and the countless sacrifices you made throughout this journey. To my wee

princess, Enijie, your coming into this world gave me a renewed sense of purpose. Also, I am very much thankful to my parents and parents-in-law for their love and support.

My sincere thanks to all the members of Christ for Salvation Ministries in Wellington, in particular, Pastor Willard and Pastor Peace, who have in no small way positively influenced my life. Finally, to all the friends who became family, I am most grateful. 


\section{Contents}

1 Data breaches and corporate liquidity management 9

1.1 Introduction . . . . . . . . . . . . . . . . . . . . . 10

1.2 Background . . . . . . . . . . . . . . . . . . 17

1.2.1 State-level data breach disclosure laws in the United States . . . . 17

1.2 .2 Related literature . . . . . . . . . . . . . . . . . . 19

1.3 Data and Methodology . . . . . . . . . . . . . . . 20

1.3.1 Data Collection . . . . . . . . . . . . . . 20

1.3.2 Estimation Technique . . . . . . . . . . . . . . . . 21

1.3.3 Variables......................... 22

1.4 State-Level Disclosure Laws and Corporate Cash holdings . . . . . . . . 23

1.4.1 Baseline Regression Results . . . . . . . . . . . . . . . . . 23

1.4.2 Falsification Test . . . . . . . . . . . . . 25

1.4.3 Role of Financial Constraints . . . . . . . . . . . . . . . . 26

1.5 Effects of Actual Data Breaches . . . . . . . . . . . . . . . . . . 27

1.5.1 Association between Data Breaches and Observable Firm Charac-

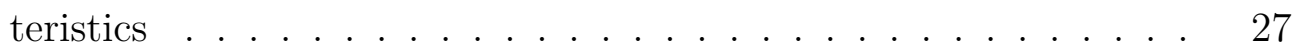

1.5.2 Data Breaches and Cash Holdings . . . . . . . . . . . . . . 28

1.5.3 Mechanisms ........................ 29

1.6 Conclusion . . . . . . . . . . . . . . . . . . . . . . . 32

2 Data breaches and firm credit risk 45 
2.1 Introduction . . . . . . . . . . . . . . . . . . 46

2.2 Hypotheses . . . . . . . . . . . . . . . . . . . . . . . 51

2.3 Data and Sample Selection . . . . . . . . . . . . . . . 52

2.3.1 Data collection . . . . . . . . . . . . . . 52

2.3.2 Matched sample . . . . . . . . . . . . . . 54

2.3.3 Variables.......................... 55

2.4 Research Design . . . . . . . . . . . . . . . . . . . 56

2.5 Empirical Results . . . . . . . . . . . . . . . . . . . . 60

2.5.1 Data breaches and credit ratings . . . . . . . . . . . 60

2.5.2 Data breaches and credit default swap (CDS) spreads . . . . . . . 62

2.5.3 The impact of interest coverage . . . . . . . . . . . . 63

2.5.4 Mechanism ..................... 64

2.5.5 Robustness tests . . . . . . . . . . . . . 66

2.6 Conclusion . . . . . . . . . . . . . . . . . . . . . 67

3 Does financing influence the sensitivity of cash and investment to asset $\begin{array}{ll}\text { tangibility? } & 84\end{array}$

3.1 Introduction . . . . . . . . . . . . . . . . . . 85

3.2 Institutional detail: deregulation of equity issuance in the US . . . . . . 94

3.3 Sample construction . . . . . . . . . . . . . . . . . . . . . 95

3.4 Empirical strategy f . . . . . . . . . . . . . . . . . . . 97

3.5 Empirical analysis . . . . . . . . . . . . . . . . . . . . . . . . 99

3.5.1 Financing, asset tangibility, and cash . . . . . . . . . . . 99

3.5.2 Information asymmetry and tech intensiveness . . . . . . . . . . 101

3.5.3 Financing and the investment-cash flow sensitivity . . . . . . . . . 102

3.5.4 Financing, asset tangibility, and investment . . . . . . . . . 103

3.5.5 Financing and the quality of investment decisions . . . . . . . . . 104

3.5.6 Financing and firm asset growth . . . . . . . . . . . 105 
3.5.7 Robustness test . . . . . . . . . . . . . . . 106

3.6 Conclusion . . . . . . . . . . . . . . . . . . . 107 


\section{List of Tables}

1.1 Summary statistics . . . . . . . . . . . . . . . . 36

1.2 State-level disclosure laws and cash holdings . . . . . . . . . . . . . 37

1.3 Randomization of state-level data breach disclosure laws . . . . . . . . . 38

1.4 Disclosure laws and corporate cash holdings: Role of financial constraints 39

1.5 Association between data breaches and observable firm characteristics . . 40

1.6 Data breaches and cash holdings . . . . . . . . . . . . . . . 41

1.7 Data breaches and external financing . . . . . . . . . . . . . . . 42

1.8 Data breaches and corporate investment . . . . . . . . . . . 43

1.9 Variable definitions . . . . . . . . . . . . . . . . . . . . 44

2.1 Summary statistics . . . . . . . . . . . . . . . . . . . . 72

2.2 Data breaches and credit ratings . . . . . . . . . . . . . 73

2.3 Data breaches and probability of speculative grade rating . . . . . . . 74

2.4 CDS market reaction to data breaches . . . . . . . . . . . . 75

2.5 Data breaches and CDS spread . . . . . . . . . . . 76

2.6 Interest coverage and the effect of data breaches on credit ratings . . . . 77

2.7 Interest coverage and the effect of data breaches on CDS spread . . . . 78

2.8 Data breach, profitability, and distress . . . . . . . . . . . 79

2.9 Conditional likelihood of future data breaches . . . . . . . . . . . . 80

2.10 Robustness: CDS market reaction to data breaches . . . . . . . . . . 80

2.11 Robustness: Data breach and profitability . . . . . . . . . . . 81

2.12 Robustness: Interest coverage and the effect of data breaches on credit risk 82 
3.1 Summary statistics: comparison of key characteristics . . . . . . . . . . 111

3.2 Financing and cash-tangibility sensitivity . . . . . . . . . . . . . . 112

3.3 Placebo: lower and upper public float cutoffs . . . . . . . . . . . . . . . 113

3.4 Information asymmetry and tech intensiveness . . . . . . . . . . . . . 114

3.5 Financing and the investment-cash flow sensitivity . . . . . . . . . . 115

3.6 Financing and investment-tangibility sensitivity . . . . . . . . . 116

3.7 Financing and investment efficiency . . . . . . . . . . . . . . . . . . . 117

3.8 Financing and fixed asset growth f . . . . . . . . . . . . 118

3.9 Robustness check: financing and sensitivity of cash and investment to asset tangibility . . . . . . . . . . . . . . . . . . . 119

3.10 Variable definitions . . . . . . . . . . . . . . . 120 
Chapter 1

Data breaches and corporate

liquidity management 


\subsection{Introduction}

Data are among a corporation's most valuable assets. Financial policies associated with data breaches are important, particularly in the wake of the increase in the frequency and severity of data breaches and the rising wave of cybersecurity regulations. ${ }^{1}$ With billions of sensitive corporate and consumer data compromised and thousands of breaches disclosed each year, data breaches are a growing concern for corporate boards in the United States as they face mounting regulatory pressure to cope with cybersecurity risks. For example, Fuhrmans (2017) asserts, "Cyber threats have zoomed to the top of chief executives' worry lists for fear a data breach could cost them their jobs and take down their businesses." Moreover, the fallout from other data breaches, such as the attacks on Equifax, Home Depot, Yahoo, and Target, provide compelling evidence that the risks to data security matter at the highest corporate levels. Although cybersecurity regulations and data breaches have attracted huge media attention and are now key boardroom issues of concern to corporate executives, they have received little attention in the empirical finance literature.

In this paper, we contribute to the growing debate on cybersecurity risks and how firms can insulate themselves, at least partially, from the adverse effects of data breach risks. A data breach incident takes many forms such as hacking, crimeware, cyber-espionage, denial of service, insider and privilege misuse, payment card skimmers, point of sale intrusions, physical theft and loss, and web application attacks. The occurrence of a data breach could adversely affect corporations through breach remediation costs, regulatory action and fines, higher cost of capital, litigation, loss of customers and reduced revenue, loss of intellectual property and strategic knowledge. Having incident response teams, encryption, employee training, and many other mechanisms could help firms lower the cost of data breaches. However, anecdotal evidence shows that breached firms have to spend considerably to overhaul their cyber security and prepare for potential expenses

\footnotetext{
${ }^{1}$ For instance, the European Union's General Data Protection Regulation went into effect on May 25, 2018.
} 
such as litigation expenses. ${ }^{2}$ The direct and indirect effects of these consequences can be felt in both the short-term and long-term. Therefore, in this paper, we explore several questions related to data breaches and financial outcomes and policies. For instance, do laws that mandate the disclosure of data breaches and compel corporations to account for their data insecurity affect corporate liquidity management? Do firms change their financial policies to reflect changes in risk exposure due to a successful cyberattack on them? Do firms handle the direct and indirect costs of data breaches by changing their financial policies? To address these questions, we exploit plausibly exogenous state-level variation in data breach disclosure laws and subsequently disclosed data breaches.

Before 2002, US firms were not obligated to disclose data breaches. During the period from 2002 to 2016, state-level disclosure laws mandating firms to publicly disclose data breach incidents were passed in almost all the US states. In 2002, California became the first state to pass a data breach disclosure law. Since the passage of the California law, the other 49 states passed similar laws. While the details of the disclosure laws vary across the states, their central theme is consistent: firms should publicly disclose data breaches. Since mandatory state-level disclosure laws prevent firms from sweeping data breaches under the rug, the mandatory breach disclosure requirement transforms private information about the practices of firms into public information, causing firms to face higher risks and costs relating to publicly disclosed data breaches. Even though the likelihood of experiencing a data breach could remain unchanged, a firm must publicly disclose data breaches, which significantly increases its risks and costs. This institutional background offers a unique avenue through which we can examine the impact of mandatory data breach laws and disclosed breaches on corporate financial policies.

Exposure to and disclosure of a data security breach create direct and indirect costs. The disclosure of data breaches can potentially subject firms to regulatory investigations and fines, litigation, media scrutiny and reputation damage, customer loss, revenue decline, and increased cash flow risks and possibly threaten a firm's bottom line, as well as

\footnotetext{
${ }^{2}$ For instance, Equifax in its 2019 earnings release disclosed that the 2017 data breach incident had cost the firm $\$ 1.4$ billion.
} 
shareholder value, among other things. In short, mandatory data breach disclosure laws increase a firm's ex ante risk of incurring future costs related to disclosed breaches. A corporate policy that could be particularly sensitive to the increased risks and costs of data breaches following the passage of a mandatory disclosure law is the decision to accumulate cash. Prior literature underlines the relation between risk and cash holdings. Froot and Stein (1998) model the risk management choices of firms in situations in which some risks are not perfectly hedgeable. Froot and Stein (1998) find that, when it is difficult to completely hedge a particular type of risk, firms resort to increasing their cash holdings. Similarly, if hedging is impossible or when firms face high hedging transaction costs for a particular type of risk, an alternative way to minimize risk exposure is to accumulate cash (Brav et al. (2005); Han and Qiu (2007); Riddick and Whited (2009)). Bates et al. (2009) highlight the importance of the precautionary motive in determining corporate cash holdings. Consistent with this argument, Ucar (2019) reports that firms located in areas with a more creative culture have higher levels of risk exposure and accumulate more cash, consistent with the precautionary motive. Acharya et al. (2012) examine the relationship between cash holdings and credit risk and find that riskier firms accumulate more cash. Chen et al. (2020) exploit a unique setting in China and show that firms with lower internal control quality are more likely to hold more cash. Additionally, Arena and Julio (2015) find that firms facing litigation risks significantly increase their holdings of cash. Romanosky et al. (2014) empirically investigate the impact of data breaches on litigation risk, and the outcomes of data breach litigation. They show that, between 2000 and 2011, the overall settlement rate of known federal lawsuits due to data breaches was around 50\%, and defendants even settle $30 \%$ more often when plaintiffs allege financial loss from a data breach or when faced with a class action data breach suit.

We hypothesize that data breach risk exposure is a significant determinant of a firm's decision to accumulate cash. Following the mandatory data breach disclosure laws, we advance that firms can ex ante adjust cash policies to help hedge against the increasing risks and future costs related to data breaches. Thus, we expect that, following the 
passage of mandatory state-level data breach disclosure laws, all else being equal, a firm with higher exposure to risk from data breach disclosures will increase its cash holdings. Similarly, firms that are victims of successful data breaches increase their cash holdings in anticipation of settlement costs related to the disclosed data breach.

Firms can obtain insurance contracts to protect themselves from the direct costs of data breaches. However, in practice, cyber insurance does not cover consequential damages; hence, it is difficult (if not impossible) to completely hedge or insure the majority of the costs associated with a disclosed data breach incident (especially indirect or hardto-quantify costs such as reputation damage, loss of value of intellectual property, or customer loss). ${ }^{3}$ Importantly, no cyber insurance contract provides complete or full coverage prevention and/or incident response services. For instance, no cyber insurance policy provides coverage for reputational costs, which, according to the 2018 IBM/Ponemon Institute data breach studies, constitutes the largest indirect per capita cost and the largest data breach cost category in the United States. In many cases, determining and obtaining the right policies can be challenging due to difficulties in understanding and assessing vulnerabilities to breaches. Therefore, practitioners, advance that an efficient way firms can manage the potential risks and costs of a data breach is to increase cash reserves. For instance, Deloitte's 2018 risk management, strategy, and analysis report argue that the creation of sufficient cash reserves improves a firm's ability to quickly recover and survive data breaches.

To identify the effect of data breach disclosure laws on firm cash holdings, we construct tests that exploit time-varying exogenous changes in state-level data breach disclosure laws. The staggered timing of the passage of the state-level disclosure laws plausibly provides a natural experiment framework to empirically explore how these regulatory changes affect corporate cash policies. Similar to previous studies (Beck et al. (2010); Francis et al. (2014)), we use a difference-in-differences approach to examine how firms change their cash policies in response to changes in state-level data breach disclosure

\footnotetext{
${ }^{3}$ Some intangible damages take months to emerge (varying across firms), which makes a standard process of evaluation difficult to create.
} 
laws. Our baseline regression results indicate an economically important positive relation between state-level data breach disclosure laws and corporate cash holdings. First, the results are robust to the exclusion of the financial crisis period, to the exclusion of firms based in California, which account for $18 \%$ of observations in our sample, and to the re-estimation of our baseline specification in first differences.

Second, while the staggered timing of the passage of the disclosure laws provides exogenous changes to data security reporting and transparency, unobservable shocks coinciding with state-level data breach disclosure laws could be the primary cause of changes in cash holdings. If this is the case, then the changes in corporate cash holdings we attribute to state-level disclosure laws reflect mere association, rather than causality. Our strategy of using multiple shocks helps isolate the impact of disclosure laws on cash holdings from other confounding factors, thus mitigating omitted variable concerns. Additionally, the timing and passage of state-level disclosure laws are beyond the control of any individual firm; that is, firms have no influence or control over the years in which disclosure laws are passed. Even if the enactment of various state-level data breach disclosure laws is anticipated, firms could have started changing their financial policies prior to the laws' passage, which should disfavor us finding any impact of the changes in the state-level disclosure laws on our outcome of interest.

Nevertheless, we address this possibility by conducting placebo or falsification tests following an approach similar to that of Cornaggia et al. (2015). We randomly allocate the various states to the distribution of data breach disclosure law years. This preserves the correct distribution of the disclosure law years but randomly assigns the states to the disclosure law years. We expect our re-estimated results to be weaker if unobservable shocks do not exist in our testing framework; that is, falsely assumed disclosure law events should have no effect on cash holdings. Indeed, our randomized disclosure law estimation results show no effect on corporate cash holdings. The non-result in this test discounts an omitted variable bias. 
After providing robust evidence of an aggregate increase in corporate cash holdings following the passage of various state-level data breach disclosure laws, we turn to the analysis of actual data breaches. We first examine the association between data breaches and certain observable firm characteristics. Our findings, suggestive and exploratory and not definitive determinants of data breaches, show that data breaches are more likely for large and old firms, financially constrained firms, and high-growth firms.

Next, we test the impact of exposure to and the disclosure of data breaches on changes in corporate cash holdings. We find that firms increase their cash holdings in response to a data breach incident. To test that the results are not driven by differences in characteristics between breached and non-breached firms, we follow Hainmueller and $\mathrm{Xu}$ (2013) and use the entropy balancing approach, and we still find a positive relation between data breaches and corporate cash holdings. Although we find a positive relation between data breaches and cash holdings, one could argue that the increase in cash balances following disclosure laws should be used up by breached firms in meeting the cash outlays associated with the breach incident. However, while firms build up their cash reserves after the enactment of the laws, it is possible breached firms build up cash because of an unusually bad breach incident. Thus, a firm's cash needs are plausibly unanticipated when an unusually bad breach occurs, and they react by increasing their cash holdings. We examine this possibility by running a test that relates the ex post cash build up to the severity of data breaches. As a proxy for the expected costs of unusually bad breach incidents, we use the stock market reaction to the breach announcement. Specifically, we use an event study methodology to estimate the cumulative abnormal returns (CARs) around the data breach announcement. We then rank the CARs into terciles and estimate three levels of data breach severity. The results show that firms with severe breach incidents accumulate more cash, suggesting that severely breached firms are those that face the need to build up more cash.

We examine the possible underlying mechanisms through which firms build up cash after a data breach incident. Breached firms can potentially build up their cash bal- 
ances by increasing their use of external debt and equity financing. However, given that breached firms are probably viewed as a higher credit risk by financial markets, we expect that they would find it difficult to raise external financing, and, therefore, external financing activities should decline after the disclosure of a data breach incident. Indeed, we find that both external equity and debt financing activities decline after a data breach incident. Given the lack of increase in external financing after a data breach incident, a test of investment as another possible mechanism naturally follows; that is, we test whether breached firms forgo investment in order to build up cash after a data breach incident. We find an economically important reduction in investment in both capital expenditure and acquisitions for firms exposed to data breaches, suggesting that firms reduce their investments to accumulate cash after a data breach incident.

Our paper makes several key contributions to the corporate finance literature. It is closely related to a vast literature that studies corporate cash holdings (see important contributions from Almeida et al. (2004); Arena and Julio (2015); Bates et al. (2009); Froot and Stein (1998); Han and Qiu (2007)). Our finding of data breach disclosure laws and actual data breaches affecting cash holdings is consistent with the precautionary cash holdings literature. However, the key contribution of our paper is the identification afforded by the staggered state-level laws and how firms can insulate themselves from the adverse effects of data breaches through liquidity management. Equally important, our paper provides evidence indicating that mandating the disclosure of data breaches amplifies the adverse consequences associated with future data breaches, with important implications for corporate liquidity policies. This result is consistent with that of Chen et al. (2020), who investigate how the quality of internal controls shapes the cash holding policies of Chinese firms.

The paper also contributes to the growing literature on data breach disclosure laws and actual data breaches. It complements the stream of research that examines the effects of data breach disclosure laws (Ashraf and Sunder (2018); Romanosky et al. (2011)). It also contributes to the stream of studies that examine the negative consequences of data 
breaches (Garg (2019); Gatzlaff and McCullough (2010); Kamiya et al. (2020); Lin et al. (2019); Mikhed and Vogan (2018); Rosati et al. (2017)).

This paper also adds new evidence to the law and finance literature. Specifically, our paper complements previous studies (e.g., Falato and Sim (2014); He (2018); Klasa et al. (2018); Qiu and Wang (2018)) that examine the impact of regulatory changes on corporate financial policies. While most of the explanations for corporate cash holding policies focus on firm-specific factors, recent studies show that firms change their financial policies in response to regulatory changes. For instance, He (2018) exploits reforms of state covenants-not-to-compete laws to capture exogenous changes in barriers for talent competition and finds that firms increase cash balances when the competition for talent intensifies. Falato and Sim (2014) exploit the staggered state-level changes in research and development $(\mathrm{R} \& \mathrm{D})$ tax credits to examine the impact of innovation on corporate cash holdings. They find that firms increase (decrease) cash balances in response to increase (cuts) in R\&D tax credits. We advance that data breaches represent a key operational risk that financial managers need to hedge against.

The remainder of the paper is structured as follows. Section 1.2 provides background information on the state-level disclosure laws. Section 1.3 describes the data and methodology. Sections 1.4 and 1.5 discuss the empirical results. Section 1.6 concludes the paper.

\section{$1.2 \quad$ Background}

\subsubsection{State-level data breach disclosure laws in the United States}

In the United States, the requirement to disclose data breaches is mainly governed by state laws. Prior to 2002, US firms were not obligated to disclose data breaches. Motivated by the data breach incident at the Stephen P. Teale Data Center, which affected the personally identifiable information of all 265,000 state employees, in 2002 California became the first state in the United States to enact a data breach disclosure law. The 
California data breach notification law was the first of its kind and has since served as the model for many other state-level disclosure laws.

As of March 29, 2018, all 50 US states, the District of Columbia, and the US territories of the Virgin Islands, Puerto Rico, and Guam had enacted mandatory disclosure laws requiring business and non-business entities to disclose and notify affected parties of data security breaches. ${ }^{4}$ Although details vary across states, the primary objective of all state-level data security breach disclosure laws is to obligate firms to publicly disclose data breaches involving non-public personally identifiable information, such as Social Security numbers (Romanosky et al. (2011)). The issue of disclosure is not limited to external threats to data security from cyber criminals but includes a firm's own internal compromise of security that leads to the accidental or unlawful destruction, loss, alteration, unauthorized disclosure of, or access to protected data transmitted, stored or otherwise processed. As explained earlier, the disclosure of a data breach could have direct and indirect adverse effect on corporations through remediation costs, regulatory action and fines, higher cost of capital, litigation, loss of customers and reduced revenue, loss of intellectual property and strategic knowledge. In the event of a data breach, a firm will have to investigate the source of the breach and take remedial actions to deal with the breach. Often times, the victims of data breaches have to spend considerably to overhaul their cyber security. Furthermore, regulatory agencies could impose fines when independent assessments show that the firm did not have adequate protective measures (such as encryption) in place. Victims of a disclosed data breach can seek legal remedy or compensation if it can be proven that the breached firm failed to properly protect personally identifiable information in accordance with its duties or had inadequate data security, and an actual harm such as identity theft has been suffered from the breach. Because firms only have to disclose a breach and not the protective measures it put in place, victims or plaintiffs (usually in a putative class action) mostly rely on cyberse-

\footnotetext{
${ }^{4}$ See the various state disclosure laws from the National Conference of State Legislatures, at http://www.ncsl.org/research/telecommunications-and-information-technology/securitybreach-notification-laws.aspx.
} 
curity and identity theft experts to determine the adequacy of a firm's level of security protection. Since the reasons for the changes in the state-level data breach disclosure laws were unrelated to the financing policies of firms, employing these legal reforms as exogenous shocks helps mitigate potential endogeneity concerns.

\subsubsection{Related literature}

Our work contributes to the growing literature on data breach disclosure laws and actual data breaches. A number of papers study the effects of mandatory data breach disclosure laws. For instance, Ashraf and Sunder (2018) examine the net effect of data breach disclosure laws on shareholder risk. They focus on explaining changes in the cost of equity following the passage of data breach laws and find that the cost of equity is significantly impacted by data breach laws, consistent with the idea that the increase in risk exposure prompts firms to react proactively to reduce exposure to data breach risks. Our paper complements this study by examining the link between mandatory data breach disclosure laws, data breaches, and corporate cash holdings.

Another stream of data breach research examines the negative consequences of data breaches. For instance, Lending et al. (2018) find that data breach firms have one-year buy-and-hold abnormal returns of $-3.5 \%$. Additionally, banks with breaches experience a significant decline in deposits, and non-banks experience significant declines in sales in the long run. Romanosky et al. (2014) empirically investigate the impact of data breaches on litigation risk and the outcomes of data breach litigation. The authors show that federal lawsuits due to data breaches result in a high settlement rate of about $50 \%$. They further show that the number of records compromised is positively related to the probability of being sued, and victims that claim financial harm from the data breach are more likely to sue the firm. Kamiya et al. (2020) report that successful data breaches are associated with significant declines in shareholder value, a deterioration in credit ratings, increased cash flow volatility, adversely affect sales growth, and decreased investments in the short run and result in an increase in the risk assessment of target firms. Gatzlaff 
and McCullough (2010) find that data breach announcements have a significant negative effect on shareholder wealth. Rosati et al. (2017) find that data breach announcements have a significant positive effect on the bid-ask spread and trading volumes. Lin et al. (2019) find statistically significant evidence of opportunistic insider trading in the months prior to data breach announcements. Garg (2019) finds that the effects of a data breach are not isolated to the breached firm, but spill over to peer firms. Mikhed and Vogan (2018) examine the interaction of consumers with the credit market and their use of credit following data breaches. The authors find that the victims of data breaches increase the acquisition of fraud protection services, are more likely to freeze their credit files, are likely to opt out of credit offers, but do not significantly switch lenders.

\subsection{Data and Methodology}

\subsubsection{Data Collection}

To examine the effects of the staggered state-level data breach disclosure laws on cash holdings, we collect initial firm-level data from the merged Center for Research in Security Prices (CRSP)/Compustat database for the period 1997-2015. This period covers the majority of the years in which the states passed data breach disclosure laws. Our sample period begins five years before California passed the first state-level data breach disclosure law, in 2002, and ends five years after Mississippi passed a similar law, in 2010. Following prior literature (Bates et al. (2009); Opler et al. (1999)), we exclude all financial firmsthat is, those with Standard Industrial Classification (SIC) codes 6000-6999- because their cash holdings include inventories of marketable securities and they are also required to meet statutory capital requirements. We exclude utility companies (SIC codes 49004999) because their cash holdings are possibly subject to regulatory supervision in some states. We further drop observations with negative or missing total book assets. This yields a final sample of 56,646 firm-year observations. 
Next, to examine the effect of actual data breaches on corporate cash holdings, we obtain data on data breaches from a chronological listing of disclosed data breaches available from the Privacy Rights Clearinghouse (PRC) for the period 2005-2018. ${ }^{5}$ PRC is a consumer advocacy not-for-profit organization that has been publishing all disclosed data breaches in the United States since 2005. The PRC data provides relevant disclosed data breach information. We collect information about the name of the breached firm, the breach disclosure date, a description of the breach incident, and, if available, the total number of records breached. We identify 329 nonfinancial business firms as having disclosed a data breach over the 2005-2018 sample period. We then manually merge the disclosed breaches with the CRSP/Compustat data.

\subsubsection{Estimation Technique}

The staggered timing of the passage of the state-level disclosure laws enables us to use states that had not yet passed disclosure laws at a given time (including states that eventually enacted disclosure laws, as well as states that never enacted them within the period of study), to control for potential confounding effects. Therefore, we use a difference-in-differences approach to empirically explore the effects of the disclosure laws on cash holdings. Our model setup mirrors that of Francis et al. (2014). We estimate

$$
\operatorname{Cash}_{i, s, t}=\alpha+\beta \text { Disclosure Law }(0 / 1)_{s, t}+\gamma X_{i, s, t}+\theta_{s}+\delta_{t}+\rho_{j}+\nu_{i}+\varepsilon_{i, s, t},
$$

where $i, s$, and $t$ index firm, state, and time, respectively. The dependent variable, Cash, is cash and marketable securities scaled by total book assets; Disclosure Law $(0 / 1)_{s, t}$ is a dummy variable that switches to one the year after the focal state passed the disclosure law; $X_{i, s, t}$ is a vector of controls; $\theta_{s}$ represents a set of state dummies that account for state-level unobservable factors that could be correlated with the data breach disclosure

\footnotetext{
${ }^{5}$ See https://www.privacyrights.org/.) PRC is the most cited source of information relating to data breaches (e.g., Kamiya et al. (2020); Romanosky et al. (2014)).
} 
laws, and thus bias our estimates; $\delta_{t}$ represents year dummies to control for secular shocks in cash holdings coinciding with the passage of the disclosure laws; and $\rho_{j}$ and $\nu_{i}$ capture industry and firm fixed effects, respectively. ${ }^{6}$ The term $\varepsilon_{i, s, t}$ is a random error term. We cluster standard errors by state, because the treatment is defined at the state level. Alternatively, we follow Falato and Sim (2014) and re-estimate the baseline difference-in-differences specification using first differences. As explained earlier, statelevel disclosure laws charge firms operating within the state (with data breach laws) with the responsibility of disclosing data breaches. Firms can operate in additional states besides their headquarters state and can thus be partly exposed to a data breach disclosure law prior to their home state passing a similar law. However, focusing on the states in which firms are headquartered is a conservative approach, since it essentially downward biases $\beta$ in Eq.(1.1), which should result in an underestimation of our treatment effect. In other words, firms that are partly pre-exposed to a data breach disclosure law will have a weaker reaction when a similar law is passed in their home state.

In addition, to examine the impact of data breaches on cash holdings, we follow Garg (2019) and estimate

$$
\operatorname{Cash}_{i, t}=\alpha+\beta \operatorname{Breach}(0 / 1)_{t}+\gamma X_{i, t}+\rho_{j}+\delta_{t}+\varepsilon_{i, t},
$$

where $\operatorname{Breach}(0 / 1)$ is a dummy variable set to one if a firm $i$ discloses a data breach in time $t$; and zero otherwise. All other variables maintain their previous definitions, and robust standard errors are estimated by clustering at the firm level.

\subsubsection{Variables}

We use the most traditional measure of cash in the literature (Bates et al. (2009); Opler et al. (1999)) as our dependent variable. We measure Cash as cash and marketable securities scaled by total book assets.

\footnotetext{
${ }^{6}$ The industry dummies are constructed based on the 49-industry classification of Fama and French (1997).
} 
As explained earlier, in addition to the dummies capturing disclosure laws, we construct variables to capture the impact of actual data breaches on cash holdings. The variable $\operatorname{Breach}(0 / 1)$ is a dummy that equals one for breached firms in the year of the data breach; and zero otherwise. We treat multiple breaches in a particular year as a single breach. The breaches are relatively evenly distributed over the sample period, reducing concerns that the data breaches are clustered in time.

We follow the literature (Bates et al. (2009); Opler et al. (1999)) in our empirical testing and control for several variables that affect firm cash policy. Specifically, we control for Firm Size, Firm Age, Book Leverage, Market-to-book, Cash Flow, Capital Expenditure, Acquisition Expenditure, Dividend Paying Firms (0/1), REDD Expenditure, Net Working Capital, and Industry Cash Flow Volatility. The definitions of all the variables are detailed in the Table 1.9. We winsorize all variables at the 1st and 99th percentiles to minimize the influence of outliers. Table 1.1 provides summary statistics of the key variables used in this study. We report the mean, standard deviation, 25th percentile, median, and 75th percentile.

\subsection{State-Level Disclosure Laws and Corporate Cash holdings}

In this section, we test how firms' cash holdings change with the passage of state-level data breach disclosure laws.

\subsubsection{Baseline Regression Results}

Table 1.2 presents the baseline estimation results. We include state, year, industry, and firm fixed effects in various specifications. Column (1) includes controls for year, industry, and state fixed effects. In Column (2), we include year and firm fixed effects. California is one of the largest and important state economies in the United States, where some of 
the world's largest corporations (e.g., Apple, Google, and Facebook) are headquartered. ${ }^{7}$ Therefore, in Column (3), we exclude California and re-estimate Eq.(1.1). Our aim is to isolate the effects of state-level data breach disclosure laws; therefore, in Column (4), we purposely exclude the financial crisis period (2007-2009) from the sample period. In Column (5), the standard errors are two-way clustered by state and year. Finally, in Column (6), we follow Falato and Sim (2014) and re-estimate our baseline regression using first differences. We thus control for firm fixed effects, as well as industry and state fixed effects.

Across all these specifications, the coefficient associated with Disclosure Law(0/1) is positive and statistically significant, which shows that, following the passage of state-level data breach disclosure laws, firms increased their cash holdings. We use the results in Column (1) of Table 1.2 to gauge the economic importance of state-level disclosure laws on corporate cash holdings. In Column (1), the coefficient associated with Disclosure $\operatorname{Law}(0 / 1)$ is 0.0076 . All else being equal, an increase in cash holdings by 0.0076 corresponds to a $3.8 \%$ increase from mean cash holdings $(0.2008)$ and $7.3 \%$ of median cash holdings (0.1044) for our sample firms.

A concern with the difference-in-differences design is that a change in firm cash holdings can precede a change in disclosure laws. We test for pre-existing trends by introducing a series of timing dummies in our baseline specification. The result from the timing effects estimation, which is reported in the Online Appendix for brevity, indicates that the baseline results are not driven by pre-existing trends. This finding implies that treated firms increase their cash holdings only after, and not prior to, changes in state-level data breach disclosure laws. $^{8}$

\footnotetext{
${ }^{7}$ Firms headquartered in California account for $18 \%$ of the observations in our sample.

${ }^{8}$ The Online Appendix is available at https://sites.google.com/site/mockeefe/Data.
} 


\subsubsection{Falsification Test}

Omitted variables coinciding with the timing of the passage of the state-level disclosure laws could drive the baseline results. If this is the case, then the changes in corporate cash holdings we attribute to state-level disclosure laws reflect mere association rather than causality. However, the disclosure laws were enacted at different times, providing multiple exogenous shocks, affecting the various states at different times. This enables us to overcome an obvious challenge facing single- shock studies, where the coincidence of a potential omitted variable and the single shock could affect the economic outcomes of interest. While the staggered nature of the enactment of the state-level disclosure laws makes it unlikely for an omitted variable to coincide with the disclosure laws, as explained earlier, we still address this concern by conducting a falsification test in a manner similar to that of Cornaggia et al. (2015).

We follow a two- step process. First, for each year, we randomly assign firms to the various states. Next, we randomly assign the states into the distribution of years when the various disclosure laws were passed. This helps maintain the actual distribution of years in which the various states passed the disclosure laws; however, it disrupts the correct assignment of the states to the years in which the laws were passed. Therefore, an unobservable shock that occurs at approximately the same time as the passage of

the state-level data breach disclosure laws would still reside within the baseline testing framework and, hence, drive the cash results. In contrast, if no such unobservable shocks exist, then we expect that the incorrect assignments of the firms and states to the disclosure law years should weaken our results for the re-estimated baseline specification. Thus, the falsely assumed disclosure law events should have no effect on cash holdings. Indeed, we do not find statistically significant effects of the state-level disclosure laws on corporate cash holdings following the random assignment. As reported in Table 1.3, the coefficients on the Disclosure Law(0/1) variable in Columns (1) to (4) are all statistically nonsignificant and, hence, not different from zero. The non-results in this test discount an omitted variable bias. 


\subsubsection{Role of Financial Constraints}

So far, our findings support the hypothesis that firms increase their cash holdings in response to the passage of data breach disclosure laws. We now examine the role of financial constraints in the effect of disclosure laws on corporate cash holdings. Specifically, following To et al. (2018) and Francis et al. (2014), we sort firms into financially constrained and unconstrained groups based on firm size, firm age, and dividend payout ratio. $^{9}$ For each year, we rank the firms over the sample period and categorize firms in the bottom terciles of the size, age, and dividend payout distributions as financially constrained. Note that the rankings are performed on an annual basis and this approach allows firms to migrate between groups in different years. We then create dummy variables for the three financial constraint measures. We create the dummy variables Small Firms(0/1), Young Firms(0/1), and Non-dividend Payer(0/1), which we set to one for firms in the bottom of the size, age, and dividend payout distributions, respectively, and zero otherwise. We interact the various financial constraint measures with the Disclosure $\operatorname{Law}(0 / 1)$ dummy to examine the role of financial constraints in the effects of disclosure laws on the cash policy of firms. In Table 1.4, we include year and firm fixed effects in all specifications. Table 1.4 presents the findings for the role of financial constraints. In Columns (1) to (3), the variable of interest is the interaction of the financial constraint measure with the disclosure law dummy. The results in Columns (1) to (3) indicate that the positive effect of disclosure laws on corporate cash holdings is driven by financially constrained firms.

\footnotetext{
${ }^{9}$ Farre-Mensa and Ljungqvist (2016) argue that known measures of financial constraints do not actually measure financial constraints, since constrained firms have no trouble raising debt. However, their definition of constraint has been criticized as being too lenient, since, according to their definition, every time a firm raises debt or equity, we could say they are unconstrained, even though these firms could be passing up many very promising projects.
} 


\subsection{Effects of Actual Data Breaches}

We now turn to the analysis of actual data beaches. We begin by first examining the association between data breaches and certain observable firm characteristics.

\subsubsection{Association between Data Breaches and Observable Firm Characteristics}

In this section, we examine the determinants of data breaches. Since past research provides no theoretical guidance regarding the determinants of data breaches, we consider the findings of this paper to be suggestive and exploratory, and not definitive determinants of data breaches. We report the results of the penalized maximum likelihood logistic regression in Table 1.5. The dependent variable, Breach(0/1), is a dummy variable that equals one for breached firms in the year of the data breach, and zero otherwise. The observable firm characteristics include Firm Size, Firm Age, Market-to-book, Book Leverage, Capital Expenditure, Acquisition Expenditure, Dividend Paying Firms (0/1), REDD Expenditure, Profitability, and Cash.

The results show that the coefficients of Firm Size and Firm Age are positive and statistically significant at less than the $1 \%$ level, suggesting that data breaches are more likely in large and visible firms. This finding reflects the view that larger and older firms could possess greater amounts of relevant records, data, or proprietary information that could be attractive to data intruders. Additionally, the results show that data breaches are more likely at financially constrained firms (captured by firm dividendpaying status), profitable firms (measured by their return on assets), R\&D- intensive firms, and firms with high growth potential. As Doyle et al. (2007) suggest, financially constrained firms might not have adequate resources (either money or time) to invest in effective data security systems, since that might not be their priority. In addition, the positive association between data breaches and growth opportunities suggests that growth can stress the effectiveness of a firm's internal control system; that is, a rapidly growing 
firm could outstrip its internal data security system and require more time, personnel, and processes to keep its internal data security systems up-to-date (Doyle et al. (2007)).

\subsubsection{Data Breaches and Cash Holdings}

In this section, we examine the impact of actual data breaches on corporate cash holdings. Table 1.6 presents the baseline estimation results. In Columns (1) and (2), we estimate Eq. (1.2) using contemporaneous and lagged breaches, respectively. Table 1.6 shows that the coefficient associated with Breach $(0 / 1)_{t}$ in Column (1) is positive and statistically significant at less than the $1 \%$ level, revealing a positive relationship between data breaches and corporate cash holdings. This result is economically important; given the Breach $(0 / 1)_{t}$ coefficient of 0.03 , all else being equal, an increase in cash holdings of 0.03 corresponds to a $13.7 \%$ increase from mean cash holdings (0.2185). In Column (2), the coefficient on Breach $(0 / 1)_{t-1}$ is positive and statistically significant at less than the $1 \%$ level. This result suggests breached firms continue to hold more cash in the year following the data breach.

While we find a positive relation between data breaches and cash holdings, one could still argue that the extra cash balance that was built up following the enactment of the disclosure laws should be used up by breached firms to meet the cash outlays associated with the breach incident. However, while firms build up their cash reserves after the enactment of the laws, it is possible that breached firms build up cash because of an unusually bad breach incident. Thus, the cash needs are plausibly unanticipated in the case of an unusually bad breach, and firms that are subject to such a breach increase their cash holdings.

We proxy for the severity of data breaches using the stock market's reaction to a breach announcement. We use an event study methodology to estimate the CARs around the data breach announcement. We calculate the CARs during the five-day event window around the disclosure of data breaches. We then sort the CARs into terciles and estimate three levels of data breach severity. The variable Severe Breach(0/1) proxies for the 
expected costs of an unusually bad breach, and it is a dummy set to one for a breach with CARs in the lowest tercile (highest mean negative returns), Moderate Breach(0/1) is set to one for a breach with CARs in the middle tercile, and Low Breach(0/1) is set to one for a breach with CARs in the highest tercile (least mean negative returns).

Columns (3) and (4) of Table 1.6 show the results of a test that relates the ex post cash buildup to the severity of the breach incident. The breach severity levels are contemporaneous in Column (3) but lagged in Column (4). The results in Column (3) show that the coefficient associated with Severe Breach $(0 / 1)_{t}$ is positive and statistically significant at less than the $5 \%$ level, revealing that firms with severe breach incidents accumulate more cash than firms with moderate- and low-severity breach incidents in the breach year. This

result is economically important; given a coefficient of 0.0348 for Severe Breach $(0 / 1)_{t}$, all else being equal, an increase in cash holdings by 0.0348 corresponds to a $15.9 \%$ increase from mean cash holdings (0.2185). In Column (4), one year after the breach, the results again show that firms with severe breaches accumulate more cash.

Finally, to address the concern that breached firms are inherently different from nonbreached firms, we implement the entropy balancing method of Hainmueller and $\mathrm{Xu}$ (2013). By using this matching procedure, we ensure that the treated firms (breached firms) are equivalent to the control firms (non-breached firms), which alleviates any concerns that differences in firm characteristics are influencing our results. The results for the entropy-balanced sample, available in the Online Appendix, confirm a positive relation between data breaches and corporate cash holdings.

\subsubsection{Mechanisms}

Our evidence so far suggests that firms increase their cash balances following exposure to a data breach incident. In this section, we explore possible underlying mechanisms through which firms build up cash. Specifically, we examine whether external financing and investment activities are possible underlying mechanisms through which breached firms accumulate cash. 


\section{Data Breaches and External Financing}

Firms that experience data breaches could build up their cash balances by increasing their use of external debt and equity financing. However, given that breached firms are probably viewed as a higher credit risk by the financial markets and therefore face higher costs of capital, we expect that they would find it difficult to raise external financing, and, therefore, external financing activities should decline after the disclosure of a breach incident. To test this, we specify the following model:

$$
\text { External Finance }{ }_{i, t}=\alpha+\beta \operatorname{Breach}(0 / 1)_{t}+\gamma X_{i, t}+\rho_{j}+\delta_{t}+\varepsilon_{i, t},
$$

Following Dierker et al. (2019), we use two measures of external financing: (1) External Equity Financing, estimated as the ratio of the difference between the sale of common and preferred stocks (SSTK) and the purchase of common and preferred stocks (PRSTKC) to total assets at the beginning of the year, and (2) External Debt Financing, estimated as the ratio of long-term debt issuance (DLTIS) minus long-term debt reduction (DLTR) to total assets at the beginning of the year. The term $X_{i, t}$ is a vector of controls. All other variables maintain their previous definitions, and robust standard errors are estimated by clustering at the firm level.

Table 1.7 reports the estimation results for the effects of data breaches on external financing activities. In Columns (1) and (3), we examine the effect of data breaches on external financing. The coefficients of Breach(0/1) for External Equity Financing and External Debt Financing are -0.014 and -0.0171, respectively, both statistically significant at the $1 \%$ level, as well as economically important. Given Breach(0/1) coefficients of -0.014 and -0.0171, respectively, for External Equity Financing and External Debt Financing, all else being equal, a decrease in External Equity Financing by 0.014 corresponds to a $32 \%$ decrease from mean External Equity Financing (0.0436), and a decrease in External Debt Financing by 0.0171 corresponds to a $62 \%$ decrease from mean External Debt Financing (0.0275). In Columns (2) and (4), we examine the impact of data breach severity on the 
external financing activities of firms. Overall, the results show that external financing declines following a data breach incident, and firms are even less likely to raise external finance when they experience an unusually severe breach incident.

\section{Data Breaches and Corporate Investment}

Given the lack of increase in external financing after a data breach incident, a natural follow-up mechanism that requires testing is whether breached firms forgo investment to build up cash after a data breach incident. That is, do firms forgo investment to accumulate cash after a data breach incident? To examine this possibility, we follow Arena and Julio (2015) and estimate the investment regression

$$
\text { Investment }_{i, t}=\alpha+\beta \operatorname{Breach}(0 / 1)_{t}+\gamma X_{i, t}+\rho_{j}+\delta_{t}+\varepsilon_{i, t}
$$

where Investment $t_{i, t}$ is investment in either Capital Expenditure or Acquisition Expenditure, with Capital Expenditure measured as the ratio of capital expenditure to total book assets at the beginning of the year and Acquisition Expenditure measured as the ratio of acquisitions to total book assets at the beginning of the year. The term $X_{i, t}$ is a vector of controls. All the other variables maintain their previous definitions, and robust standard errors are estimated by clustering at the firm level.

Columns (1) and (2) of Table 1.8 report the results for investment in Capital Expenditure, and Columns (3) and (4) present the results for investment in Acquisition Expenditure. In Columns (1) and (3), we examine, respectively, the effect of data breaches on capital expenditure and acquisition expenditure. The Breach(0/1) coefficients for Capital Expenditure and Acquisition Expenditure are -0.0072 and -0.0209, respectively. All else being equal, a decrease in Capital Expenditure by 0.0072 corresponds to an $11.8 \%$ decrease from mean Capital Expenditure (0.061), and a decrease in Acquisition Expenditure by 0.0209 corresponds to a $52 \%$ decrease from mean Acquisition Expenditure (0.0399). In Columns 2 and 4, we examine the impact of data breach severity on capital expenditure and acquisition expenditure, respectively. Overall, the results in Table 1.8 show that 
investment in both capital and acquisition expenditures decline following a data breach incident, especially when the breach is severe. This result is consistent with the view that firms forgo investment in capital expenditure and acquisitions to accumulate cash.

\subsection{Conclusion}

Prior to 2002, US firms were not obligated to disclose data breaches. From 2002 to 2016, state-level disclosure laws mandating firms to disclose data breaches were passed in almost all the US states. These disclosure laws impose "disclosure costs" and inadvertently compel firms to internalize the costs (including negative externalities, such as identity theft) of their data insecurity. We argue that, holding constant the underlying likelihood of experiencing a data breach, mandatory data breach disclosure laws increase the cash flow risk associated with future data breaches, and firms build up balance sheet liquidity as a precautionary measure. We provide such evidence by studying the effects of data breach disclosure laws and actual data breaches on the cash holding policies of US public firms.

Exploiting a series of natural experiments regarding staggered state-level data breach disclosure laws, we show that the passage of mandatory data breach disclosure laws leads to an increase in corporate cash holdings, particularly among financially constrained firms. Although we cannot entirely rule out the possibility that an omitted variable affected by data breach disclosure laws is driving firm cash holdings, the staggered nature of the state-level laws and the evidence from a falsification test make such a possibility unlikely. The finding is also robust to a dynamic effect estimation that addresses the parallel trends assumption. Additionally, we probe the impact of actual data breaches on corporate cash holdings and find that firms increase their cash balances following a data breach, as well as decrease their external financing and investment activities. 


\section{Bibliography}

Acharya, V., Davydenko, S. A., and Strebulaev, I. A. (2012). Cash holdings and credit risk. The Review of Financial Studies, 25(12):3572-3609.

Almeida, H., Campello, M., and Weisbach, M. S. (2004). The cash flow sensitivity of cash. The Journal of Finance, 59(4):1777-1804.

Arena, M. and Julio, B. (2015). The effects of securities class action litigation on corporate liquidity and investment policy. Journal of Financial and Quantitative Analysis, 50(1):251-275.

Ashraf, M. and Sunder, J. (2018). Mandatory disclosure of cyber incidents and the cost of equity. SSRN.

Bates, T. W., Kahle, K. M., and Stulz, R. M. (2009). Why do US firms hold so much more cash than they used to? The Journal of Finance, 64(5):1985-2021.

Beck, T., Levine, R., and Levkov, A. (2010). Big bad banks? the winners and losers from bank deregulation in the united states. The Journal of Finance, 65(5):1637-1667.

Brav, A., Graham, J. R., Harvey, C. R., and Michaely, R. (2005). Payout policy in the 21st century. Journal of Financial Economics, 77(3):483-527.

Chen, H., Yang, D., Zhang, J. H., and Zhou, H. (2020). Internal controls, risk management, and cash holdings. Journal of Corporate Finance, 64(1):1-20.

Cornaggia, J., Mao, Y., Tian, X., and Wolfe, B. (2015). Does banking competition affect innovation? Journal of Financial Economics, 115(1):189-209.

Deloitte. (2018). Using a Loss Reserve to Manage Cyber Risk in the Federal Sector. Available at https://deloitte.wsj.com/articles/using-a-loss-reserve-to-manage-cyber-risk-inthe-federal-sector-1532408112.

Dierker, M., Lee, I., and Seo, S. W. (2019). Risk changes and external financing activities: Tests of the dynamic trade-off theory of capital structure. Journal of Empirical Finance, 52:178-200.

Doyle, J., Ge, W., and McVay, S. (2007). Determinants of weaknesses in internal control over financial reporting. Journal of Accounting and Economics, 44(1):193-223.

Falato, A. and Sim, J. (2014). Why do innovative firms hold so much cash? evidence from changes in state R\&D tax credits. SSRN. 
Fama, E. F. and French, K. R. (1997). Industry costs of equity. Journal of Financial Economics, 43(2):153-193.

Farre-Mensa, J. and Ljungqvist, A. (2016). Do measures of financial constraints measure financial constraints? The Review of Financial Studies, 29(2):271-308.

Francis, B., Hasan, I., and Wang, H. (2014). Banking deregulation, consolidation, and corporate cash holdings: US evidence. Journal of Banking Ef Finance, 41:45-56.

Froot, K. A. and Stein, J. C. (1998). Risk management, capital budgeting, and capital structure policy for financial institutions: an integrated approach. Journal of Financial Economics, 47(1):55-82.

Fuhrmans, V. (2017). A new worry for CEOs: A career-ending cyberattack. The Wall Street Journal.

Garg, P. (2019). Cybersecurity breaches and cash holdings: Spillover effect. Financial Management, 49(2):503-519.

Gatzlaff, K. M. and McCullough, K. A. (2010). The effect of data breaches on shareholder wealth. Risk Management and Insurance Review, 13(1):61-83.

Hainmueller, J. and $\mathrm{Xu}, \mathrm{Y}$. (2013). Ebalance: A Stata package for entropy balancing. Journal of Statistical Software, 54(7):1-18.

Han, S. and Qiu, J. (2007). Corporate precautionary cash holdings. Journal of Corporate Finance, 13(1):43-57.

He, Z. (2018). Money held for moving stars: Talent competition and corporate cash holdings. Journal of Corprate Finance, 51:210-234.

Kamiya, S., Kang, J.-K., Kim, J., Milidonis, A., and Stulz, R. M. (2020). Risk management, firm reputation, and the impact of successful cyberattacks on target firms. Journal of Financial Economics.

Klasa, S., Ortiz-Molina, H., Serfling, M., and Srinivasan, S. (2018). Protection of trade secrets and capital structure decisions. Journal of Financial Economics, 128(2):266286.

Lending, C., Minnick, K., and Schorno, P. J. (2018). Corporate governance, social responsibility, and data breaches. Financial Review, 53(2):413-455.

Lin, Z., Sapp, T., Ulmer, J. R., and Parsa, R. (2019). Insider trading ahead of cyber breach announcements. Journal of Financial Markets, 50:1-15.

Mikhed, V. and Vogan, M. (2018). How data breaches affect consumer credit. Journal of Banking and Finance, 88(1):192-207.

Opler, T., Pinkowitz, L., Stulz, R., and Williamson, R. (1999). The determinants and implications of corporate cash holdings. Journal of Financial Economics, 52(1):3-46. 
Ponemon Institute. (2018). 2018 Cost of Data Breach Study: Impact of Business Continuity Management. Available at https://www.ibm.com/downloads/cas/AEJYBPWA.

Qiu, B. and Wang, T. (2018). Does knowledge protection benefit shareholders? evidence from stock market reaction and firm investment in knowledge assets. Journal of Financial and Quantitative Analysis, 53(3):1341-1370.

Riddick, L. A. and Whited, T. M. (2009). The corporate propensity to save. The Journal of Finance, 64(4):1729-1766.

Romanosky, S., Hoffman, D., and Acquisti, A. (2014). Empirical analysis of data breach litigation. Journal of Empirical Legal Studies, 11(1):74-104.

Romanosky, S., Telang, R., and Acquisti, A. (2011). Do data breach disclosure laws reduce identity theft? Journal of Policy Analysis and Management, 30(2):256-286.

Rosati, P., Cummins, M., Deeney, P., Gogolin, F., Werff, L., and Lynn, T. (2017). The effect of data breach announcements beyond the stock price: Empirical evidence on market activity. International Review of Financial Analysis, 49(1):146-154.

To, T., Navone, M., and Wu, E. (2018). Analyst coverage and the quality of corporate investment decisions. Journal of Corporate Finance, 51:164-181.

Ucar, E. (2019). Creative culture, risk-taking, and corporate financial decisions. European Financial Management, 25(3):684-717. 
Table 1.1: Summary statistics

This table reports the summary statistics of the key variables. Panel A reports the summary statistics for the state-level disclosure law sample over the period 1997 to 2015. Panel B covers the data breach subsample, where the sample period is from 2011 to 2016. See Table 1.9 for the variable definitions.

\begin{tabular}{|c|c|c|c|c|c|c|}
\hline \multicolumn{7}{|c|}{ Panel A: Disclosure law sample (1997-2015) } \\
\hline Variables & Obs & Mean & $\mathrm{SD}$ & 25 th & Median & 75 th \\
\hline Cash & 56,646 & 0.2008 & 0.2231 & 0.0285 & 0.1044 & 0.2913 \\
\hline Firm Size & 56,646 & 5.6136 & 2.0593 & 4.0819 & 5.5717 & 7.0455 \\
\hline Firm Age & 56,646 & 7.3003 & 0.8889 & 2.0179 & 5.0435 & 11.0918 \\
\hline Market-to-book & 56,646 & 2.1592 & 2.9919 & 1.1104 & 1.5113 & 2.3119 \\
\hline Cash Flow & 56,646 & -0.3758 & 4.8386 & -0.0150 & 0.0684 & 0.1210 \\
\hline Book Leverage & 56,646 & 0.2362 & 0.3177 & 0.0229 & 0.1863 & 0.3528 \\
\hline Capital Expenditure & 56,646 & 0.0657 & 0.1248 & 0.0174 & 0.0364 & 0.0729 \\
\hline Acquisition Expenditure & 56,646 & 0.0451 & 0.1672 & 0 & 0 & 0.0144 \\
\hline RED Expenditure & 56,646 & 0.0709 & 0.1895 & 0 & 0.0021 & 0.0738 \\
\hline Industry Cash Flow Volatility & 56,646 & 0.3440 & 0.5270 & 0.0825 & 0.1403 & 0.2499 \\
\hline Net Working Capital & 56,646 & -0.0584 & 1.0372 & -0.0589 & 0.0636 & 0.2157 \\
\hline Dividend Paying Firms (0/1) & 56,646 & 0.2767 & 0.4473 & 0 & 0 & 1 \\
\hline \multicolumn{7}{|c|}{ Panel B: Data breach subsample (2005-2018) } \\
\hline $\operatorname{Breach}(0 / 1)$ & 42,893 & 0.0067 & 0.0776 & 0 & 0 & 0 \\
\hline Cash & 42,893 & 0.2185 & 0.2398 & 0.0426 & 0.1251 & 0.3062 \\
\hline Firm Size & 42,893 & 6.5181 & 2.1207 & 4.7692 & 6.3149 & 7.8124 \\
\hline Firm Age & 42,893 & 7.3113 & 0.7520 & 1.8000 & 5.6335 & 10.1718 \\
\hline Market-to-book & 42,893 & 2.0807 & 3.2412 & 0.5860 & 1.0410 & 1.9100 \\
\hline Cash Flow & 42,893 & -0.5429 & 5.9620 & -0.0121 & 0.0716 & 0.1194 \\
\hline Book Leverage & 42,893 & 0.2356 & 0.3177 & 0.0180 & 0.1895 & 0.3468 \\
\hline Capital Expenditure & 42,893 & 0.0610 & 0.3175 & 0.0145 & 0.0322 & 0.0658 \\
\hline Acquisition Expenditure & 42,893 & 0.0399 & 0.7749 & 0 & 0 & 0.0127 \\
\hline External Equity Financing & 42,893 & 0.0436 & 0.2193 & -0.0093 & 0 & 0.0077 \\
\hline External Debt Financing & 42,893 & 0.0275 & 0.1297 & -0.0129 & 0 & 0.0226 \\
\hline RED Expenditure & 42,893 & 0.0725 & 0.2369 & 0 & 0.0036 & 0.0674 \\
\hline Industry Cash Flow Volatility & 42,893 & 0.4858 & 0.8114 & 0.0818 & 0.1507 & 0.2696 \\
\hline Net Working Capital & 42,893 & -0.1650 & 9.7913 & -0.0793 & 0.0224 & 0.1637 \\
\hline Dividend Paying Firms (0/1) & 42,893 & 0.3472 & 0.4761 & 0 & 0 & 1 \\
\hline
\end{tabular}


Table 1.2: State-level disclosure laws and cash holdings

This table reports the estimation results of Eq. (1.1), which tests whether firms change their cash holdings in response to changes in state-level data breach disclosure laws. The dependent variable is Cash and the functional form is linear. The variable Disclosure $\operatorname{Law}(0 / 1)$ is a dummy that switches to one the year after the focal state passes the disclosure law.

The definitions of the control variables are presented in Table 1.9. In Columns (1) to (4), standard errors are clustered by state, but, in Column (5), the standard errors are two-way clustered by state and year. The specification in Column (6) is estimated using ordinary least squares in first differences to remove firm fixed effects in the levels equation and to control for year, industry, and state fixed effects. Heteroskedasticity-consistent standard errors are reported in parentheses. $* * *$, $* *$, and $*$ denote significance at the $1 \%, 5 \%$, and $10 \%$ levels, respectively.

\begin{tabular}{|c|c|c|c|c|c|c|}
\hline & \multicolumn{6}{|c|}{ Dependent Variable: Cash } \\
\hline & $\begin{array}{l}\text { Baseline } \\
\text { (1) }\end{array}$ & $\begin{array}{c}\text { Bas. w/ FE } \\
(2)\end{array}$ & $\begin{array}{c}\text { Bas. exc. CA } \\
(3)\end{array}$ & Bas. exc. GFC & State \& Year SE & $\begin{array}{c}\text { First-diff Est } \\
(6)\end{array}$ \\
\hline Disclosure Law(0/1) & $\begin{array}{l}0.0076^{* *} \\
(0.0031)\end{array}$ & $\begin{array}{c}0.0056^{* *} \\
(0.0027)\end{array}$ & $\begin{array}{c}0.0032^{* *} \\
(0.0012)\end{array}$ & $\begin{array}{r}0.0078^{* *} \\
(0.0038)\end{array}$ & $\begin{array}{c}0.0076^{* * *} \\
(0.0028)\end{array}$ & $\begin{array}{c}0.0026^{* *} \\
(0.0015)\end{array}$ \\
\hline Firm Size & $\begin{array}{c}-0.0110 * * * \\
(0.0022)\end{array}$ & $\begin{array}{c}-0.0094^{* * *} \\
(0.0019)\end{array}$ & $\begin{array}{c}-0.0132^{* * *} \\
(0.0014)\end{array}$ & $\begin{array}{c}-0.0100 * * * \\
(0.0021)\end{array}$ & $\begin{array}{c}-0.0110 * * * \\
(0.0009)\end{array}$ & $\begin{array}{c}0.0179 * * * \\
(0.0046)\end{array}$ \\
\hline Market-to-book & $\begin{array}{c}0.0080 * * * \\
(0.0015)\end{array}$ & $\begin{array}{c}0.0023^{* * * *} \\
(0.0008)\end{array}$ & $\begin{array}{c}0.0079^{* * *} \\
(0.0019)\end{array}$ & $\begin{array}{c}0.0071^{* * *} * \\
(0.0014)\end{array}$ & $\begin{array}{c}0.0080^{* * *} \\
(0.0018)\end{array}$ & $\begin{array}{l}0.0005 \\
(0.0004)\end{array}$ \\
\hline Firm Age & $\begin{array}{c}-0.0213^{* * *} \\
(0.0044)\end{array}$ & $\begin{array}{c}-0.0320 * * * \\
(0.0051)\end{array}$ & $\begin{array}{c}-0.0171^{* * *} \\
(0.0028)\end{array}$ & $\begin{array}{c}-0.0217^{* * *} \\
(0.0045)\end{array}$ & $\begin{array}{c}-0.0213^{* * *} \\
(0.0014)\end{array}$ & $\begin{array}{l}-0.0031 \\
(0.0027)\end{array}$ \\
\hline Book Leverage & $\begin{array}{c}-0.1400 * * * \\
(0.0206)\end{array}$ & $\begin{array}{c}-0.0851^{* * *} \\
(0.0116)\end{array}$ & $\begin{array}{c}-0.1283^{* * *} \\
(0.0185)\end{array}$ & $\begin{array}{c}-0.1711^{* * *} \\
(0.0210)\end{array}$ & $\begin{array}{c}-0.1400 * * * \\
(0.0150)\end{array}$ & $\begin{array}{c}-0.0484^{* * *} \\
(0.0098)\end{array}$ \\
\hline Cash Flow & $\begin{array}{c}-0.0049 * * * \\
(0.0018)\end{array}$ & $\begin{array}{l}-0.0005 \\
(0.0006)\end{array}$ & $\begin{array}{c}-0.0044^{* *} \\
(0.0018)\end{array}$ & $\begin{array}{c}-0.0004 \\
(0.0016)\end{array}$ & $\begin{array}{c}-0.0049 * * * \\
(0.0012)\end{array}$ & $\begin{array}{l}-0.0003 \\
(0.0003)\end{array}$ \\
\hline Capital Expenditure & $\begin{array}{c}-0.0709 * * \\
(0.0280)\end{array}$ & $\begin{array}{l}-0.0221 \\
(0.0135)\end{array}$ & $\begin{array}{c}-0.0537^{* * *} \\
(0.0181)\end{array}$ & $\begin{array}{c}-0.0666 * * \\
(0.0285)\end{array}$ & $\begin{array}{c}-0.0709 * * * \\
(0.0206)\end{array}$ & $\begin{array}{c}-0.0191^{* * *} \\
(0.0066)\end{array}$ \\
\hline Acquisition Expenditure & $\begin{array}{l}-0.0052 \\
(0.0048)\end{array}$ & $\begin{array}{l}-0.0023 \\
(0.0023)\end{array}$ & $\begin{array}{l}-0.0041 \\
(0.0037)\end{array}$ & $\begin{array}{l}-0.0045 \\
(0.0041)\end{array}$ & $\begin{array}{l}-0.0052 \\
(0.0050)\end{array}$ & $\begin{array}{l}-0.0038 \\
(0.0038)\end{array}$ \\
\hline Dividend Paying Firms (0/1) & $\begin{array}{c}-0.0072^{* *} \\
(0.0028)\end{array}$ & $\begin{array}{c}0.0094^{* * *} \\
(0.0033)\end{array}$ & $\begin{array}{c}-0.0066^{* *} \\
(0.0031)\end{array}$ & $\begin{array}{c}-0.0087^{* * *} * \\
(0.0028)\end{array}$ & $\begin{array}{c}-0.0072^{* * *} \\
(0.0021)\end{array}$ & $\begin{array}{c}0.0027 \\
(0.0017)\end{array}$ \\
\hline RED Expenditure & $\begin{array}{c}0.1909 * * * \\
(0.0281)\end{array}$ & $\begin{array}{c}0.0204^{* * *} \\
(0.0072)\end{array}$ & $\begin{array}{c}0.1726^{* * *} \\
(0.0262)\end{array}$ & $\begin{array}{c}0.1790 * * * \\
(0.0288)\end{array}$ & $\begin{array}{c}0.1909^{* * *} \\
(0.0220)\end{array}$ & $\begin{array}{c}0.0194^{* * *} \\
(0.0042)\end{array}$ \\
\hline Net Working Capital & $\begin{array}{l}-0.0001 \\
(0.0004)\end{array}$ & $\begin{array}{c}-0.0162^{* * *} \\
(0.0022)\end{array}$ & $\begin{array}{l}-0.0001 \\
(0.0004)\end{array}$ & $\begin{array}{c}-0.0344^{* * *} \\
(0.0039)\end{array}$ & $\begin{array}{l}-0.0001 \\
(0.0005)\end{array}$ & $\begin{array}{c}-0.0116^{* * *} \\
(0.0014)\end{array}$ \\
\hline Industry Cash Flow Volatility & $\begin{array}{c}0.0273^{* * *} \\
(0.0068)\end{array}$ & $\begin{array}{l}-0.0031 \\
(0.0064)\end{array}$ & $\begin{array}{c}0.0207^{* * *} \\
(0.0056)\end{array}$ & $\begin{array}{c}0.0238 * * * \\
(0.0067)\end{array}$ & $\begin{array}{c}0.0273^{* * *} \\
(0.0056)\end{array}$ & $\begin{array}{c}0.0005 \\
(0.0046)\end{array}$ \\
\hline Year Fixed Effects & Yes & Yes & Yes & Yes & Yes & Yes \\
\hline Industry Fixed Effects & Yes & No & Yes & Yes & Yes & Yes \\
\hline State Fixed Effects & Yes & No & Yes & Yes & Yes & Yes \\
\hline Observations & 56,646 & 56,646 & 47,526 & 48,551 & 56,646 & 47,117 \\
\hline$A d j . R^{2}$ & 0.4939 & 0.0691 & 0.4658 & 0.5083 & 0.4287 & 0.1291 \\
\hline
\end{tabular}


Table 1.3: Randomization of state-level data breach disclosure laws

This table reports the estimation results of Eq. (1.1) with randomized disclosure laws. We follow a two-step process in the randomization test. First, for each year, we randomly assign firms to the various states. Next, we randomly assign the states within the distribution of years in which the various disclosure laws were passed. We thus maintain the actual distribution of years in which the various states passed the disclosure laws; however, this approach disrupts the correct assignment of the states to the years in which the laws were passed. The dependent variable in all the columns is Cash, measured as cash and marketable securities scaled by total book assets at the beginning of the year. Standard errors clustered by state are reported in parentheses, with $1 \%, 5 \%$, and $10 \%$ levels of statistical significance denoted by $* * *, * *$, and $*$, respectively.

\begin{tabular}{lcccc}
\hline \hline & & Dependent & Variable: Cash \\
Variables & $(1)$ & $(2)$ & $(3)$ & $(4)$ \\
\hline Disclosure Law(0/1) & 0.0008 & 0.0008 & 0.0007 & 0.0008 \\
& $(0.0024)$ & $(0.0023)$ & $(0.0023)$ & $(0.0022)$ \\
Firm Size & $-0.0104^{* * *}$ & $-0.0109^{* * *}$ & $-0.0110^{* * *}$ & $-0.0095^{* * *}$ \\
& $(0.0022)$ & $(0.0027)$ & $(0.0022)$ & $(0.0020)$ \\
Market-to-book & $0.0083^{* * *}$ & $0.0094^{* * *}$ & $0.0079^{* * *}$ & $0.0023^{* * *}$ \\
& $(0.0016)$ & $(0.0018)$ & $(0.0015)$ & $(0.0008)$ \\
Firm Age & $-0.0232^{* * *}$ & $-0.0241^{* * *}$ & $-0.0212^{* * *}$ & $-0.0319^{* * *}$ \\
& $(0.0050)$ & $(0.0039)$ & $(0.0044)$ & $(0.0051)$ \\
Book Leverage & $-0.1464^{* * *}$ & $-0.1531^{* * *}$ & $-0.1399^{* * *}$ & $-0.0849^{* * *}$ \\
& $(0.0220)$ & $(0.0221)$ & $(0.0207)$ & $(0.0116)$ \\
Cash Flow & $-0.0048^{* *}$ & $-0.0052^{* *}$ & $-0.0049^{* * *}$ & -0.0005 \\
Capital Expenditure & $(0.0019)$ & $(0.0020)$ & $(0.0018)$ & $(0.0006)$ \\
& $-0.0747^{* *}$ & $-0.1026^{* * *}$ & $-0.0706^{* *}$ & -0.0218 \\
Acquisition Expenditure & $(0.0293)$ & $(0.0366)$ & $(0.0280)$ & $(0.0134)$ \\
& -0.0055 & -0.0054 & -0.0052 & -0.0023 \\
Dividend Paying Firms $(0 / 1)$ & $(0.0050)$ & $(0.0049)$ & $(0.0048)$ & $(0.0023)$ \\
& $-0.0139^{* * *}$ & $-0.0185^{* * *}$ & $-0.0073^{* *}$ & $0.0092^{* * *}$ \\
R\&BD Expenditure to assets & $(0.0035)$ & $(0.0048)$ & $(0.0028)$ & $(0.0033)$ \\
& $0.2076^{* * *}$ & $0.2838^{* * *}$ & $0.1909^{* * *}$ & $0.0202^{* * *}$ \\
Net Working Capital & $(0.0307)$ & $(0.0377)$ & $(0.0281)$ & $(0.0072)$ \\
Industry Cash Flow Volatility & $0.0273^{* * *}$ & $0.0908^{* * *}$ & $0.0272^{* * *}$ & -0.0031 \\
Year Fixed Effects & $(0.0068)$ & $(0.0071)$ & $(0.0068)$ & $(0.0064)$ \\
Industry Fixed Effects & Yes & Yes & Yes & Yes \\
State Fixed Effects & Yes & No & Yes & No \\
Firm Fixed Effects & No & Yes & Yes & No \\
Observations & No & No & No & Yes \\
Adj. R ${ }^{2}$ & 56,272 & 56,272 & 56,272 & 56,272 \\
\hline & 0.4613 & 0.4446 & 0.4938 & 0.0688 \\
\hline & & & &
\end{tabular}


Table 1.4: Disclosure laws and corporate cash holdings: Role of financial constraints

This table reports the role of financial constraints in the effect oof data breach disclosure laws on cash holdings. For each year, we rank the firms over the sample period and categorize those in the bottom terciles of the size, age, and dividend payout distributions as financially constrained. We then create dummy variables for the three financial constraint measures. We create the dummy variables Small Firms(0/1), Young Firms(0/1), and Non-dividend Payer(0/1) and set them to equal one for firms in the bottom of the size, age, and dividend payout distributions, and zero otherwise. Standard errors clustered by state are reported in parentheses, with $1 \%, 5 \%$, and $10 \%$ levels of statistical significance denoted by ***,**, and ${ }^{*}$, respectively.

\begin{tabular}{|c|c|c|c|}
\hline \multirow[b]{2}{*}{ Variables } & \multicolumn{3}{|c|}{ Dependent Variable: Cash } \\
\hline & (1) & $(2)$ & (3) \\
\hline Disclosure Law(0/1) & $\begin{array}{l}-0.0219 \\
(0.0162)\end{array}$ & $\begin{array}{l}-0.0052 \\
(0.0099)\end{array}$ & $\begin{array}{c}0.0276^{* * *} \\
(0.0089)\end{array}$ \\
\hline Small Firms $(0 / 1) \times$ Disclosure Law(0/1) & $\begin{array}{c}0.0344^{* *} \\
(0.0153)\end{array}$ & & \\
\hline Young Firms $(0 / 1) \times$ Disclosure Law(0/1) & & $\begin{array}{l}0.0216^{*} \\
(0.0128)\end{array}$ & \\
\hline Non-dividend Payer $(0 / 1) \times$ Disclosure Law $(0 / 1)$ & & & $\begin{array}{c}0.0369^{* * *} \\
(0.0085)\end{array}$ \\
\hline Firm Size & $\begin{array}{c}0.0901 * * * \\
(0.0170)\end{array}$ & $\begin{array}{c}0.0872^{* * *} \\
(0.0159)\end{array}$ & $\begin{array}{c}0.0871^{* * *} \\
(0.0159)\end{array}$ \\
\hline Market-to-book & $\begin{array}{l}-0.0018 \\
(0.0040)\end{array}$ & $\begin{array}{l}-0.0018 \\
(0.0040)\end{array}$ & $\begin{array}{l}-0.0018 \\
(0.0040)\end{array}$ \\
\hline Firm Age & $\begin{array}{c}-0.0444^{* * *} \\
(0.0156)\end{array}$ & $\begin{array}{c}-0.0440^{* * *} \\
(0.0147)\end{array}$ & $\begin{array}{c}-0.0371^{* *} \\
(0.0152)\end{array}$ \\
\hline Book Leverage & $\begin{array}{c}-0.1634^{* * *} \\
(0.0305)\end{array}$ & $\begin{array}{c}-0.1644^{* * *} \\
(0.0305)\end{array}$ & $\begin{array}{c}-0.1644^{* * *} \\
(0.0307)\end{array}$ \\
\hline Cash Flow & $\begin{array}{c}0.0022 \\
(0.0021)\end{array}$ & $\begin{array}{c}0.0022 \\
(0.0021)\end{array}$ & $\begin{array}{c}0.0022 \\
(0.0021)\end{array}$ \\
\hline Capital Expenditure & $\begin{array}{l}1.2590^{*} \\
(0.6937)\end{array}$ & $\begin{array}{l}1.2606^{*} \\
(0.6937)\end{array}$ & $\begin{array}{l}1.2594^{*} \\
(0.6937)\end{array}$ \\
\hline Acquisition Expenditure & $\begin{array}{c}-0.0613^{* *} \\
(0.0299)\end{array}$ & $\begin{array}{c}-0.0601^{* *} \\
(0.0298)\end{array}$ & $\begin{array}{c}-0.0597^{* *} \\
(0.0297)\end{array}$ \\
\hline Dividend Paying Firms (0/1) & $\begin{array}{c}-0.0159^{* *} \\
(0.0073)\end{array}$ & $\begin{array}{c}-0.0166^{* *} \\
(0.0071)\end{array}$ & $\begin{array}{c}-0.0326^{* * *} \\
(0.0076)\end{array}$ \\
\hline RED Expenditure & $\begin{array}{c}1.1079^{* * *} \\
(0.2035)\end{array}$ & $\begin{array}{c}1.1076^{* * *} \\
(0.2034)\end{array}$ & $\begin{array}{c}1.1076^{* * *} \\
(0.2033)\end{array}$ \\
\hline Net Working Capital & $\begin{array}{c}-0.0403^{*} \\
(0.0203)\end{array}$ & $\begin{array}{c}-0.0403^{*} \\
(0.0203)\end{array}$ & $\begin{array}{c}-0.0403^{*} \\
(0.0203)\end{array}$ \\
\hline Industry Cash Flow Volatility & $\begin{array}{l}-0.0003 \\
(0.0227)\end{array}$ & $\begin{array}{c}0.0035 \\
(0.0235)\end{array}$ & $\begin{array}{c}0.0067 \\
(0.0228)\end{array}$ \\
\hline Year Fixed Effects & Yes & Yes & Yes \\
\hline Firm Fixed Effects & Yes & Yes & Yes \\
\hline Observations & 56,645 & 56,645 & 56,645 \\
\hline Adj. $R^{2}$ & 0.1528 & 0.1526 & 0.1528 \\
\hline
\end{tabular}


Table 1.5: Association between data breaches and observable firm characteristics

This table presents the results for the association between data breaches and firm characteristics, using a penalized maximum likelihood logistic regression. The dependent variable is Breach(0/1). Column (1) is estimated with no industry fixed effects, but Column (2) includes industry fixed effects. All the variables are defined in Table 1.9. The sample period is from 2005 through 2018. The 1\%, 5\%, $10 \%$ levels of statistical significance are denoted by $* * *, * *$, and $*$, respectively.

\begin{tabular}{lcc}
\hline \hline & Dependent Variable: Breach(0/1) \\
Variables & $(1)$ & $(2)$ \\
\hline Firm Size & $0.6779^{* * *}$ & $0.7089^{* * *}$ \\
Firm Age & $(0.0442)$ & $(0.0471)$ \\
& $0.3667^{* * *}$ & $0.4313^{* * *}$ \\
Market-to-book & $(0.0821)$ & $(0.0858)$ \\
& $0.0031^{* * *}$ & $0.0033^{* * *}$ \\
Book Leverage & $(0.0006)$ & $(0.0006)$ \\
& $0.5755^{* * *}$ & $0.5942^{* * *}$ \\
Capital Expenditure & $(0.0978)$ & $(0.1736)$ \\
& $0.0884^{* * *}$ & $0.1023^{* * *}$ \\
Acquisition Expenditure & $(0.0275)$ & $(0.0276)$ \\
& $0.03188^{* * *}$ & $0.0319^{* * *}$ \\
Dividend Paying Firms $(0 / 1)$ & $(0.0104)$ & $(0.0109)$ \\
& $-0.3534^{* * *}$ & $-0.4123^{* *}$ \\
RED Expenditure & $(0.1418)$ & $(0.2041)$ \\
& $0.2686^{* * *}$ & $0.3431^{* * *}$ \\
Profitability & $(0.0722)$ & $(0.0705)$ \\
& $0.6619^{* * *}$ & $0.7081^{* * *}$ \\
Cash & $(0.1380)$ & $(0.1604)$ \\
Observations & 1.1802 & 1.1845 \\
& $(0.4031)$ & $(0.4891)$ \\
& 42,893 & 42,893 \\
\hline
\end{tabular}


Table 1.6: Data breaches and cash holdings

This table reports the estimation results for the impact of data breaches on cash holdings. In Columns (1) and (2), we estimate Eq.(1.2), the effects of data breaches on cash holdings. In Columns (3) and (4), we estimate the effects on cash holdings of data breach severity. The variable Breach $(0 / 1)$ is set to one for breached firms in the year of the data breach, and zero otherwise; Severe Breach(0/1) proxies for the expected costs of an unusually severe breach and is a dummy set to one for a breach with CARs in the lowest tercile (most negative returns); Moderate Breach $(0 / 1)$ is set to one for a breach with CARs in the middle tercile; and Low Breach(0/1) is set to one for a breach with CARs in the highest tercile (least negative returns). Standard errors clustered by firm are shown in parentheses, with $1 \%, 5 \%$, and $10 \%$ levels of statistical significance denoted by $* * *, * *$, and $*$, respectively.

\begin{tabular}{|c|c|c|c|c|}
\hline \multirow[b]{2}{*}{ Variables } & \multicolumn{4}{|c|}{ Dependent Variable: Cash } \\
\hline & $(1)$ & $(2)$ & $(3)$ & (4) \\
\hline $\operatorname{Breach}(0 / 1)_{t}$ & $\begin{array}{c}0.0299 * * * \\
(0.0101)\end{array}$ & & & \\
\hline $\operatorname{Breach}(0 / 1)_{t-1}$ & & $\begin{array}{c}0.0282^{* * *} \\
(0.0104)\end{array}$ & & \\
\hline Severe Breach $(0 / 1)_{t}$ & & & $\begin{array}{l}0.0348^{* *} \\
(0.0151)\end{array}$ & \\
\hline Moderate Breach $(0 / 1)_{t}$ & & & $\begin{array}{l}0.0285^{*} \\
(0.0163)\end{array}$ & \\
\hline Low Breach $(0 / 1)_{t}$ & & & $\begin{array}{c}0.0224 \\
(0.0170)\end{array}$ & \\
\hline Severe Breach $(0 / 1)_{t-1}$ & & & & $\begin{array}{r}0.0379^{* *} \\
(0.0187)\end{array}$ \\
\hline Moderate Breach $(0 / 1)_{t-1}$ & & & & $\begin{array}{c}0.0230 \\
(0.0165)\end{array}$ \\
\hline Low Breach $(0 / 1)_{t-1}$ & & & & $\begin{array}{c}0.0198 \\
(0.0151)\end{array}$ \\
\hline Controls & Yes & Yes & Yes & Yes \\
\hline Year Fixed Effects & Yes & Yes & Yes & Yes \\
\hline Industry Fixed Effects & Yes & Yes & Yes & Yes \\
\hline Observations & 42,893 & 42,893 & 42,878 & 42,866 \\
\hline$A d j . R^{2}$ & 0.4981 & 0.4981 & 0.4982 & 0.4982 \\
\hline
\end{tabular}


Table 1.7: Data breaches and external financing

This table reports the estimation results for data breaches and external financing. The dependent variable in Columns (1) and (2) is external equity financing, which is estimated as the difference between the sale of common and preferred stocks and the purchase of common and preferred stocks, scaled by total assets at the beginning of the year. The dependent variable in Columns (3) and (4) is external debt financing, which is measured as the ratio of long-term debt issuance minus long-term debt reduction to total assets at the beginning of the year. The variable Breach(0/1) is set to one for breached firms in the year of the data breach, and zero otherwise; Severe Breach(0/1) proxies for the expected costs of an unusually bad breach and is a dummy set to one for a breach with CARs in the lowest tercile (most negative returns); Moderate Breach $(0 / 1)$ is set to one for a breach with CARs in the middle tercile; and Low Breach(0/1) is set to one for a breach with CARs in the highest tercile (least negative returns). Standard errors clustered by firm are shown in parentheses, with 1\%,5\%, and $10 \%$ levels of statistical significance denoted by $* * *, * *$, and $*$, respectively.

\begin{tabular}{lcccc}
\hline \hline & \multicolumn{3}{c}{ Dependent Variable: } & External financing \\
& External Equity Financing & External Debt Financing \\
Variables & $(1)$ & $(2)$ & $(3)$ & $(4)$ \\
\hline Breach $(0 / 1)$ & $-0.0140^{* * *}$ & \multicolumn{3}{c}{$-0.0171^{* * *}$} \\
Severe Breach $(0 / 1)$ & $(0.0051)$ & $(0.0048)$ & \\
& & $-0.0228^{* * *}$ & & $-0.0232^{* * *}$ \\
Moderate Breach $(0 / 1)$ & & $(0.0082)$ & & $(0.0076)$ \\
& & -0.0132 & & $-0.0179^{* *}$ \\
Low Breach(0/1) & & $(0.0081)$ & & $(0.0068)$ \\
& & -0.0056 & & $-0.0142^{*}$ \\
Controls & Yes & $(0.0077)$ & & $(0.0098)$ \\
Year Fixed Effects & Yes & Yes & Yes & Yes \\
Industry Fixed Effects & Yes & Yes & Yes & Yes \\
Observations & 42,893 & 42,893 & 42,893 & 42,893 \\
Adj. $R^{2}$ & 0.1270 & 0.3083 & 0.2550 & 0.2330 \\
\hline
\end{tabular}


Table 1.8: Data breaches and corporate investment

This table reports the estimation results for the impact of data breaches on corporate investment. In Columns (1) and (2), the dependent variable is Capital Expenditure, the ratio of capital expenditure to total book assets at the beginning of the year. In Columns (3) and (4), the dependent variable is Acquisition Expenditure, defined as the ratio of acquisitions to total book assets at the beginning of the year. The variable Breach (0/1) is set to one for breached firms in the year of the data breach, and zero otherwise; Severe Breach(0/1) proxies for the expected costs of an unusually bad breach and is a dummy set to one for a breach with CARs in the lowest tercile (most negative returns); Moderate Breach(0/1) is set to one for a breach with CARs in the middle tercile; and Low Breach(0/1) is set to one for a breach with CARs in the highest tercile (least negative returns). Standard errors clustered by firm are shown in parentheses, with $1 \%, 5 \%$, and $10 \%$ levels of statistical significance denoted by $* * *, * *$, and *, respectively.

\begin{tabular}{lcccc}
\hline \hline & \multicolumn{2}{c}{ Capital Expenditure } & \multicolumn{2}{c}{ Acquisitions } \\
Variables & $(1)$ & $(2)$ & $(3)$ & $(4)$ \\
\hline Breach $(0 / 1)$ & $-0.0072^{* *}$ & \multicolumn{3}{c}{$-0.0209^{* * *}$} \\
& $(0.0031)$ & $(0.0040)$ & \\
Severe Breach $(0 / 1)$ & & $-0.0092^{* *}$ & \multicolumn{2}{c}{$-0.0219^{* * *}$} \\
& & $(0.0043)$ & & $(0.0056)$ \\
Moderate Breach $(0 / 1)$ & & $-0.0074^{*}$ & & $-0.0211^{* * *}$ \\
& & $(0.0044)$ & & $(0.0062)$ \\
Low Breach $(0 / 1)$ & & -0.0068 & & $-0.0136^{*}$ \\
& & $(0.0053)$ & & $(0.0082)$ \\
Controls & Yes & Yes & Yes & Yes \\
Year Fixed Effects & Yes & Yes & Yes & Yes \\
Industry Fixed Effects & Yes & Yes & Yes & Yes \\
Observations & 42,893 & 42,893 & 42,893 & 42,893 \\
Adj. $R^{2}$ & 0.0237 & 0.0237 & 0.0221 & 0.0221 \\
\hline
\end{tabular}


Table 1.9: Variable definitions

This table presents the definitions of the key variables. The accounting data are from Compustat and the breach data are from the Privacy Rights Clearinghouse website (https://www.privacyrights.org).

\begin{tabular}{|c|c|}
\hline Variable & Definition \\
\hline Cash & $\begin{array}{l}\text { Cash and marketable securities scaled by total book as- } \\
\text { sets at the beginning of the year }\end{array}$ \\
\hline External Debt Financing & $\begin{array}{l}\text { Ratio of long-term debt issuance minus long-term debt } \\
\text { reduction to total assets at the beginning of the year }\end{array}$ \\
\hline External Equity Financing & $\begin{array}{l}\text { Ratio of the difference between the sale of common and } \\
\text { preferred stocks and the purchase of common and pre- } \\
\text { ferred stocks to total assets at the beginning of the year }\end{array}$ \\
\hline $\operatorname{Breach}(0 / 1)$ & $\begin{array}{l}1 \text { for breached firms in the year of the data breach, and } \\
0 \text { otherwise }\end{array}$ \\
\hline Severe Breach(0/1) & $\begin{array}{l}1 \text { for a breach with CARs in the lowest tercile (most } \\
\text { negative returns) }\end{array}$ \\
\hline Moderate Breach $(0 / 1)$ & 1 for a breach with CARs in the middle tercile \\
\hline Low Breach(0/1) & $\begin{array}{l}1 \text { for a breach with CARs in the highest tercile (least } \\
\text { negative returns) }\end{array}$ \\
\hline Disclosure $\operatorname{Law}(0 / 1)$ & $\begin{array}{l}1 \text { for periods after the enactment of the state-level data } \\
\text { breach notification laws, and } 0 \text { otherwise }\end{array}$ \\
\hline Firm Age & $\begin{array}{l}\text { Natural logarithm of the number of years a firm has been } \\
\text { listed in the merged CRSP/Compustat database }\end{array}$ \\
\hline Market-to-book & $\begin{array}{l}\text { Ratio of total book assets less the book value of common } \\
\text { equity plus the total market value of equity, all divided } \\
\text { by total book assets }\end{array}$ \\
\hline Firm Size & Natural logarithm of total book assets \\
\hline Book Leverage & $\begin{array}{l}\text { Ratio of total book debt (short-term debt plus long-term } \\
\text { debt) to total book assets }\end{array}$ \\
\hline Cash Flow & $\begin{array}{l}\text { Ratio of earnings after interest, dividends, and taxes but } \\
\text { before depreciation to book assets }\end{array}$ \\
\hline Capital Expenditure & $\begin{array}{l}\text { Ratio of capital expenditure to total book assets at the } \\
\text { beginning of the year }\end{array}$ \\
\hline Acquisition Expenditure & $\begin{array}{l}\text { Ratio of acquisitions to total book assets at the begin- } \\
\text { ning of the year }\end{array}$ \\
\hline Dividend Paying Firms (0/1) & $\begin{array}{l}1 \text { in the year a firm pays dividends, and } 0 \text { otherwise; set } \\
\text { to zero if missing }\end{array}$ \\
\hline RED Expenditure & $\begin{array}{l}\text { Ratio of R\&D expenses to total book assets at the be- } \\
\text { ginning of the year }\end{array}$ \\
\hline Net Working Capital & Ratio of net working capital to net assets \\
\hline Industry Cash Flow Volatility & $\begin{array}{l}\text { Standard deviation of industry average cash flows for } \\
\text { the previous } 10 \text { years; at least } 3 \text { years of observations } \\
\text { required }\end{array}$ \\
\hline
\end{tabular}


Chapter 2

Data breaches and firm credit risk 


\section{$2.1 \quad$ Introduction}

Data breaches are a growing concern with millions of consumer records compromised and thousands of breaches reported annually. The impact of data breaches extends beyond the direct costs associated with breach investigation and remediation. Prior research shows that data breaches have important implications on firm value (Kamiya et al. (2020); Rosati et al. (2017); Gatzlaff and McCullough (2010)); litigation risk (Romanosky et al. (2014)); firm reputation and profitability (Lending et al. (2018); Akey et al. (2018)); insider trading (Lin et al. (2019)); financial and investment policies (Boasiako and Keefe (2020); Garg (2020)). In summary, data breaches cause substantial injury to firms and have the potential to hit the bottom line profitability of firms. In this paper, we extend the literature about data breaches and corporate finance by examining whether credit risk perceptions change towards firms that experience data breaches relative to non-breached firms.

Specifically, we examine whether firms exposed to data breaches experience increases in overall credit risk, as measured by firm-level credit ratings and the credit default swap (CDS) spread. The bond market is comprised of sophisticated information users who have an asymmetric loss function (Beaver et al. (2006)) and thus are incentivized to quickly assimilate negative information. Hence, credit rating agencies and CDS investors should be particularly sensitive to potential changes in firm risk profiles resulting from data breaches. Indeed, there is anecdotal evidence that suggests that data breaches can have large, negative impact on firm-level credit risk, and bond market participants factor data breach risks in credit risk assessments. For example, following the massive breach of Equifax's consumer database in 2017, Moody's downgraded Equifax rating outlook from stable to negative, citing data breach related costs as reasons for the downgrade. Moody's further announced that cyber risks are important and influential in credit ratings. Also, the parent company of AMCA filed for bankruptcy amid the fallout from its 2019 data breach incident. The company in its Chapter 11 filing cited the data breach and the 
enormous breach related expenses that were beyond its ability to bear as the reasons for the bankruptcy filing.

Understanding the impact of data breaches on firm credit risk is important. This is because data breaches affect firms' future performance, financial stability, and risk profiles. However, empirically, it remains unclear whether and to what extent data breaches are incorporated into firm credit risk assessments. We address this question by examining whether data breaches affect bond market participants' credit risk assessments. If bond market participants incorporate the impact of data breaches into credit risk assessments, then we predict increases in credit risk when data breaches occur. That is, credit ratings are likely to deteriorate and CDS spread is likely to increase following a data breach event. The occurrence of a data breach incident represents an external shock to the firm. That is, data breaches are an event that corporations and bond market participants have very little control over, including the occurrence, magnitude, and timing; they merely react to it. Hence, data breaches represent a unique type of corporate event, one that is less prone to endogeneity concerns than other types of corporate incidents. Our study provides insight into how bond market participants respond to data breach events through credit ratings and CDS spreads.

Credit ratings and CDS market constitute a meaningful setting for our research question because credit rating agencies are adept at incorporating "soft" information when assessing credit risk (Kraft (2015)). The public announcement of a data breach equals an unexpected expansion of what credit rating agencies know about the breached firm. Also, CDS spreads provide direct market estimates of credit losses, as opposed to bond yield spreads that in addition contain an inflation expectation and liquidity component (see, for instance, Longstaff et al. (2005)). CDS spreads appear to reflect available information on future credit losses well, as they tend to anticipate debt downgrades (Norden and Weber (2004)), and may reflect insider information (see Acharya and Johnson (2007)), as price discovery takes place primarily in the CDS market (see Blanco et al. (2005)). 
Imperatively, CDS spreads accurately anticipate the outcomes of uncertainty-inducing events that are later reflected by changes in firms' financial ratios (Kiesel et al. (2021)).

We construct a comprehensive data set covering firms exposed to data breaches to study the effects of data breaches on firm credit risk. Data breach incidents amongst publicly traded firms are obtained from the Privacy Rights Clearinghouse (PRC) database, a consumer advocacy not-for-profit organization that collates all disclosed data breaches in the United States. CDS spread data are from the Refinitiv database and firms' Standard \& Poor's (S\&P) monthly credit ratings data as well as data on firm characteristics are from Compustat. Over the 2009-2018 period, we identify 116 firm events of data breaches with complete CDS and credit rating data.

We test our hypotheses by examining whether increases in firm credit risk, as proxied for by firm-level credit ratings and CDS spreads are associated with data breaches by using a propensity-scored-matched sample to ensure that differences in firm characteristics between breached and non-breached firms do not drive the outcomes. The results of this study show that data breach risk exposure leads to lower credit ratings (credit rating downgrades). The result has strong economic importance. For instance, we calculate the predicted percentage change in the probability of a firm falling within an A rating due to a one unit increase in data breach (i.e., going from 0 to 1 ), while retaining all other variables at their mean values. We find that if data breach increases by one unit, the predicted percentage of firms rated A decreases from $23.12 \%$ to $15.36 \%$, implying an absolute decrease of about 7.76 or equivalently a $33.56 \%$ decrease.

In addition, we examine whether data breaches are associated with the probability of corporate bonds being perceived as assets of speculative grade. We find that corporate bonds of firms exposed to data breaches face a higher probability or risk of becoming fallen angels. Furthermore, using an event study methodology, we examine CDS market reaction around data breach events and find that data breaches lead to a positive and significant cumulative CDS spread. For instance, we find an average 3-day cumulative CDS spread change of 7.2 basis points around data breach announcements. A multivariate 
analysis of the test on the effect of data breaches on CDS spread shows that data breaches have positive and significant effect on CDS spread, which is consistent with the notion that CDS investors view data breaches as credit risk increasing event.

As explained earlier, CDS spreads accurately anticipate the outcomes of uncertaintyinducing events that are later reflected by changes in firms' financial ratios (Kiesel et al. (2021)). So, we explore the likely channels through which the changes in firm credit risk arise by examining the effect of data breaches on firm profitability and financial distress. First, we examine the effect of data breaches on firm operating performance and financial health. Data breach poses risk to a firm's profitability and future operations through changes in financial performance and financial distress. The reasons include increased litigation risk (Romanosky et al. (2014)); reputation damages and actions to rebuild reputation such as increase IT security spend and corporate social responsibility (Akey et al. (2018)); increased out-of-pocket costs (Kamiya et al. (2020)). We find a decrease in firm profitability, proxied by ROA and Sales Growth (likely indicating loss of customers) and increase in financial distress (decrease in Interest Coverage and Altman's Z) following a data breach. Also, credit rating agencies and CDS investors may reassess the probability of future data breaches, which could affect the credit risk assessment of breached firms. We examine the likelihood of a future data breach, conditional on a past data breach incident. We find that conditional on a past data breach incident, the likelihood of a second or future breach increases.

Our paper makes several key contributions to the corporate finance literature. It makes a timely contribution to the stream of studies that examine the economic consequences of data breaches. For example, Garg (2020) finds that data breaches not only affect the targeted firm but it also affects suppliers, peer firms defined by similar industry, and proximity. Kamiya et al. (2020); Gatzlaff and McCullough (2010) find significant negative stock market reactions to data breaches. Lin et al. (2019); Rosati et al. (2017) find that some insiders trade based on internal knowledge of data breaches to earn substantial profits. Lending et al. (2018) highlight the importance of examining 
the consequences of adverse data breach events and the potential actions of the corporate victims during the period after the adverse breach event. Those potential consequences include decreased sales, increased allowances for product returns, reduced compensation, and possible turnover.

Our paper is closest in spirit to Iyer et al. (2020) who use an event study methodology to examine bond yield spread reaction to data breaches. However, we delineate from these prior studies in several ways. First, we measure the market perception of credit quality and corporate default risk through both credit ratings and corporate credit default swap (CDS) spreads. Credit rating agencies are adept at incorporating "soft" information when assessing credit risk; and CDS instruments are transacted in derivative markets. The spreads represent the payments that must be paid by the buyer of CDS to the seller for the contingent claim in the case of a credit event, in this case nonpayment (or forced restructuring) of corporate debt, and therefore price market-based default risk (Aizenman et al. (2013)). Hence, from an empirical standpoint, there are advantages of using CDS spreads rather than bond yield spreads as proxy for default risk. For instance, bond yield spreads embed inflation expectations and demand/supply for credit conditions as well as default risk. In this study, we are only interested in credit quality and default risk, hence, CDS spreads provide direct market estimates of credit losses, as opposed to bond yield spreads that in addition contain an inflation expectation component. In addition, CDS spreads contribute more to price discovery than bond yield (Kiesel et al. (2021)) and reflect available information on future credit losses well, as they tend to anticipate debt downgrades (Norden and Weber (2004)). Second, we examine the impact of data breaches on firm credit risk. In particular, we examine the relation between data breaches and the probability or risk of becoming a fallen angel. In a survey analysis of CFOs about the cost of capital, capital budgeting, and capital structure, Graham and Harvey (2001) find that one of the most important factors affecting debt policy is credit rating; hence, firms are very concerned about their credit ratings, which is an indication of concern about distress. Third, we explore the likely channels through which the changes in firm 
credit risk arise by examining the effect of data breaches on firm operating performance and financial health.

Furthermore, the paper adds new evidence to the corporate finance literature. To the best of our knowledge, our paper is the first to examine empirically whether credit rating agencies and credit default swap investors' credit risk perceptions change towards firms that experience data breaches relative to non-breached firms. We show that data breaches are important in credit risk assessments of firms.

The remainder of the paper is structured as follows. Section 2.2 develops the testable hypotheses. Section 2.3 describes the data, sample selection, and key variable construction. Section 2.4 describes the empirical specification. Section 2.5 reports the empirical findings, and Section 2.6 concludes the paper.

\subsection{Hypotheses}

CDS market investors and credit rating agencies are highly sophisticated bond market participants. While CDS investors typically consist of institutional investors, credit rating agencies have access to non-public information and are responsible for assessing firms' overall creditworthiness (Ham and Koharki (2016)). Furthermore, CDS spreads provide direct market estimates of credit losses (Demirguc-Kunt and Huizinga (2013)), reflect available information on future credit losses, as they tend to anticipate debt downgrades (Norden and Weber (2004)), and may reflect insider information (Acharya and Johnson (2007)). Because bond market participants have more incentive to assimilate negative information in risk assessment, CDS investors and credit rating agencies should be particularly sensitive to potential changes in firm risk profiles resulting from data breaches.

Besides direct costs, firms also face severe indirect costs of data breaches. Indirect costs of data breaches include an increase in the perceived uncertainty about the firms' prospects by investors, regulatory investigations and fines, litigation, media scrutiny and reputation damage, loss in the number of customers and suppliers, increased cash flow 
risks, and diversion of managers' time and resources (Boasiako and Keefe (2020)). Due to these costs, which could have a significant effect on risk profiles, profitability, financial stability, and firm's value, CDS investors will likely consider data breaches in credit risk analysis. Thus, CDS investors may consider data breach a substantial credit risk increasing event, which might lead to an increase CDS spread, everything else constant. Similarly, credit rating agencies are likely to include data breach risk exposure in their assessment of the credit worthiness of breached firms as a decline in profitability and firm value due to data breach has a direct effect on default risk. Thus we state our primary hypotheses:

Hypothesis 1. Data breach risk exposure leads to lower credit rating, everything else constant

Hypothesis 2. Data breach risk exposure increases CDS spread, everything else constant.

A key measure used for assessing credit risk is the interest coverage ratio. Firms with relatively low interest coverage are at greater risk to an adverse event such as data breach. This motivates our third hypothesis.

Hypothesis 3a. The effect of data breaches on firm-level credit rating is more pronounced for firms with low interest coverage, everything else constant.

Hypothesis $3 \mathbf{b}$. The effect of data breaches on CDS spread is more pronounced for firms with low interest coverage, everything else constant.

\subsection{Data and Sample Selection}

\subsubsection{Data collection}

We obtain records on data breaches of publicly traded firms, occurring between 2009 and 2018, from a chronological listing of disclosed data breaches from the Privacy Rights 
Clearinghouse (PRC) database (available at www.privacyrights.org). ${ }^{1} \mathrm{PRC}$ is a consumer advocacy not-for-profit organization that publishes data breaches disclosed in the United States. The PRC breach data includes name of breached firm, public announcement date of the breach, the number of records breached (if available), nature of breach and brief description of the breach, among other details. Because comprehensive CDS data is available from 2009, we restrict the final breach sample to firms that have corporate CDS spread and credit rating data over the 2009-2018 period. We identify 116 breached firms with complete data during this period.

Our CDS spread data is from the Refinitiv database, which provides daily corporate CDS spread data. The CDS spreads are provided across multiple maturities, available by currency type, and debt seniority. For the purpose of our study, we follow previous literature (Chang et al. (2015); Demirguc-Kunt and Huizinga (2013); Ismailescu and Kazemi (2010); Jorion and Zhang (2007)) and consider only 5-year CDS contracts as they constitute the majority of the entire CDS market. To keep the CDS spreads comparable, we require the sample to include only quotes for the senior unsecured debt tier that are denominated in US dollars, and we further restrict our CDS sample to CDS contracts with a Modified Restructuring documentation clause (Finnerty et al. (2013)). The Modified Restructuring clause was introduced in the International Swaps and Derivatives Association (ISDA) standard contract in 2001 to limit the scope of opportunistic behavior by sellers in the event of a restructuring agreement. The CDS spreads are reported in basis points, with a basis point equals to $\$ 1000$ to insure $\$ 10$ million of debt. For example, if the spread on a firm's CDS is 80 basis points, then an investor pays $\$ 80,000$ a year to insure against $\$ 10$ million worth of the company's debt. ${ }^{2}$ As default risk rises, so does the spread of the CDS.

\footnotetext{
${ }^{1}$ The PRC database has been used in prior studies such as Boasiako and Keefe (2020); Kamiya et al. (2020); Lending et al. (2018))

${ }^{2} 0.8 \%$ of notional amount needs to be paid each year, so $0.008 \times 10$ million $=\$ 80,000$ per year.
} 
For the analysis about credit ratings, we obtain firms' Standard \& Poor's (S\&P) monthly credit ratings data from Compustat. We also obtain data on firm characteristics from the Compustat database.

\subsubsection{Matched sample}

We construct a propensity-score-matched sample to ensure comparison is only between the breached firms (treatment group) and similar firms that have not been breached (control group). Unlike the case in which all non-breached firms are included in the Compustat sample as the control group, firms in the restricted propensity-score-matched sample are more comparable with one another. In addition, using a propensity-score-matched sample reduces potential biases in estimating causal effects stemming from correlations between the public announcement of data breaches and important firm characteristics. It also addresses the concern that our results could be driven by omitted variables that are correlated with the data breaches and the changes in firm credit risk. We implement the matching procedure as follows. For each breached firm, we identify control firms (5 nearest neighbors) that do not experience data breach using propensity score matching. The propensity score is calculated using logistic regression of Breach(0/1) (an indicator that takes the value 1 if a firm experiences a data breach in a given year, and 0 otherwise) on the basis of all firm characteristics (control variables) employed in this study. We then use the propensity scores to construct nearest-neighbor matched sample of firms, where for each breached firm, the 5 control firms with the closest propensity scores are chosen from the pool of non-breached firms. After dropping missing observations, this matching procedure provides a final sample of 2,412 annual CDS spread observations and 2,068 annual firm-level credit rating observations between 2009 and 2018. 


\subsubsection{Variables}

Following previous literature (e.g., Garg (2020); Boasiako and Keefe (2020); Kamiya et al. (2020)), we measure our variable of interest Breach(0/1) as a dummy variable that takes the value one if firm $i$ at time $t$ experiences a data breach; and zero otherwise.

As explained earlier, we use five-year CDS spread because they constitute the majority of the CDS market and provide the most accurate pricing estimate of the default risk for the underlying entity. Because Refinitiv provides daily CDS spreads, we follow previous studies (e.g., Ham and Koharki (2016)) and measure CDS Spread as the average of a firm's daily CDS spread in a given fiscal year. We use the daily spreads for the event study of the CDS market reaction to data breaches. Panel A of Table 2.1 reports descriptive statistics for the variables used in our analyses. The sample firms have a mean CDS spread of 172 basis points. This is consistent with Zhang et al. (2009) who report an average CDS spread of 172.40 basis points over the period 2001-2003. Panel B of Table 2.1 presents mean CDS spread segmented by the levels of data breach severity. Firms that reported a breach incident over the sample period have a mean CDS spread of 178 basis points. Firms that experienced severe breaches have a mean CDS spread of 183 basis points and those with low level of breach severity have a mean CDS spread of 176 basis points. ${ }^{3}$

We construct the main credit rating variable as in previous studies (e.g., Arena (2018); Ashbaugh-Skaife et al. (2006)) by converting credit ratings into numerical scores set to 7 for AAA ratings, 6 for AA ratings, 5 for A ratings, 4 for $\mathrm{BBB}$ ratings, 3 for $\mathrm{BB}$ ratings, 2 for $\mathrm{B}$ ratings, and 1 for $\mathrm{C}$ ratings. Because S\&P's firm-level credit ratings are provided monthly in Compustat, Credit Rating is the average of a firm's assigned monthly credit rating in a given fiscal year and rounded to the nearest integer (Ham and Koharki (2016)). As presented in Panel A of Table 2.1, the sample firms have a mean firm-level credit rating of 4.1, which falls within the BBB ratings on S\&P's credit rating scale. This is consistent

\footnotetext{
${ }^{3}$ Severe Breach(0/1) is set to one for breaches in the top CDS spread change distribution (high CDS spread increase), and Low Breach(0/1) is set to one for breaches in the bottom of the CDS spread change distribution (low CDS spread increase).
} 
with Arena (2018); Oikonomou et al. (2014); Beaver et al. (2006) who report average credit ratings between $\mathrm{BBB}$ and $\mathrm{BBB}-$, representing the lowest tier of investment grade bonds.

We include control variables for several firm characteristics that previous studies (Arena (2018); Ham and Koharki (2016)) find are related to credit rating and CDS spread including: Firm Size, Profitability, Book Leverage, Asset Tangibility, Market-tobook, Interest Coverage, Loss(0/1), and Capital Expenditure. To limit the influence of outliers, we winsorize all continuous variables at the top and bottom 1\%. A detailed description of the control variables is provided in Table 2.13. As presented in Panel A of Table 2.1, the sample firms are modestly leveraged with total debt (long-term debt plus short-term debt) encompassing $31 \%$ of total assets. Also, the sample firms exhibit reasonable financial strength with average interest coverage ratio of 11, operating losses of $11.5 \%$ of the time, and mean Altman Z-score of 9.6.

\subsection{Research Design}

As explained earlier, the bond market is comprised of sophisticated market participants, who due to an asymmetric loss function, better assimilate negative news than positive news. Hence, bond market participants are sensitive to negative events that could potentially change the risk profile of firms. Given this, we examine our hypotheses using the empirical specifications below.

First, to test Hypothesis 1, we estimate Eq. (2.1) using

$$
\text { Credit } \text { Rating }_{i, t}=\alpha+\beta \operatorname{Breach}(0 / 1)_{i, t}+\gamma X_{i, t}+\rho_{j}+\delta_{t}+\varepsilon_{i, t},
$$

an ordered logistic regression where $i, t$, and $j$, index firm, time, and industry, respectively. The dependent variable, Credit Rating, is S\&P's average annual rating, which is converted into a numerical score set to 7 for AAA ratings, 6 for AA ratings, 5 for A ratings, 4 for $\mathrm{BBB}$ ratings, 3 for $\mathrm{BB}$ ratings, 2 for $\mathrm{B}$ ratings, and 1 for $\mathrm{C}$ ratings. With this, the lower 
the credit rating score, the higher the credit risk. This definition of the dependent variable is similar to the one used in previous studies such as Arena (2018) and Ashbaugh-Skaife et al. (2006). The primary variable of interest in Eq. (2.1) is Breach(0/1), which is a dummy variable set to one if a firm $i$ discloses a data breach in time $t$; and zero otherwise. We expect the coefficient on $\operatorname{Breach}(0 / 1)$ to be positive $(\beta>0)$ if credit rating agencies increase their assigned credit risk assessments for firms that experience data breaches. $X_{i, t}$ is a vector of controls, which includes Firm Size, Profitability, Book Leverage, Asset Tangibility, Market-to-book, Interest Coverage, Loss(0/1), and Capital Expenditure. A detailed definition of these variables is provided in Table 2.13. $\rho_{j}$ and $\delta_{t}$ capture industry and time fixed effects, respectively.

In addition, to examine the effect of data breaches on the level of a firm's credit rating, we investigate the effect of data breaches on a possible downgrade or upgrade in credit rating. To test, we redefine the dependent variable, Credit Rating, to assume the value of -1 when a firm is downgraded to a lower credit rating from the previous year, 0 if it maintains the same credit rating from the previous year, and +1 if it is upgraded to a higher credit rating from the previous year. Here, with the exception of $\operatorname{Breach}(0 / 1)$, all the other independent variables are calculated as the difference between the current and previous year values.

We employ the standard event study methodology (Hull et al. (2004); Norden and Weber (2004)) to examine how CDS markets respond to data breach announcements. We use both the market model and the market-adjusted model in cumulative abnormal return (CAR) calculations. CDS spreads are, strictly speaking, not returns. They represent the incremental yield that compensates for bearing credit risk. As a result, we can use CDS spreads as a reasonable proxy for returns to calculate CARs. We compute change in CDS spread for the time interval $[t-1, t]$. For the market-adjusted model, we use the adjusted change in CDS spread to control the response measure for changes in global market conditions. The adjusted CDS spread is calculated as the difference between each 
firm's CDS spread change and the change in CDS index. ${ }^{4}$ For example, to calculate the adjusted CDS spread for Advanced Micro Devices (AMD), we create a CDS index, which is the average of the CDS spread of all sample firms with the same credit rating as AMD. We then calculate the adjusted CDS spread by applying the following formula:

$$
\text { Adjusted CDS } \text { Spread }_{i, t}=\Delta C D S \text { Spread }_{i, t}-\Delta C D S \text { Indext }_{t}
$$

where $\triangle C D S$ Spread $_{i, t}$ represents the change in CDS spread for firm $i$ at time $t . \triangle C D S$ Index is the change in index. This methodology is similar to the methodologies used in prior studies (Ismailescu and Kazemi (2010); Jorion and Zhang (2007);), which measure the adjusted CDS spread by subtracting the index from the firm's CDS spread to control for changes in CDS market conditions. We calculate CAR as $C A R_{i t}=\sum_{s=0}^{t} a C D S_{i s}$.

To test Hypothesis 2, we estimate

$$
C D S \text { Spread }_{i, t}=\alpha+\beta \operatorname{Breach}(0 / 1)_{i, t}+\gamma X_{i, t}+\rho_{j}+\delta_{t}+\varepsilon_{i, t},
$$

an OLS regression where $i, t$, and $j$, index firm, time, and industry, respectively. We follow Ham and Koharki (2016) and measure the dependent variable, CDS Spread, as the average of a firm's daily CDS spread in a given fiscal year. Here, CDS Spread is the five-year CDS spread and it is increasing in credit risk. All other variables maintain their definitions as explained earlier in Eq. (2.1).

To test Hypothesis 3a, we estimate

$$
\begin{aligned}
\text { Credit Rating }_{i, t} & =\alpha+\beta_{1} \text { Breach }(0 / 1)_{i, t}+\beta_{2} \operatorname{Breach}(0 / 1) \times \text { Low Interest Coverage }(0 / 1)_{i, t} \\
& +\gamma X_{i, t}+\rho_{j}+\delta_{t}+\varepsilon_{i, t},
\end{aligned}
$$

\footnotetext{
${ }^{4}$ The CDS index is the cross-sectional average of all CDS spreads in the sample within the same credit rating category.
} 
an ordered logistic regression where $i, t$, and $j$, index firm, time, and industry, respectively. The dependent variable, Credit Rating, is the numerical score explained in Eq. (2.1). As explained earlier, the lower the credit rating score, the higher the credit risk. We examine the impact of interest coverage on the relation between data breaches and firm-level credit rating. So, the primary variable of interest is the interaction term Breach $(0 / 1) \times$ Low Interest Coverage(0/1). We follow Gomariz and Ballesta (2014) and construct a low interest coverage indicator variable, which equals 1 if a firm belongs to the bottom of the interest coverage distribution, and 0 otherwise. The direct effect of low interest coverage is captured by a low interest coverage control variable. Because interest coverage is an important indicator of financial stress, the impact of data breaches on firm-level credit rating should be pronounced for breached firms with low interest coverage. Hence, we expect the coefficient on the interaction term Breach $(0 / 1) \times$ Low Interest Coverage $(0 / 1)$ to be positive $(\beta>0)$. All other variables maintain their previous definitions and robust standard errors are estimated by clustering at the firm level.

Finally, to test Hypothesis 3b, we estimate

$$
\begin{aligned}
& C D S \operatorname{Spread}_{i, t}=\alpha+\beta_{1} \operatorname{Breach}(0 / 1)_{i, t}+\beta_{2} \operatorname{Breach}(0 / 1) \times \text { Low Interest Coverage }(0 / 1)_{i, t} \\
& +\gamma X_{i, t}+\rho_{j}+\delta_{t}+\varepsilon_{i, t}
\end{aligned}
$$

an OLS regression where $i, t$, and $j$, index firm, time, and industry, respectively. The dependent variable, CDS Spread, remains the same as explained in Eq. (2.2). Here, we examine the impact of interest coverage on the relation between data breaches and corporate CDS spread. Firms with high interest coverage are likely to be rated investment grade. Firms rated investment grade face less financial constraints compared to speculative grade firms. They have healthier balance sheets and better access to credit markets in case of liquidity needs. Hence, the impact of data breaches on credit risk should be severe for firms with low interest coverage than firms with high interest coverage. So, 
the primary variable of interest is the interaction term Breach $(0 / 1) \times$ Low Interest Coverage(0/1). We expect the coefficient on the interaction term Breach $(0 / 1) \times$ Low Interest Coverage (0/1) to be positive $(\beta>0)$. As explained earlier, the direct effect of low interest coverage is captured by a low interest coverage indicator control variable. All other variables maintain their previous definitions and robust standard errors are estimated by clustering at the firm level.

\subsection{Empirical Results}

Our primary analyses examine whether credit rating agencies and CDS investors respond to data breaches. The multivariate analysis consists of tests on the effect of data breaches on the level of firm credit ratings, the change in credit ratings, credit rating grade, the CDS spread level, and the change in CDS spread. We also investigate the channels that may corroborate the empirical findings or reject alternative explanations.

\subsubsection{Data breaches and credit ratings}

Panel A of Table 2.2 presents the results of ordered logistic regressions with the credit rating numerical variable as dependent variable. We include year and industry fixed effects in the various regression specifications. The specifications also include control variables related to credit ratings as described in Section 2.3. Across Columns 1-3, the coefficient on Breach(0/1) is negative and statistically significant after controlling for corporate characteristics that affect firm credit ratings. These findings provide strong support for Hypothesis 1 and are consistent with the notion that credit rating agencies view data breach risk exposure as a credit risk increasing event, resulting in lower (i.e., worse) firm-level credit ratings. This result has also strong economic importance. To show the economic magnitude of data breaches on firm credit ratings, we use the results in Column (3) to calculate the predicted percentage change in the probability of a firm falling within an A rating due to a one unit increase in Breach(0/1) (i.e., going from 0 to 
1), while retaining all other variables at their mean values. If data breach increases by one unit (i.e., 0 to 1), the predicted percentage of firms rated A decreases from $23.12 \%$ to $15.36 \%$, implying an absolute decrease of about 7.76 or equivalently a $33.56 \%$ decrease.

In addition to examining the effect of data breaches on the level of a firm's credit rating, it is important to investigate the effect of data breaches on a possible downgrade or upgrade in credit rating. Boasiako and Keefe (2020) and Garg (2020) show that data breaches affect corporate cash policies. Therefore, the possible effect of data breaches on changes in credit ratings can have important implications on financial policies set by corporations. Panel B of Table 2.2 presents the results of ordered logistic regressions that analyze the effect of data breaches on possible credit rating changes when controlling for changes in firm characteristics from the previous year. The dependent indicator variable assumes the value of -1 when a firm is downgraded to a lower credit rating from the previous year, 0 if it maintains the same credit rating as the previous year, and +1 if it is upgraded to a higher credit rating from the previous year. With the exception of Breach (0/1), all the independent variables are calculated as the difference between the current and previous year values. Across Columns 1-3 of Panel B, the coefficient on Breach $(0 / 1)$ is negative and statistically significant, suggesting that data breaches lead to credit rating downgrade. Also, this result has strong economic importance. Using the results in Column (3) to calculate the predicted percentage change in the probability of a rating downgrade or upgrade due to a unit increase in data breach, we find that increasing data breach by one unit (i.e., 0 to 1), while keeping all other variables at their mean increases the predicted probability of a downgrade from $14.95 \%$ to $16.9 \%$ while decreases the probability of an upgrade from $15.29 \%$ to $13.5 \%$.

In Table 2.3, we examine whether data breaches are associated with the probability of corporate bonds being perceived as assets of speculative grade. ${ }^{5}$ The test consists of probit regressions with a dependent indicator variable equal to 1 when the bond is rated speculative grade and 0 when it is rated investment grade. The results on the relation

\footnotetext{
${ }^{5}$ Speculative grade bonds are bonds with less than triple B ratings
} 
between data breaches and probability of being perceived speculative grade present an analogous picture. Across columns 1-3, the coefficient on Breach(0/1) is positive and statistically significant, which suggests that corporate bonds of firms exposed to data breaches face a higher probability or risk of becoming fallen angels.

\subsubsection{Data breaches and credit default swap (CDS) spreads}

In this section, we examine the effect of data breaches on CDS spread. We begin by examining the reaction of CDS markets to data breaches using event study methodology. We then proceed to examine the relation between data breaches and CDS spread in a multivariate regression framework.

\section{CDS market reaction to data breaches}

We employ the standard event study methodology (Hull et al. (2004); Norden and Weber (2004)) to examine how CDS markets respond to data breach announcements. Table 2.4 presents the event study results of whether CDS markets respond to data breaches. We calculate CDS spread changes during the 3-day, 5-day, and 7-day event windows around the data breach announcements. We use both the market model and market-adjusted models to calculate the CARs. In both Panels A and B, the results show that data breaches lead, on average, to a statistically significant positive impact on CDS spread. For instance, using the market model in Panel A, we find an average 3-day CDS spread change of 7.2 during a data breach announcement. ${ }^{6}$ This result has strong economic importance. All else being equal, an increase in CDS spread by 7.2 during the 3-day event window corresponds to a $4.2 \%$ increase from mean CDS spread (172).

\section{Data breaches and CDS spread}

Table 2.5 presents the results from estimating Eq. (2.2) using OLS regressions. In Columns 1 and 2, the dependent variable is CDS spread in basis points. In Column 3,

\footnotetext{
${ }^{6}$ The results are qualitatively similar when we use alternative windows of 5 days and 7 days.
} 
we follow Oikonomou et al. (2014) and compute the natural logarithm of CDS spread to adjust for potential skewness in the CDS spread distribution and use it as the dependent variable. ${ }^{7}$ In Column 4, the dependent variable is the change in annual average CDS spread $\triangle C D S$ Spread. In Column 4, all the independent variables with the exception of Breach(0/1) are calculated as the difference between the current and previous year values. We include year and industry fixed effects in various specifications. Across Columns 1-4, the coefficient on $\operatorname{Breach}(0 / 1)$ is positive and statistically significant. This result is economically important; we use the result in Column (2) to gauge the economic importance. The coefficient associated with Breach(0/1) is 31.2097. All else being equal, an increase in CDS spread by 31.2097 corresponds to a $18.15 \%$ increase from mean CDS spread (172) for our sample firms. This result supports the credit rating findings reported earlier and is consistent with the notion that CDS investors view data breaches as credit risk increasing event, resulting in higher CDS spreads for breached firms.

\subsubsection{The impact of interest coverage}

In this section, we examine the impact of interest coverage on the relation between data breaches and firm credit risk. Interest coverage ratio incorporates the effects of both business risk and financial risk, hence, a key component of the bond market's pricing mechanism and rating decision (Foss (1995)).

In Table 2.6, we examine the effect of interest coverage on the relation between data breaches and firm-level credit ratings. As explained earlier in Section 2.4, we construct an interaction term $($ Breach $(0 / 1) \times$ Low Interest Coverage $(0 / 1))$, which is the variable of interest. The low interest coverage indicator variable equals 1 if a firm belongs to the bottom of the interest coverage distribution, and 0 otherwise. Across Columns 1-3 of Table 2.6, the coefficient on Breach $(0 / 1) \times$ Low Interest Coverage(0/1) is negative and statistically significant at the $5 \%$ level of significance, confirming that the effect of data

\footnotetext{
${ }^{7}$ In our sample, the natural logarithm of CDS spread has a kurtosis of 2.9 (which is close to the expected value of 3 ) and skewness of 0.06 .
} 
breach on firm-level credit ratings is particularly strong or more pronounced for firms with low interest coverage. For firms with low interest coverage, a data breach incident decreases the predicted probability of having an A rating from $20.95 \%$ to $9.39 \%$, implying an absolute decrease of about 11.56 or equivalently a $55 \%$ decrease.

In Table 2.7, we examine the impact of interest coverage on the relation between data breaches and corporate CDS spread. If a firm has a very high interest coverage then it is likely to be rated investment grade. Firms rated investment grade face less financial constraints compared to speculative grade firms. They have healthier balance sheets and better access to credit markets in case of liquidity needs. Hence, the impact of data breaches on credit risk should be severe for firms with low interest coverage than firms with

high interest coverage. Across Columns 1-4 of Table 2.7, the regression coefficient of the interaction term between the breach indicator and the low interest coverage indicator is positive and statistically significant, and larger than the coefficient of the breach indicator from the stand-alone effects of data breaches on CDS spread presented earlier, confirming that the effect of data breach on CDS spread is particularly strong or more pronounced for firms with low interest coverage.

\subsubsection{Mechanism}

Why do credit rating agencies and CDS markets alter their credit risk assessment after a data breach, by lowering credit ratings and charging higher CDS premiums. Prior research (Kiesel et al. (2021)) show that CDS spreads accurately anticipate the outcomes of risk-inducing events before they are reflected by changes in firms' financial ratios. In this section, we explore channels that may corroborate the empirical findings or reject alternative explanations.

\section{Data breach, profitability, and financial distress}

First, we study the effect of data breaches on firm operating performance and financial health. Data breach poses risk to a firm's future operations through changes in finan- 
cial performance and financial health. The reasons may include increased litigation risk (Romanosky et al. (2014)); reputation damages and actions to rebuild reputation such as increase IT security spend, infrastructure costs, and corporate social responsibility (Akey et al. (2018)); increased out-of-pocket cost (investigation and remediation cost, and fines and penalties from regulators) and increased costs of revamping overall risk management policies (Kamiya et al. (2020)).

Table 2.8 presents the results on the effect of data breaches on firm financial outcomes. The results on the relation between data breaches and operating performance are reported in Panel A of Table 2.8. The dependent variable in Columns 1 and 2 is Profitability (ROA) and Sales Growth in Columns 3 and 4. In Columns 1 and 3, the coefficient on breach is negative and significant, suggesting that in general, ROA and sales growth decline following a data breach. However, the decrease in ROA and sales growth exhibits substantial variation: severely breached firms experience significant decline in ROA and sales growth, while non-severe breached firms do not. ${ }^{8}$ Panel B presents the results for the relation between data breaches and financial distress. The dependent variable in Columns 1 and 2 is Interest Coverage and Altman's $Z$ in Columns 3 and 4 . A firm with higher interest coverage has the capability to service its debt, hence, faces lower bankruptcy risk. Firms with a higher Z-score have better financial health and lower default risk. Therefore, firms with lower interest coverage and Z-score should have a higher credit risk. The results show an increase in financial distress of firms following a data breach incident and the deterioration in financial health is even higher for severely breached firms.

\section{Conditional likelihood of additional data breaches}

Credit rating agencies and CDS investors may reassess the probability of future data breaches, which could affect the credit risk assessment of breached firms. In Table 2.9,

\footnotetext{
${ }^{8}$ Severe Breach(0/1) is set to one for breaches in the top CDS spread change distribution (high CDS spread increase), and Low Breach(0/1) is set to one for breaches in the bottom of the CDS spread change distribution (low CDS spread increase).
} 
we follow Binfare (2019) and use probit regressions to examine the likelihood of a future data breach, conditional on a data breach occurring in year $\mathrm{t}$ or $\mathrm{t}-1$. The dependent variable is an indicator variable that takes a value of one if a firm experiences a data breach in year $t+1$, zero otherwise. The independent variables are $\operatorname{Breach}(0 / 1)_{t}$, which equals one if a breach occurs in year $t$, zero otherwise, and Breach $(0 / 1)_{t-1}$, which equals one if a breach is reported in year $t-1$, zero otherwise. Across Columns 1-4, we find positive and significant coefficients, suggesting that, conditional on a data breach incident, the likelihood of a second breach increases.

\subsubsection{Robustness tests}

\section{Robustness: CDS market reaction to data breach announcement}

As a robustness check, we examine how CDS markets respond to data breach announcements over longer event windows. Table 2.10 presents the event study results over 15-day and 30-day event windows. On average, we find CDS spread change of 4.2 and 1.5 over the 15-day and 30-day event windows, respectively. This further suggests that data breaches lead, on average, to a statistically significant positive impact on CDS spread even over longer event windows.

\section{Robustness: Data breach and profitability}

As a robustness check, we examine the effect of data breach on firm profitability in the year after the data breach. Table 2.11 presents the results. ROA is the dependent variable in Columns (1) and (2) and Sales is the dependent variable in Columns (3) and (4). The results are consistent with the previous findings. In Columns 1 and 3, the coefficient on breach is negative and significant, suggesting that in general, ROA and sales growth in the year following the breach decline, and severely breached firms experience significant decline in ROA and sales growth compared to non-severe breached firms. 


\section{Robustness: Interest coverage and the effect of data breaches on credit risk}

To test the robustness of the baseline results on the effect of interest coverage, we examine the impact of interest coverage on the relation between data breaches and firm credit risk by using interest coverage as a continuous variable as opposed to the dummy in the initial analysis. Table 2.12 presents the results. The dependent variable in Column 1 is the credit rating numerical variable and the dependent variable in Column 2 is CDS spread.

Across Columns 1 and 2, the coefficient of the interaction term, Breach $(0 / 1) \times$ Interest Coverage is the variable of interest. In Column 1, the first row shows that the coefficient on Breach(0/1) is negative and similar to the baseline results. That is, a data breach event leads to decline in credit ratings, ceteris paribus. The second row of Column 1 reports the coefficient on the main variable of interest, the interaction between breach and interest coverage. The sign of the coefficient is positive and opposite the sign of the coefficient on the main effect of Breach(0/1). This infers that, an increase in interest coverage moderates the relation between breach and credit rating. Hence, the relation between breach and credit rating is significantly stronger among lower interest coverage firms. In Column 2 (where the dependent variable is CDS spread), the sign of the coefficient of the interaction term, Breach $(0 / 1) \times$ Interest Coverage is negative and opposite the sign of the coefficient on the main effect of Breach(0/1). Again, this infers that an increase in interest coverage moderates the relation between breach and CDS spread. Hence, the relation between breach and CDS spread is significantly stronger among lower interest coverage firms.

\subsection{Conclusion}

The bond market is comprised of sophisticated market participants with an asymmetric loss function, hence, should be particularly sensitive to events that could potentially change the risk profile of firms. As data breaches are increasing in frequency and severity, 
it is important to fully understand how data breaches affect a firm's credit risk. This paper examines whether bond market participants' (credit ratting agencies and CDS investors) credit risk perceptions change towards firms that experience data breaches relative to similar non-breached firms. Specifically, we examine whether breached firms experience decline in credit ratings and increase in CDS premiums. The results of this paper suggest that bond market participants' credit risk perceptions change towards breached firms. Firms experience a decline in credit ratings when they are exposed to data breaches and the decline in credit ratings is more pronounced for breached firms with low interest coverage. Also, corporate bonds of firms exposed to data breaches face a higher probability of being perceived as assets of speculative grade. Furthermore, data breaches lead to significant increase in CDS spread and are even higher for breached firms with low interest coverage. We explore the channels that corroborate these results and find that data breaches lead to a decline in operating performance, an increase in distress, and increased likelihood of future breaches. 


\section{Bibliography}

Acharya, V. V. and Johnson, T. C. (2007). Insider trading in credit derivatives. Journal of Financial Economics, 84(1):110-141.

Aizenman, J., Hutchison, M., and Jinjarak, Y. (2013). What is the risk of european sovereign debt defaults? fiscal space, cds spreads and market pricing of risk. Journal of International Money and Finance, 34:37-59.

Akey, P., Lewellen, S., and Liskovich, I. (2018). Hacking corporate reputations. Rotman School of Management Working Paper.

Arena, M. P. (2018). Corporate litigation and debt. Journal of Banking \& Finance, $87(1): 202-215$.

Ashbaugh-Skaife, H., Collins, D. W., and LaFond, R. (2006). The effects of corporate governance on firms' credit ratings. Journal of Accounting and Economics, 42(1-2):203243.

Beaver, W. H., Shakespeare, C., and Soliman, M. T. (2006). Differential properties in the ratings of certified versus non-certified bond-rating agencies. Journal of Accounting and Economics, 42(3):303-334.

Binfare, M. (2019). Corporate reputation, debt contracting, and bank loans: Evidence from data breaches. Working Paper.

Blanco, R., Brennan, S., and Marsh, I. W. (2005). An empirical analysis of the dynamic relationship between investment grade bonds and credit default swaps. The Journal of Finance, 60(5).

Boasiako, K. A. and Keefe, M. O. (2020). Data breaches and corporate liquidity management. European Financial Management, Forthcoming.

Chang, J.-H., Hung, M.-W., and Tsai, F.-T. (2015). Credit contagion and competitive effects of bond rating downgrades along the supply chain. Finance Research Letters, $15(1): 232-238$.

Demirguc-Kunt, A. and Huizinga, H. (2013). Are banks too big to fail or too big to save? international evidence from equity prices and cds spreads. Journal of Banking E Finance, 37(3):875-894. 
Finnerty, J. D., Miller, C. D., and Chen, R.-R. (2013). The impact of credit rating announcements on credit default swap spreads. Journal of Banking \& Finance, 37(6):2011-2030.

Foss, G. W. (1995). Quantifying risk in the corporate bond markets. Financial Analysts Journal, 51(2):29-34.

Garg, P. (2020). Cybersecurity breaches and cash holdings: Spillover effect. Financial Management, 49(2):503-519.

Gatzlaff, K. M. and McCullough, K. A. (2010). The effect of data breaches on shareholder wealth. Risk Management and Insurance Review, 13(1):61-83.

Graham, J. R. and Harvey, C. R. (2001). The theory and practice of corporate finance: Evidence from the field. Journal of Financial Economics, 60(2-3):187-243.

Gomariz, M.F.C. and Ballesta, J.P.S. (2014). Financial reporting quality, debt maturity and investment efficiency. Journal of Banking \& Finance, 40:494-506.

Ham, C. and Koharki, K. (2016). The association between corporate general counsel and firm credit risk. Journal of Accounting and Economics, 61(2-3):274-293.

Hull, J., Predescu, M., and White, A. (2004). The relationship between credit default swap spreads, bond yields, and credit rating announcements. Journal of Banking 86 Finance, 28(11):2789-2811.

Ismailescu, I. and Kazemi, H. (2010). The reaction of emerging market credit default swap spreads to sovereign credit rating changes. Journal of Banking \& Finance, 34(12):28612873.

Iyer, S. R., Simkins, B. J., and Wang, H. (2020). Cyberattacks and impact on bond valuation. Finance Research Letters, 33.

Jorion, P. and Zhang, G. (2007). Good and bad credit contagion: Evidence from credit default swaps. Journal of Financial Economics, 84(3):860-883.

Kamiya, S., Kang, J.-K., Kim, J., Milidonis, A., and Stulz, R. M. (2020). Risk management, firm reputation, and the impact of successful cyberattacks on target firms. Journal of Financial Economics, Forthcoming.

Kiesel, F., Kolaric, S., Norden, L., and Schiereck, D. (2021). To change or not to change? the cds market response of firms on credit watch. Journal of Banking $\&$ Finance, $125(1): 1-20$.

Kraft, P. (2015). Rating agency adjustments to gaap financial statements and their effect on ratings and credit spreads. The Accounting Review, 90(2):641-674.

Lending, C., Minnick, K., and Schorno, P. J. (2018). Corporate governance, social responsibility, and data breaches. Financial Review, 53(2):413-455. 
Lin, Z., Sapp, T., Ulmer, J. R., and Parsa, R. (2019). Insider trading ahead of cyber breach announcements. Journal of Financial Markets, 50:1-15.

Longstaff, F. A., Mithal, S., and Neis, E. (2005). Corporate yield spreads: Default risk or liquidity? new evidence from the credit default swap market. The Journal of Finance, 60(5):2213-2253.

Norden, L. and Weber, M. (2004). Informational efficiency of credit default swap and stock markets: The impact of credit rating announcements. Journal of Banking $\&$ Finance, 28(11):2813-2843.

Oikonomou, I., Brooks, C., and Pavelin, S. (2014). The effects of corporate social performance on the cost of corporate debt and credit ratings. Financial Review, 49(1):49-75.

Romanosky, S., Hoffman, D., and Acquisti, A. (2014). Empirical analysis of data breach litigation. Journal of Empirical Legal Studies, 11(1):74-104.

Rosati, P., Cummins, M., Deeney, P., Gogolin, F., Werff, L., and Lynn, T. (2017). The effect of data breach announcements beyond the stock price: Empirical evidence on market activity. International Review of Financial Analysis, 49(1):146-154.

Zhang, B. Y., Zhou, H., and Zhu, H. (2009). Explaining credit default swap spreads with the equity volatility and jump risks of individual firms. The Review of Financial Studies, 22(12):5099-5131. 
Table 2.1: Summary statistics

This table presents descriptive statistics for the variables used in our analyses. Panel A presents the descriptive statistics for the annual credit risk and firm characteristics of the full sample. Panel B presents the mean CDS spread for the breached firms and for the breach severity level. Panel C presents the mean CDS spread for the daily CDS observations for the full sample over the sample period. All variables are described in Table 2.13. To limit the influence of outliers, we winsorize all continuous variables at the 1 st and 99 th percentiles.

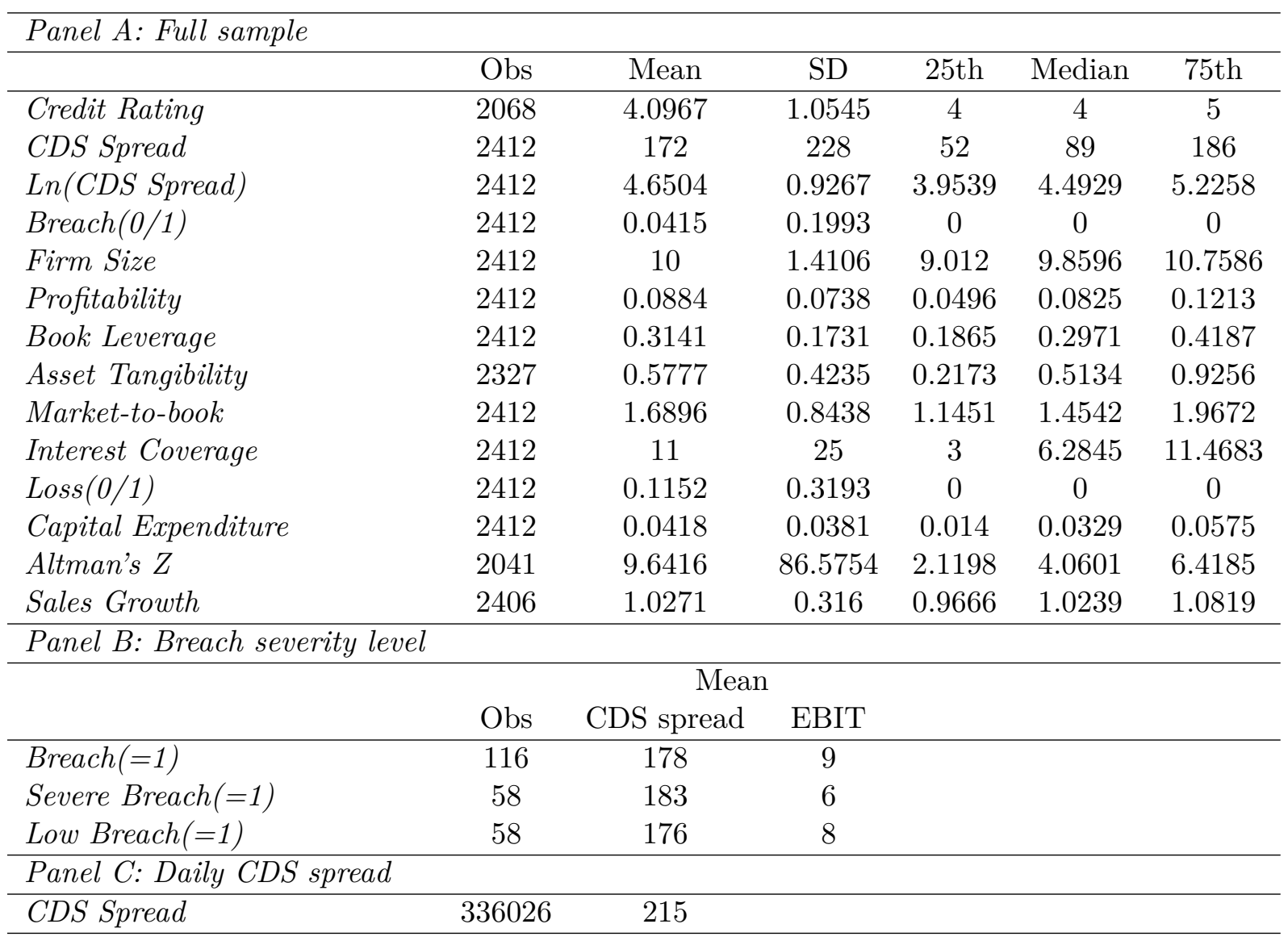


Table 2.2: Data breaches and credit ratings

This table presents multivariate tests about the relation between data breaches and credit ratings. Panel A presents ordered logistic regressions with credit rating as the dependent variable. Credit rating is a numerical score (i.e., 7 for AAA rating, 6 for AA ratings, 5 for A ratings, etc.). Panel B presents ordered logistic regressions with a dependent indicator variable equal to -1 when a firm is downgraded to a lower credit rating from the previous year, 0 if it maintains the same credit rating as the previous year, and +1 if it is upgraded to a higher credit rating from the previous year. In Panel B, all independent variables aside from the breach dummy are the difference between the current and previous year values. To limit the influence of outliers, we winsorize all continuous variables at the 1st and 99th percentiles. Standard errors are clustered by firm, p-values are reported in parentheses with less than $1 \%, 5 \%$, and $10 \%$ levels of statistical significance denoted by $* * *, * *$, and ${ }^{*}$, respectively.

\begin{tabular}{|c|c|c|c|}
\hline \multicolumn{4}{|l|}{ Panel A: Credit ratings } \\
\hline & $(1)$ & $(2)$ & $(3)$ \\
\hline $\operatorname{Breach}(0 / 1)$ & $\begin{array}{c}-0.7106^{* *} \\
(0.0144)\end{array}$ & $\begin{array}{c}-0.6642^{* *} \\
(0.0241)\end{array}$ & $\begin{array}{l}-0.5086^{*} \\
(0.0788)\end{array}$ \\
\hline Firm Size & $\begin{array}{c}1.2502^{* * * *} \\
(0.0000)\end{array}$ & $\begin{array}{c}1.2659^{* * *} \\
(0.0000)\end{array}$ & $\begin{array}{c}1.3219^{* * *} \\
(0.0000)\end{array}$ \\
\hline Profitability & $\begin{array}{l}5.3741^{*} \\
(0.0557)\end{array}$ & $\begin{array}{l}5.0256^{*} \\
(0.0832)\end{array}$ & $\begin{array}{l}5.3901^{*} \\
(0.0674)\end{array}$ \\
\hline Book Leverage & $\begin{array}{c}-4.7601^{* * *} \\
(0.0000)\end{array}$ & $\begin{array}{c}-4.7167^{* * *} \\
(0.0000)\end{array}$ & $\begin{array}{c}-4.3957^{* * *} \\
(0.0000)\end{array}$ \\
\hline Asset Tangibility & $\begin{array}{c}0.0642 \\
(0.8599)\end{array}$ & $\begin{array}{c}0.0396 \\
(0.9140)\end{array}$ & $\begin{array}{l}-0.0767 \\
(0.8389)\end{array}$ \\
\hline Market-to-book & $\begin{array}{c}0.9356^{* * *} \\
(0.0005)\end{array}$ & $\begin{array}{c}0.9995^{* * *} \\
(0.0006)\end{array}$ & $\begin{array}{c}0.9640^{* * *} \\
(0.0006)\end{array}$ \\
\hline Interest Coverage & $\begin{array}{c}0.0162^{* * *} \\
(0.0082)\end{array}$ & $\begin{array}{c}0.0162^{* * *} \\
(0.0075)\end{array}$ & $\begin{array}{l}0.0167^{* *} \\
(0.0105)\end{array}$ \\
\hline $\operatorname{Loss}(0 / 1)$ & $\begin{array}{c}-1.0295^{* * *} \\
(0.0000)\end{array}$ & $\begin{array}{c}-1.0748^{* * *} \\
(0.0000)\end{array}$ & $\begin{array}{c}-1.2495^{* * *} \\
(0.0000)\end{array}$ \\
\hline Capital Expenditure & $\begin{array}{c}2.4108 \\
(0.4905)\end{array}$ & $\begin{array}{c}2.7491 \\
(0.4399)\end{array}$ & $\begin{array}{c}2.6380 \\
(0.4822)\end{array}$ \\
\hline Year Fixed Effects & No & Yes & Yes \\
\hline Industry Fixed Effects & No & No & Yes \\
\hline Observations & 1,993 & 1,993 & 1,993 \\
\hline Pseudo $R^{2}$ & 0.2743 & 0.2771 & 0.2931 \\
\hline Panel B: Data breach and credit rating upgrade/downgrade & $(1)$ & $(2)$ & $(3)$ \\
\hline $\operatorname{Breach}(0 / 1)$ & $\begin{array}{c}-0.1903^{* *} \\
(0.0215)\end{array}$ & $\begin{array}{c}-0.1043^{* *} \\
(0.0426)\end{array}$ & $\begin{array}{l}-0.1250^{*} \\
(0.0646)\end{array}$ \\
\hline$\Delta$ Firm Size & $\begin{array}{c}-1.1238^{* *} \\
(0.0202)\end{array}$ & $\begin{array}{c}-1.2986^{* *} \\
(0.0105)\end{array}$ & $\begin{array}{c}-1.2981^{* *} \\
(0.0110)\end{array}$ \\
\hline$\Delta$ Profitability & $\begin{array}{l}-0.7248 \\
(0.6597)\end{array}$ & $\begin{array}{l}-0.3309 \\
(0.8443)\end{array}$ & $\begin{array}{l}-0.2602 \\
(0.8751)\end{array}$ \\
\hline$\Delta$ Book Leverage & $\begin{array}{c}1.3114 \\
(0.2094)\end{array}$ & $\begin{array}{l}2.1107^{*} \\
(0.0545)\end{array}$ & $\begin{array}{l}2.0019^{*} \\
(0.0681)\end{array}$ \\
\hline$\Delta$ Asset Tangibility & $\begin{array}{c}-1.9625^{* *} \\
(0.0413)\end{array}$ & $\begin{array}{c}-2.2967^{* *} \\
(0.0246)\end{array}$ & $\begin{array}{c}-2.1570^{* *} \\
(0.0362)\end{array}$ \\
\hline$\Delta$ Market-to-book & $\begin{array}{l}-0.0517 \\
(0.7675)\end{array}$ & $\begin{array}{c}-0.1333 \\
(0.4737)\end{array}$ & $\begin{array}{l}-0.1736 \\
(0.3575)\end{array}$ \\
\hline$\Delta$ Interest Coverage & $\begin{array}{l}-0.0011 \\
(0.2633)\end{array}$ & $\begin{array}{l}-0.0011 \\
(0.3398)\end{array}$ & $\begin{array}{l}-0.0008 \\
(0.4960)\end{array}$ \\
\hline$\Delta \operatorname{Loss}(0 / 1)$ & $\begin{array}{l}-0.1870 \\
(0.1902)\end{array}$ & $\begin{array}{l}-0.0922 \\
(0.5082)\end{array}$ & $\begin{array}{l}-0.0938 \\
(0.5015)\end{array}$ \\
\hline$\Delta$ Capital Expenditure & $\begin{array}{c}-9.8403^{* * *} \\
(0.0002)\end{array}$ & $\begin{array}{c}-6.1565^{* *} \\
(0.0303)\end{array}$ & $\begin{array}{c}-6.1709^{* *} \\
(0.0312)\end{array}$ \\
\hline $\begin{array}{l}\text { Year Fixed Effects } \\
\text { Industry Fixed Effects }\end{array}$ & $\begin{array}{l}\text { No } \\
\text { No }\end{array}$ & $\begin{array}{l}\text { Yes } \\
\text { No }\end{array}$ & $\begin{array}{l}\text { Yes } \\
\text { Yes }\end{array}$ \\
\hline
\end{tabular}


Table 2.3: Data breaches and probability of speculative grade rating

This table presents probit regressions with a dependent indicator variable equal to 1 when a firm is rated speculative grade, and 0 when it is rated investment grade. All other variables are described in Table 2.13. To limit the influence of outliers, we winsorize all continuous variables at the 1st and 99th percentiles. Standard errors are clustered by firm, p-values are reported in parentheses with less than 1\%, 5\%, and 10\% levels of statistical significance denoted by $* * *, * *$, and $*$, respectively.

\begin{tabular}{lccc}
\hline & \multicolumn{2}{c}{ Dependent } & Variable: Speculative Grade(0/1) \\
& $(1)$ & $(2)$ & $(3)$ \\
\hline Breach(0/1) & $0.4420^{* *}$ & $0.4344^{* *}$ & $0.3989^{*}$ \\
Firm Size & $(0.0377)$ & $(0.0420)$ & $(0.0681)$ \\
& $-0.6581^{* * *}$ & $-0.6744^{* * *}$ & $-0.6775^{* * *}$ \\
Profitability & $(0.0000)$ & $(0.0000)$ & $(0.0000)$ \\
& -0.9577 & -0.8061 & -0.7738 \\
Book Leverage & $(0.5222)$ & $(0.5969)$ & $(0.6133)$ \\
& $3.8869^{* * *}$ & $3.8868^{* * *}$ & $3.9205^{* * *}$ \\
Asset Tangibility & $(0.0000)$ & $(0.0000)$ & $(0.0000)$ \\
& 0.2916 & 0.3257 & 0.3591 \\
Market-to-book & $(0.3039)$ & $(0.2510)$ & $(0.2172)$ \\
& $-0.7994^{* * *}$ & $-0.8508^{* * *}$ & $-0.8499^{* * *}$ \\
Interest Coverage & $(0.0000)$ & $(0.0000)$ & $(0.0000)$ \\
& $0.0049^{* *}$ & $0.0052^{*}$ & $0.0047^{*}$ \\
Loss(0/1) & $(0.0448)$ & $(0.0531)$ & $(0.0851)$ \\
& $0.6161^{* * *}$ & $0.6425^{* * *}$ & $0.6508^{* * *}$ \\
Capital Expenditure & $(0.0003)$ & $(0.0002)$ & $(0.0001)$ \\
& -4.5414 & -5.0388 & $-5.1677^{*}$ \\
Year Fixed Effects & $(0.1343)$ & $(0.1003)$ & $(0.0842)$ \\
Industry Fixed Effects & No & Yes & Yes \\
Observations & 1,993 & No & Yes \\
Pseudo R $R^{2}$ & 0.3698 & 0.3752 & 1,993 \\
& & & 0.3804 \\
\hline
\end{tabular}


Table 2.4: CDS market reaction to data breaches

This table presents the event-study results of the impact of data breaches on CDS spread. We report the CDS spread changes of the 3-day, 5-day, and 7-day cumulative abnormal returns (CARs) during an event window of $(-1,+1),(-2,+2)$, and $(-3,+3)$ surrounding the data breach events. The standard deviations of the CDS spread changes are also presented in this table. We calculate CARs using both the market model and the market adjusted model. The adjusted CDS spread is the difference between the CDS spread and the benchmark index constructed as the cross-sectional average of all CDS spreads in the sample within the same credit rating category. ***, $* *$, and ${ }^{*}$ denote significance at the $1 \%, 5 \%$, and $10 \%$ levels, respectively.

\begin{tabular}{lccc}
\hline & CAR $(-1,1)$ & CAR $(-2,2)$ & CAR $(-3,3)$ \\
\hline Panel A: Market Model & & & \\
\hline CAR (bps) & $7.2^{* * *}$ & $8.4^{* * *}$ & $10.1^{* * *}$ \\
p-value & 0.000 & 0.000 & 0.000 \\
Standard Deviation & 16.5 & 22.9 & 28.3 \\
\hline Panel B: Market Adjusted Model & & & \\
\hline CAR (bps) & $6.7^{* * *}$ & $7.9^{* * *}$ & $9.2^{* * *}$ \\
p-value & 0.000 & 0.000 & 0.000 \\
Standard Deviation & 15.5 & 20.3 & 23.8 \\
\hline
\end{tabular}


Table 2.5: Data breaches and CDS spread

This table presents results about the relation between data breaches and CDS spread. In Columns 1 and 2 the dependent variable is CDS Spread in basis points. In Column 3 , the dependent variable is the natural logarithm of CDS Spread. In Column 4, the dependent variable is change in CDS spread, which is the change in annual average of CDS spread from year t-1 to year t, and all independent variables in Column 4 aside from the breach dummy are the difference between the current and previous year values. All other variables are described in Table 2.13. To limit the influence of outliers, we winsorize all continuous variables at the 1st and 99th percentiles. Standard errors are clustered by firm, p-values are reported in parentheses with less than $1 \%, 5 \%$, and $10 \%$ levels of statistical significance denoted by $* * *, * *$, and $*$, respectively.

\begin{tabular}{|c|c|c|c|c|}
\hline & \multirow{2}{*}{\multicolumn{2}{|c|}{$\begin{array}{l}\text { (1) } \\
\text { CDSSpread (bps) }\end{array}$}} & (3) & (4) \\
\hline & & & Ln(CDSSpread) & $\triangle C D S$ Spread \\
\hline \multirow{2}{*}{$\operatorname{Breach}(0 / 1)$} & $39.5512^{* * *}$ & $31.2097^{* *}$ & $0.1751^{* *}$ & $5.7336^{*}$ \\
\hline & $(0.0043)$ & $(0.0209)$ & $(0.0428)$ & $(0.0772)$ \\
\hline \multirow[t]{2}{*}{ Firm Size } & $-44.1330^{* * *}$ & $-44.2020^{* * *}$ & $-0.2788^{* * *}$ & 53.8183 \\
\hline & $(0.0000)$ & $(0.0000)$ & $(0.0000)$ & $(0.1203)$ \\
\hline \multirow[t]{2}{*}{ Profitability } & $-539.0416^{* *}$ & $-556.6948 * *$ & $-1.8055^{* *}$ & $-193.6334^{*}$ \\
\hline & $(0.0185)$ & $(0.0155)$ & $(0.0135)$ & $(0.0552)$ \\
\hline \multirow[t]{2}{*}{ Book Leverage } & $377.9864^{* * *}$ & $366.1522^{* * *}$ & $1.6203^{* * *}$ & $273.0070^{* *}$ \\
\hline & $(0.0000)$ & $(0.0000)$ & $(0.0000)$ & $(0.0198)$ \\
\hline \multirow[t]{2}{*}{ Asset Tangibility } & 33.7500 & 40.5052 & -0.0601 & $253.5598^{* * *}$ \\
\hline & $(0.3794)$ & $(0.3080)$ & $(0.6251)$ & $(0.0047)$ \\
\hline \multirow[t]{2}{*}{ Market-to-book } & $-34.2849^{* *}$ & $-34.0851^{* *}$ & $-0.2996 * * *$ & $-43.3957^{* * *}$ \\
\hline & $(0.0214)$ & $(0.0194)$ & $(0.0001)$ & $(0.0033)$ \\
\hline \multirow[t]{2}{*}{ Interest Coverage } & $0.4280^{*}$ & 0.3800 & -0.0003 & 0.1038 \\
\hline & $(0.0534)$ & $(0.1058)$ & $(0.7073)$ & $(0.3521)$ \\
\hline \multirow[t]{2}{*}{$\operatorname{Loss}(0 / 1)$} & $186.5323^{* * *}$ & $187.5633^{* * *}$ & $0.5808^{* * *}$ & $49.5101^{* * *}$ \\
\hline & $(0.0000)$ & $(0.0000)$ & $(0.0000)$ & $(0.0079)$ \\
\hline \multirow[t]{2}{*}{ Capital Expenditure } & $-821.3799^{* *}$ & $-836.6824^{* *}$ & -0.7178 & $-323.9204^{*}$ \\
\hline & $(0.0304)$ & $(0.0265)$ & $(0.5335)$ & $(0.0763)$ \\
\hline Year Fixed Effects & Yes & Yes & Yes & Yes \\
\hline Industry Fixed Effects & No & Yes & Yes & No \\
\hline Observations & 2,318 & 2,318 & 2,318 & 1,818 \\
\hline$R^{2}$ & 0.3815 & 0.3888 & 0.5217 & 0.1413 \\
\hline
\end{tabular}


Table 2.6: Interest coverage and the effect of data breaches on credit ratings

This table presents ordered logistic regressions about the role of interest coverage in the relation between data breaches and credit ratings. The dependent variable across Columns 1-3 is the credit rating numerical variable. Credit rating is a numerical score set to 7 for AAA ratings, 6 for AA ratings, 5 for A ratings, 4 for $\mathrm{BBB}$ ratings, 3 for $\mathrm{BB}$ ratings, 2 for $\mathrm{B}$ ratings, and 1 for $\mathrm{C}$ ratings. The variable of interest across Columns 1-3 is Breach $(0 / 1) \times$ Low Interest Coverage(0/1). All variables are described in Table 2.13. To limit the influence of outliers, we winsorize all continuous variables at the 1 st and 99th percentiles. Standard errors are clustered by firm, p-values are reported in parentheses with less than 1\%,5\%, and $10 \%$ levels of statistical significance denoted by $* * *, * *$, and $*$, respectively.

\begin{tabular}{lccc}
\hline & Dependent & Variable: & Credit Rating \\
& $(1)$ & $(2)$ & $(3)$ \\
\hline Breach(0/1) & -0.3836 & -0.3234 & -0.1609 \\
& $(0.2421)$ & $(0.3307)$ & $(0.6310)$ \\
Breach(0/1)×Low Interest Coverage $0 / 1)$ & $-0.8833^{* *}$ & $-0.9684^{* *}$ & $-0.9555^{* *}$ \\
& $(0.0226)$ & $(0.0239)$ & $(0.0229)$ \\
Firm Size & $1.2911^{* * *}$ & $1.3083^{* * *}$ & $1.3520^{* * *}$ \\
& $(0.0000)$ & $(0.0000)$ & $(0.0000)$ \\
Profitability & 3.3101 & 2.7909 & 3.6454 \\
& $(0.2139)$ & $(0.2949)$ & $(0.1811)$ \\
Book Leverage & $-3.9415^{* * *}$ & $-3.8673^{* * *}$ & $-3.5865^{* * *}$ \\
& $(0.0000)$ & $(0.0000)$ & $(0.0001)$ \\
Asset Tangibility & 0.1794 & 0.1662 & 0.0572 \\
& $(0.6327)$ & $(0.6615)$ & $(0.8832)$ \\
Market-to-book & $0.8831^{* * *}$ & $0.9652^{* * *}$ & $0.9349^{* * *}$ \\
& $(0.0004)$ & $(0.0003)$ & $(0.0003)$ \\
Low Interest Coverage(0/1) & $-1.1667^{* * *}$ & $-1.1641^{* * *}$ & $-1.1927^{* * *}$ \\
& $(0.0000)$ & $(0.0000)$ & $(0.0000)$ \\
Loss(0/1) & $-0.9168^{* * *}$ & $-0.9565^{* * *}$ & $-1.0769^{* * *}$ \\
Capital Expenditure & $(0.0001)$ & $(0.0001)$ & $(0.0000)$ \\
& 3.2319 & 3.3657 & 3.2665 \\
Year Fixed Effects & $(0.3705)$ & $(0.3610)$ & $(0.3988)$ \\
Industry Fixed Effects & No & Yes & Yes \\
Observations & No & No & Yes \\
Pseudo R ${ }^{2}$ & 1,993 & 1,993 & 1,993 \\
\hline & 0.2746 & 0.2775 & 0.2935 \\
\hline
\end{tabular}


Table 2.7: Interest coverage and the effect of data breaches on CDS spread

This table presents multivariate tests about the role of interest coverage in the relation between data breaches and CDS spread. In Columns 1 and 2 the dependent variable is CDS Spread in basis points. In Column 3, the dependent variable is the natural logarithm of CDS Spread. In Column 4, the dependent variable is change in CDS spread, which is the change in annual average of CDS spread from year t- 1 to year $t$, and all independent variables in Column 4 aside from the breach dummy and interest coverage dummy are the difference between the current and previous year values. The variable of interest across Columns $1-4$ is Breach $(0 / 1) \times$ Low Interest Coverage(0/1). All variables are described in Table 2.13. To limit the influence of outliers, we winsorize all continuous variables at the 1st and 99th percentiles. Standard errors are clustered by firm, p-values are reported in parentheses with less than 1\%, 5\%, and $10 \%$ levels of statistical significance denoted by ${ }^{* * *}, * *$, and $*$, respectively.

\begin{tabular}{lcccc}
\hline & $(1)$ & $(2)$ & $(3)$ & $(4)$ \\
& \multicolumn{1}{c}{ CDSSpread $(\mathrm{bps})$} & Ln $($ CDSSpread $)$ & $\Delta$ CDS Spread \\
\hline Breach $(0 / 1)$ & 22.3860 & 14.5005 & 0.0228 & $11.4149^{*}$ \\
& $(0.1213)$ & $(0.3140)$ & $(0.8247)$ & $(0.0723)$ \\
Breach $(0 / 1) \times$ Low Interest Coverage $(0 / 1)$ & $60.9833^{*}$ & 59.3422 & $0.5474^{* * *}$ & $21.4920^{* *}$ \\
& $(0.0968)$ & $(0.1114)$ & $(0.0045)$ & $(0.0313)$ \\
Firm Size & $-43.9830^{* * *}$ & $-44.0566^{* * *}$ & $-0.2777^{* * *}$ & 54.4961 \\
& $(0.0000)$ & $(0.0000)$ & $(0.0000)$ & $(0.1223)$ \\
Profitability & $-538.4124^{* *}$ & $-557.5671^{* *}$ & $-1.7597^{* *}$ & $-193.3784^{* *}$ \\
& $(0.0198)$ & $(0.0166)$ & $(0.0159)$ & $(0.0499)$ \\
Book Leverage & $373.2294^{* * *}$ & $361.2911^{* * *}$ & $1.5800^{* * *}$ & $250.5431^{* *}$ \\
& $(0.0000)$ & $(0.0000)$ & $(0.0000)$ & $(0.0352)$ \\
Asset Tangibility & 34.8330 & 41.5051 & -0.0513 & $261.4299^{* * *}$ \\
& $(0.3646)$ & $(0.2963)$ & $(0.6760)$ & $(0.0042)$ \\
Market-to-book & $-33.5378^{* *}$ & $-33.1103^{* *}$ & $-0.2978^{* * *}$ & $-48.9791^{* * *}$ \\
& $(0.0246)$ & $(0.0237)$ & $(0.0001)$ & $(0.0011)$ \\
Low Interest Coverage(0/1) & 0.4144 & 0.3672 & -0.0004 & 0.1432 \\
& $(0.0595)$ & $(0.1154)$ & $(0.6014)$ & $(0.2171)$ \\
Loss(0/1) & $186.6621^{* * *}$ & $187.6995^{* * *}$ & $0.5836^{* * *}$ & $48.6258^{* * *}$ \\
& $(0.0000)$ & $(0.0000)$ & $(0.0000)$ & $(0.0082)$ \\
Capital Expenditure & $-825.0334^{* *}$ & $-839.8297^{* *}$ & -0.7450 & $-312.4780^{*}$ \\
& $(0.0299)$ & $(0.0262)$ & $(0.5172)$ & $(0.0876)$ \\
Year Fixed Effects & Yes & Yes & Yes & Yes \\
Industry Fixed Effects & No & Yes & Yes & Yes \\
Observations & 2,318 & 2,318 & 2,318 & 1,818 \\
$R^{2}$ & 0.3816 & 0.3887 & 0.5235 & 0.1510 \\
\hline
\end{tabular}


Table 2.8: Data breach, profitability, and distress

This table presents results about the relation between data breaches and financial outcomes. Panel A presents results for data breaches and profitability with ROA as dependent variable in Columns (1) and (2) and Sales Growth as dependent variable in Columns (3) and (4). The variable Breach(0/1) is set to one for breached firms in the year of the data breach, and zero otherwise; Severe Breach(0/1) is set to one for breaches in the top CDS spread change distribution (high CDS spread increase), and Low Breach(0/1) is set to one for breaches in the bottom of the CDS spread change distribution (low CDS spread increase). Panel B presents results for data breaches and financial distress with Interest Coverage as dependent variable in Columns (1) and (2) Z-Score as dependent variable in Columns (3) and (4). All variables are described in Table 2.13. To limit the influence of outliers, we winsorize all continuous variables at the 1st and 99th percentiles. Standard errors are clustered by firm, p-values are reported in parentheses with less than 1\%,5\%, and $10 \%$ levels of statistical significance denoted by $* * *, * *$, and $*$, respectively.

\begin{tabular}{|c|c|c|c|c|}
\hline \multicolumn{5}{|l|}{ Panel A: Profitability } \\
\hline & \multicolumn{2}{|c|}{$\mathrm{ROA}$} & \multicolumn{2}{|c|}{ Sales } \\
\hline & (1) & $(2)$ & $(3)$ & (4) \\
\hline $\operatorname{Breach}(0 / 1)$ & $\begin{array}{c}-0.0061^{*} \\
(0.0703)\end{array}$ & & $\begin{array}{c}-0.0033^{*} \\
(0.0726)\end{array}$ & \\
\hline Severe Breach(0/1) & & $\begin{array}{c}-0.0127^{* *} \\
(0.0301)\end{array}$ & & $\begin{array}{c}-0.0157^{*} \\
(0.0618)\end{array}$ \\
\hline Low Breach(0/1) & & $\begin{array}{l}-0.0004 \\
(0.1443)\end{array}$ & & $\begin{array}{c}0.0083 \\
(0.2161)\end{array}$ \\
\hline Controls & Yes & Yes & Yes & Yes \\
\hline Year Fixed Effects & Yes & Yes & Yes & Yes \\
\hline Industry Fixed Effects & Yes & Yes & Yes & Yes \\
\hline Observations & 2,318 & 2,318 & 2,312 & 2,312 \\
\hline$R^{2}$ & 0.5906 & 0.5746 & 0.1194 & 0.1131 \\
\hline \multicolumn{5}{|l|}{ Panel B: Distress } \\
\hline & \multicolumn{2}{|c|}{ Interest Coverage } & \multicolumn{2}{|c|}{ Z-Score } \\
\hline & $(1)$ & $(2)$ & $(3)$ & $(4)$ \\
\hline $\operatorname{Breach}(0 / 1)$ & $\begin{array}{c}-1.3884^{* *} \\
(0.0337)\end{array}$ & & $\begin{array}{c}-2.5780^{*} \\
(0.0609)\end{array}$ & \\
\hline Severe Breach $(0 / 1)$ & & $\begin{array}{c}-2.1732^{* *} \\
(0.0201)\end{array}$ & & $\begin{array}{c}-4.1945^{* *} \\
(0.0473)\end{array}$ \\
\hline Low Breach $(0 / 1)$ & & $\begin{array}{c}-0.6319^{* *} \\
(0.0443)\end{array}$ & & $\begin{array}{l}-1.4145 \\
(0.1152)\end{array}$ \\
\hline Controls & Yes & Yes & Yes & Yes \\
\hline Year Fixed Effects & Yes & Yes & Yes & Yes \\
\hline Industry Fixed Effects & Yes & Yes & Yes & Yes \\
\hline Observations & 2,316 & 2,316 & 2,040 & 2,040 \\
\hline$R^{2}$ & 0.2681 & 0.2013 & 0.4506 & 0.3303 \\
\hline
\end{tabular}


Table 2.9: Conditional likelihood of future data breaches

This table presents probit regressions for the likelihood of a future data breach conditional on a breach occurring in year $t$ or $t-1$. The dependent variable is a dummy variable that takes a value of 1 if a firm experiences a data breach in year $t+1,0$ otherwise. Standard errors are clustered by firm, p-values are reported in parentheses with less than $1 \%, 5 \%$, and $10 \%$ levels of statistical significance denoted by $* * *, * *$, and $*$, respectively.

\begin{tabular}{lcccc}
\hline & $(1)$ & $(2)$ & $(3)$ & $(4)$ \\
\hline Breach $(0 / 1)_{t}$ & $0.4074^{* *}$ & & & 0.1834 \\
& $(0.0428)$ & & & $(0.3334)$ \\
Breach $(0 / 1)_{t-1}$ & & $0.5083^{* *}$ & $0.4285^{* *}$ & $0.4204^{*}$ \\
& & $(0.0186)$ & $(0.0475)$ & $(0.0529)$ \\
Year Fixed Effects & Yes & Yes & Yes & Yes \\
Industry Fixed Effects & No & No & Yes & Yes \\
Observations & 2,145 & 1,889 & 1,813 & 1,813 \\
Pseudo $R^{2}$ & 0.0105 & 0.0138 & 0.0305 & 0.0316 \\
\hline
\end{tabular}

Table 2.10: Robustness: CDS market reaction to data breaches

This table presents event-study results of the impact of data breaches on CDS spread. We report the CDS spread changes of the 15-day and 30-day cumulative abnormal returns (CARs) during an event window of $(-7,+7)$, and $(-15,+15)$ surrounding the data breach events. The standard deviations of the CDS spread changes are also presented in this table. We calculate CARs using both the market model and the market adjusted model. The adjusted CDS spread is the difference between the CDS spread and the benchmark index constructed as the cross-sectional average of all CDS spreads in the sample within the same credit rating category. ${ }^{* * *}, * *$, and $*$ denote significance at the $1 \%, 5 \%$, and $10 \%$ levels, respectively.

\begin{tabular}{lcc}
\hline & CAR $(-7,7)$ & CAR $(-15,15)$ \\
\hline Panel A: Market Model & & \\
\hline CAR (bps) & $4.2^{* * *}$ & $1.5^{* * *}$ \\
p-value & 0.000 & 0.000 \\
Standard Deviation & 20.9 & 24.6 \\
\hline Panel B: Market Adjusted Model & & \\
\hline CAR (bps) & $4.9^{* * *}$ & $0.79^{* * *}$ \\
p-value & 0.000 & 0.000 \\
Standard Deviation & 22.8 & 23.8 \\
\hline
\end{tabular}


Table 2.11: Robustness: Data breach and profitability

This table presents results about the relation between data breaches and firm profitability in the subsequent year. ROA is the dependent variable in Columns (1) and (2) and Sales is the dependent variable in Columns (3) and (4). The variable Breach(0/1) is set to one for breached firms in the year of the data breach, and zero otherwise; Severe Breach(0/1) is set to one for breaches in the top CDS spread change distribution (high CDS spread increase), and Low Breach(0/1) is set to one for breaches in the bottom of the CDS spread change distribution (low CDS spread increase). To limit the influence of outliers, we winsorize all continuous variables at the 1st and 99th percentiles. Standard errors are clustered by firm, p-values are reported in parentheses with less than $1 \%, 5 \%$, and $10 \%$ levels of statistical significance denoted by $* * *, * *$, and $*$, respectively.

\begin{tabular}{lcccc}
\hline & \multicolumn{2}{c}{ ROA $_{t+1}$} & \multicolumn{2}{c}{ Sales $_{t+1}$} \\
& $(1)$ & $(2)$ & $(3)$ & $(4)$ \\
\hline Breach $(0 / 1)$ & $-0.0023^{*}$ & & $-0.0024^{*}$ & \\
Severe Breach $(0 / 1)$ & $(0.0741)$ & & $(0.0653)$ & \\
& & $-0.0082^{*}$ & & $-0.0086^{* *}$ \\
Low Breach $(0 / 1)$ & & $(0.0791)$ & & $(0.0402)$ \\
& & 0.0037 & & $0.0043^{* *}$ \\
Controls & & $(0.5302)$ & & $(0.0242)$ \\
Year Fixed Effects & Yes & Yes & Yes & Yes \\
Industry Fixed Effects & Yes & Yes & Yes & Yes \\
Observations & 2,318 & 2,318 & 2,312 & Yes \\
$R^{2}$ & 0.4844 & 0.5175 & 0.2069 & 0.1131 \\
\hline
\end{tabular}


Table 2.12: Robustness: Interest coverage and the effect of data breaches on credit risk

This table presents results about the role of interest coverage in the relation between data breaches and credit risk. The dependent variable in Column 1 is the credit rating numerical variable and the dependent variable in Column 2 is CDS spread. The results in Column 1 is estimated by ordered logistic regression and by OLS regression in Column 2. The variable of interest in Columns 1-2 is Breach $(0 / 1) \times$ Interest Coverage. To limit the influence of outliers, we winsorize all continuous variables at the 1 st and 99th percentiles. Standard errors are clustered by firm, p-values are reported in parentheses with less than $1 \%, 5 \%$, and $10 \%$ levels of statistical significance denoted by $* * *, * *$, and $*$, respectively.

\begin{tabular}{lcc}
\hline & Credit Rating & CDS Spread \\
& $(1)$ & $(2)$ \\
\hline Breach(0/1) & $-0.0105^{*}$ & 7.4542 \\
Breach $(0 / 1) \times$ Interest Coverage & $0.0272^{* *}$ & $(0.6000)$ \\
& $(0.0122)$ & $\left(0.01833^{* *}\right.$ \\
Firm Size & $1.2483^{* * *}$ & $-43.9381^{* * *}$ \\
& $(0.0000)$ & $(0.0000)$ \\
Profitability & $5.4141^{*}$ & $-54.0141^{* *}$ \\
& $(0.0532)$ & $(0.0192)$ \\
Book Leverage & $-4.6941^{* * *}$ & $37.7929^{* * *}$ \\
& $(0.0000)$ & $(0.0000)$ \\
Asset Tangibility & 0.0552 & 34.1285 \\
& $(0.880)$ & $(0.3755)$ \\
Market-to-book & $0.9253^{* * *}$ & $-33.9221^{* *}$ \\
& $(0.0000)$ & $(0.0222)$ \\
Interest Coverage & $0.0197^{* * *}$ & $-3.3871^{*}$ \\
& $(0.0000)$ & $(0.0873)$ \\
Loss(0/1) & $-1.0111^{* * *}$ & $186.2599^{* * *}$ \\
& $(0.0000)$ & $(0.0000)$ \\
Capital Expenditure & 2.5015 & $-82.9718^{* *}$ \\
& $(0.4732)$ & $(0.0300)$ \\
Year Fixed Effects & Yes & Yes \\
Industry Fixed Effects & Yes & Yes \\
Observations & 1,993 & 2,318 \\
$R^{2}$ & 0.2754 & 0.3811 \\
\hline
\end{tabular}


Table 2.13: Variable definitions

This table provides the definition of the key variables used.

\begin{tabular}{|c|c|}
\hline Variable & "Definition \\
\hline $\operatorname{Breach}(0 / 1)$ & $\begin{array}{l}1 \text { for breached firms in the year of the data breach and } 0 \\
\text { otherwise }\end{array}$ \\
\hline Severe Breach $(0 / 1)$ & $\begin{array}{l}1 \text { breaches in the top CDS spread change distribution (high } \\
\text { CDS spread increase) and } 0 \text { otherwise }\end{array}$ \\
\hline Low Breach(0/1) & $\begin{array}{l}1 \text { for breaches in the bottom of the CDS spread change dis- } \\
\text { tribution (low CDS spread increase) and } 0 \text { otherwise }\end{array}$ \\
\hline Credit Rating & $\begin{array}{l}\text { Credit rating is a numerical score set to } 7 \text { for AAA ratings, } 6 \\
\text { for AA ratings, } 5 \text { for A ratings, } 4 \text { for BBB ratings, } 3 \text { for BB } \\
\text { ratings, } 2 \text { for } \mathrm{B} \text { ratings, and } 1 \text { for } \mathrm{C} \text { ratings }\end{array}$ \\
\hline CDS Spread & $\begin{array}{l}\text { Average annual five-year daily credit default swap spread with } \\
\text { modified restructuring clause, provided there are at least } 100 \\
\text { daily CDS spread }\end{array}$ \\
\hline Market-to-book & $\begin{array}{l}\text { Ratio of total book assets less the book value of common } \\
\text { equity plus the total market value of equity all divided by the } \\
\text { total book assets }\end{array}$ \\
\hline Firm Size & Natural logarithm of total book assets \\
\hline Profitability & $\begin{array}{l}\text { Ratio of annual income before extraordinary items to total } \\
\text { book assets }\end{array}$ \\
\hline Book Leverage & $\begin{array}{l}\text { Ratio of total book debt (short-term debt plus long-term debt) } \\
\text { to total book assets }\end{array}$ \\
\hline Asset Tangibility & $\begin{array}{l}\text { Ratio of gross property, plant, and equipment to total book } \\
\text { assets }\end{array}$ \\
\hline Market-to-book & $\begin{array}{l}\text { Ratio of total book assets less the book value of common } \\
\text { equity plus the total market value of equity all divided by the } \\
\text { total book assets }\end{array}$ \\
\hline Interest Coverage & $\begin{array}{l}\text { Ratio of annual operating income after depreciation and amor- } \\
\text { tization to annual interest expense }\end{array}$ \\
\hline Low Interest Coverage (0/1) & $\begin{array}{l}1 \text { if a firm belongs to the bottom of the interest coverage } \\
\text { distribution, and } 0 \text { otherwise }\end{array}$ \\
\hline $\operatorname{Loss}(0 / 1)$ & $\begin{array}{l}1 \text { if a firm reports loss in income before extraordinary items } \\
\text { and } 0 \text { otherwise }\end{array}$ \\
\hline Capital Expenditure & Ratio of capital expenditure to total book assets \\
\hline Sales Growth & Ratio of sales $_{t}$ to sales $_{t-1}$ \\
\hline Altman's Z & $\begin{array}{l}1.2(\text { working capital/total assets })+1.4 \text { (retained earn- } \\
\text { ings/total assets })+3.3(\text { earnings before interest and } \\
\text { taxes/total assets })+0.6(\text { market value of equity/book value } \\
\text { of long-term debt })+(\text { sale/total assets })\end{array}$ \\
\hline$\triangle C D S$ Spread & $\begin{array}{l}\text { Change in CDS spread computed for the time interval }[t-1 \text {, } \\
t]\end{array}$ \\
\hline$\triangle$ Adjusted CDS Spread & $\begin{array}{l}\text { Adjusted change in CDS spread calculated as the difference } \\
\text { between each firm's change in CDS return and the change in } \\
\text { the CDS index }\end{array}$ \\
\hline
\end{tabular}


Chapter 3

Does financing influence the sensitivity of cash and investment to asset tangibility? 


\subsection{Introduction}

A large body of theoretical and empirical literature shows that access to external financing is important for corporations, particularly for small firms. The literature on financial constraints provide evidence that financing frictions may impact the operating decisions of firms (Butler and Cornaggia (2011), Chava and Roberts (2008), and Whited (1992). Prior studies (Liberti and Mian (2010), Bernanke and Gertler (1989), and Kiyotaki and Moore (1997)) posit that contract incompleteness and limited enforceability reduce access to external finance, and that asset tangibility is fundamental to financial contracting and corporate financing capacity. This is because in default or bankruptcy states, the value that can be captured by creditors increases with asset tangibility. Therefore, by mitigating the extent of contractibility problems, asset tangibility increases the capability of firms to obtain external financing. Importantly, because investment generally relies on assetbased financing, tangible assets can increase investment when firms have imperfect access to credit.

Several previous studies also show that low tangible firms are typically exposed to costly external financing (Lei et al. (2018), Falato et al. (2013), and Lyandres and Palazzo (2016)). Because of the vulnerability of limited and expensive access to external finance, firms with low asset tangibility build up high cash balances to insure the availability of sufficient liquidity capable to weather adverse shocks. Low tangibility firms are financially constrained, and their investments and asset growth are more likely to be limited to available internal resources (Chava and Roberts (2008)). The tangibility of a firm's assets may impact its external financing capacity as well as its liquidity and investment strategy, and a negative asset tangibility sensitivity of cash could constrain firm growth if firms forgo investment to hold more cash.

Financial deregulation of public capital markets that improves access to external finance might remove binding financial constraints and moderate the dependence of cash and investment on asset tangibility, and promote firm growth. The theoretical underpin- 
ning of this line of inquiry is that capital market development expands the accessibility of alternative financing sources, and improves firms' access to lower cost external financing (Khurana et al. (2006)). Prior research provides some evidence consistent with this prediction. For instance, Krishnan et al. (2014) exploit the interstate banking deregulation to analyze how increased access to bank financing affects total factor productivity of firms. They find that greater access to bank financing increases firm total factor productivity, especially for financially constrained firms. Also, Demirgüç-Kunt and Maksimovic (1998) find that firm growth is positively associated with financial development. Love (2003) finds that financial development positively affects firm investment and Harford and Uysal (2014) show that access to debt markets affect corporate investment decisions.

Despite the far-reaching implications of asset tangibility on corporate cash holdings and investments, the impact of improved access to equity financing on the sensitivity of cash and investment to asset tangibility, particularly for smaller public firms has received little attention in the empirical finance literature. While a number of studies examine whether access to bank financing affect firm growth and investments (for example Krishnan et al. (2014); Kerr and Nanda (2009)), to the best of my knowledge, no study has directly explored the link between increased access to equity financing and the dependence of corporate cash holdings on asset tangibility. In this paper, I ask whether and how differential access to capital markets affects the dependence of cash and investment on asset tangibility.

A challenging hurdle facing empirical research in this area is the identification of an exogenous shock to a firm's access to equity markets to recognize differentials in the cost of external financing. Also, stock market development and corporate financial outcomes or firm growth could be endogenously determined, therefore, identifying the direction of causality is a major challenge. Little exists in the way of clearly exogenous variation for possible exploitation. Therefore, a conventional approach in the literature would examine a corporate financial or growth variable and a financial development proxy such as ratio of stock market traded value to Gross Domestic Product. However, such proxy could 
contain a market expectation of future growth, which could result in a spurious correlation between stock market development and the financial or growth variable (Levine and Zervos (1998)). Overcoming such a challenge requires a natural experiment setting where one can consider an exogenous shift in the availability of external financing. To bridge this gap, I provide evidence from a natural experiment created by the SEC 2008 financial deregulation that removed barriers to equity issuance and resulted in an exogenous and substantial decrease in the issuance cost (Gustafson and Iliev (2017)) for small listed firms. Consistent with prior literature (Gustafson and Iliev (2017)), I analyze the same set of firms before and after the exogenous shock to equity market access so as to avoid many of the challenges associated with endogenous priors. If the improved access to capital markets removes binding financial constraints on firms, I expect the sensitivity of cash and investment to asset tangibility to be dampened by the SEC equity issuance deregulation.

Prior to 2008, exchange-listed firms with public float or equity market capitalization of less than $\$ 75$ million were restricted from using shelf-registration to conduct accelerated SEOs. This restriction resulted in a different equity issuance environment for smaller and larger firms, and constituted a binding financial constraint that prevented about $25 \%$ of exchange-listed companies from raising follow-on equity capital using accelerated SEOs (Gustafson and Iliev (2017)). In 2008, the SEC adopted amendments to the eligibility requirements of shelf-registration (Form S-3). The 2008 deregulation, for the first time, allowed securities-exchange-listed firms with public float or equity market capitalization of less than $\$ 75$ million to conduct accelerated SEOs using shelf-registration. The aim of the amended public float requirement to shelf registration was to improve access to external finance and facilitate capital-raising efforts in the public equity markets by small listed firms. The SEC's decision to deregulate equity issuance using shelf registration plausibly provides an exogenous framework that allows for the study of how the exogenous shock differentially impacted the real outcomes of financially constrained and unconstrained firms. 
To carry out the empirical analysis, I follow Gustafson and Iliev (2017) and use the rule threshold of $\$ 75$ million and restrict the sample to firms with reported public floats between $\$ 10$ million and $\$ 150$ million, and I employ the difference-in-difference estimator to examine the effect of the deregulation of equity issuance on the sensitivity of cash and investment to asset tangibility. ${ }^{1}$ The advantage of using this public float restriction is that firms below and above the $\$ 75$ million threshold differ in terms of their access to shelf registration for accelerated SEOs but are unlikely to differ substantially in terms of other characteristics. In particular, firms with public float of less than $\$ 75$ million were ineligible for shelf registration, hence, financially constrained relative to firms with public float above the threshold. Therefore, employing this approach provides an assurance that the results are not driven by other potentially unobservable differences between the treated and control firms.

The results of this paper deliver a number of interesting findings. Consistent with the view that financing impacts corporate financial policies, I find that improved access to public capital markets lowers the asset tangibility sensitivity of cash. This suggests that as financial deregulation broadens the sources of corporate financing for constrained firms, it effectively reduces the sensitivity of cash to asset tangibility and moderates the need to hold cash among firms with low tangibility. While the methodology I employ provides a strong assurance that the baseline results are not simply driven by size differences or other potentially unobservable differences between the treated and control firms, I examine further whether public float size cutoffs other than the $\$ 75$ million have similar effects. I create two placebo groups, the lower placebo and upper placebo with cutoffs of $\$ 40$ million and $\$ 120$ million respectively. If the baseline results are due to size differences, then I expect these placebo cutoffs to have similar impact. The main results disappear in the upper and lower placebo tests. The results do not show differences in impact of the deregulation on the sensitivity of cash to asset tangibility. The non-results discount

\footnotetext{
${ }^{1}$ As robustness, I use a tighter band around the $\$ 75$ million threshold.
} 
the possibility that the baseline results are due to differential reaction of the treated and control firms.

Next, I examine the channels through which the equity issuance deregulation dampens the dependence of cash on asset tangibility. First, I check whether the moderating effect of the financial deregulation on the sensitivity of cash to asset tangibility is stronger in firms with high information asymmetry and find that it is indeed the case, consistent with the idea that high information asymmetry firms, which are typically financially constrained benefit more from increased access to external equity financing. This findings is not surprising because in an imperfect market, firms with high levels of information asymmetry face frictions in obtaining external capital, therefore, removing a binding constraint on their ability to obtain external financing should have stronger effect on them. This further supports the idea that financially constrained firms gain substantially more from greater access to financing as a result of the deregulation equity issuance. Second, I examine whether the equity issuance deregulation is more beneficial to firms with high-tech intensiveness. Firms with high levels of tech intensity are mostly reliant on external equity financing due to the intangibility nature of R\&D. Furthermore, the equity market imposes no collateral requirement and equity financing does not increase the probability of financial distress. Therefore, a deregulation that removes barriers to equity issuance and improves access to equity financing should be much more important for hightech intensive firms. Consistent with this expectation, I find that the equity issuance deregulation had a stronger dampening impact on the cash-tangibility sensitivity for firms with high-tech intensiveness. Overall, the findings suggest that young and high-tech intensive firms benefit more from a deregulation that removes barriers to equity issuance, which provides further support to the idea that greater access to equity financing dampens the dependence of cash on asset tangibility by relieving firms of financial constraints.

Prior papers (such as Brown and Petersen (2009); Şenay Agca and Mozumdar (2008); Andren and Jankensgard (2015)) document a decreasing investment-cash flow sensitivity and posit that the rising importance and development of U.S. public equity markets as a 
source of financing, particularly for young small firms mainly drives this trend. I examine whether the deregulation of equity issuance, which gave small firms increased access to equity financing impacted the investment-cash flow sensitivity of the treated firms relative to the control firms. If the deregulation indeed removed a binding financial constraint of small firms, I expect the investment-cash flow sensitivity to decrease following the deregulation. I estimate the investment-cash flow sensitivity, and find that the equity issuance deregulation had a negative impact on the investment-cash flow sensitivity. This provides evidence that an improvement in the functioning of capital markets could reduce the financing constraints of firms. This will promote easier access to external funds for small firms with good investment opportunities and in turn, the improvement in capital allocation will enhance firm growth.

An important implication of the findings is that by lessening the impact of tangible assets on cash holdings, financial deregulation could permit low tangibility firms to hold less cash and undertake more investment opportunities. In line with this prediction, I find evidence that access to public capital markets has real effect on the ability to make investments. Specifically, I find that the financial deregulation of equity issuance dampened the positive impact of asset tangibility on investment. The implication of this is that, following the deregulation, small firms have improved access to additional financing source, hence, reserve less cash, and are able to increase their investments. This result supports the hypothesis that improved access to external financing may increase constrained firms' access to additional profitable and productive projects that they may otherwise not be able to pursue.

While the deregulation of equity issuance improved access to external finance, which can be valuable to the size of corporate investments, it does not automatically translate into good investment decisions. A natural follow-up question is whether the improved access to cheaper equity financing following the equity issuance deregulation is dissipated by the treated firms taking on unproductive or less productive projects or whether this increases the ability of firms to undertake additional profitable projects that they were 
unable to take on prior to the equity issuance deregulation. I extend the analysis further to examine the effect of the equity issuance deregulation on the quality of investment decisions as proxied by investment efficiency. This analysis is important because more is not necessarily better in the case of corporate investments. With this approach, rather than looking at the size of firm investments, I focus directly on the quality of investment decisions as gauged by firms' investment efficiency gains. The measure of investment efficiency captures the difference between expected and actual investment levels. Therefore, gains in investment efficiency cannot merely be the result of an increase in the size of corporate investments or scale of operations, but rather the result of increased access to additional profitable projects that allows a firm to become more efficient. In this situation, I expect the increased access to equity financing to lead to the highest increase in investment efficiency for those firms that were initially financially constrained due to the limitation on the use of shelf-registration. I find that investment efficiency for the treated firms increased following the deregulation of equity issuance. This suggests that the deregulation by facilitating access to external finance, permitted small firms to finance prudent and productive investments that otherwise might not be pursued.

Finally, I examine the effect of the deregulation on the asset side of the firms' balance sheet to shed light on the implication of the deregulation for firm growth. I find that the treated firms increase their growth of fixed assets, suggesting that a deregulation that improves access to external equity finance promotes faster firm growth. This provides evidence that public firms in a developed financial market like the U.S. benefit from a financial deregulation that removes barriers to external equity financing.

This paper delivers important findings and contributions. First, it brings evidence from a natural experiment to the literature on the role of tangible assets in determining corporate financial and investment policies. As far as I am aware, it is the first study that examines the causal effect of financing on the sensitivity of cash and investment to asset tangibility at the firm level, with the objective to identify the causal impact of financial development on corporate investment policy. It is related to the recent emerging 
literature on the role of tangibility on financial and investment policies of firms. Chaney et al. (2012) examine the sensitivity of investment to collateral and provide evidence that shocks to collateral have significant impact on the investment decisions of firms. Gan (2007) provide evidence from the Japan real estate bubble that negative shocks to the assets of top lenders affects the investment and market valuations of firms.

Second, to the best of my knowledge, no previous study has investigated the influence of improved access to equity capital on the sensitivity of cash and investment to asset tangibility before and after an exogenous and substantial shock to external equity financing; this paper helps fill this gap. Liberti and Mian (2010) show that financial development reduces the collateralization rate between high-and low-risk borrowers. Love (2003) shows that financial development affects growth by lessening financial constraints that would otherwise distort efficient allocation of investment. Harford and Uysal (2014) examine the effect of differential access to debt markets on investment, and finds that improved access to debt markets increases the likelihood to undertake acquisitions. This paper is closest in spirit to Lei et al. (2018) as they conduct a cross-country study of the impact of financial development on the cash-asset tangibility sensitivity. However, I provide a firm-level evidence that the deregulation of capital markets reduces firms' financing constraints, and allows for improved access to external finance for the funding of profitable investments, which enhances firm growth. While Lei et al. (2018) conventionally uses a macro level financial development proxy such as private credit to GDP, I use a plausibly natural experiment framework to capture the magnitude of the causal effect of financing on the cash-asset tangibility sensitivity at the firm-level, and employ a testing procedure that resolves the problem of endogeneity between financial development and financial variables. Financial development proxy such as private credit to GDP as used in Lei et al. (2018) and corporate financial outcomes could be endogenously determined (Levine and Zervos (1998)). The economic forces that promote financial development could also affect the interplay between cash holdings and asset tangibility. 
Third, this paper is related to the literature on financing constraints and investment decisions. Consistent with Love (2003), I show that financial deregulation lessens the financial constraints of firms. This allows for easier access to external funds, which consequently lowers the dependence of cash and investment on tangibility, and the improvement in capital allocation promotes firm growth. Also, I bring evidence from a natural experiment framework to the sensitivity of investment to cash flow literature. Recent papers (Brown and Petersen (2009), and Şenay Agca and Mozumdar (2008)) find a decreasing investment-cash flow sensitivity over time and attribute the declining sensitivity to capital market developments. Furthermore, I document that financial deregulation by reducing the sensitivity of cash and investment to asset tangibility promotes investment efficiency by ensuring efficient allocation of resources, which enables firms to undertake profitable projects they would otherwise forgo. This is very vital from a policy perspective if the objective is to promote the growth of small listed firms. A related strand of literature examines the relationship between access to finance and productivity. Krishnan et al. (2014) show that improved access to bank financing enhances productivity of small and young firms. Butler and Cornaggia (2011) examine how producers adapt their productivity in the midst of varying levels of access to finance, and find that producers increase their production in times of strong access to finance. I contribute to this strand of literature by showing that the improved access to equity financing reduces the dependence of cash and investment on tangible assets, and enables firms to finance productivity-enhancing investments.

Overall, the findings of this paper show that even in a highly developed market such as the U.S., access to external equity financing is an important component of corporate behaviour. This provides micro-level evidence that sheds new light on the role of financial deregulation in fostering economic growth.

The remainder of the paper is structured as follows. Section 3.2 provides institutional background information in relation to the financial deregulation. Section 3.3 describes 
the sample construction. Section 3.4 and Section 3.5 discuss the empirical approach and results, respectively. Section 3.6 concludes the paper.

\subsection{Institutional detail: deregulation of equity is- suance in the US}

Accelerated SEOs have become an increasingly common and popular method of raising seasoned public equity. Previous studies (Gao and Ritter (2010) and Bortolotti et al. (2008)) document that accelerated deals are faster and cheaper than the traditional fully marketed offerings, hence, their popularity. Accelerated SEO proceeds accounted for more than half the total value of SEOs in the US in 2004 (Bortolotti et al. (2008)). In the US, accelerated SEOs are usually conducted through shelf registrations. The SEC, in 1982, introduced shelf registration, which permits firms to pre-file estimated offerings that they reasonably anticipate issuing in the future. The shelf registered offerings can be subsequently issued in full or in part. According to the SEC, the ability to pre-file expected offerings using Form S-3 (shelf registration) confers significant benefits on eligible firms. SEOs issued off the shelf allow firms to avoid regulatory delays and interruptions, and minimize the costs associated with the SEO process. ${ }^{2}$ By having control over the timing of issuing the shelf-registered securities, firms are able to raise capital on more favourable terms. Consequently, the ability to issue securities off the shelf as needed provides firms with a significant financing alternative to other funding methods. ${ }^{3}$ As at 2003, more than two-thirds of firms eligible to use Form S-3 had issued SEOs off the shelf (Kumar and Shome (2008)).

However, prior to the 2008 deregulation, which amended the eligibility requirement for using shelf registration, public firms conducted off the shelf equity offerings only if their public float was $\$ 75$ million or more. This restriction resulted in different equity

\footnotetext{
${ }^{2}$ See the SEC release NO. 33-8878

${ }^{3}$ See the SEC release NO. 33-8878
} 
issuance environment for smaller and larger firms. It effectively prevented about $25 \%$ of exchange listed companies (mainly small firms) from raising follow-on equity capital using accelerated SEOs (Gustafson and Iliev (2017)). The final text of the 2008 amendment to the eligibility requirement for Form S-3 (shelf registration) emphasizes how the restriction prevented a large number of listed firms from conducting accelerated SEOs. The final SEC text states that "These amendments are intended to allow a large number of public companies to benefit from greater flexibility and efficiency in accessing the public securities markets afforded by Form S-3."

By amending the $\$ 75$ million public float eligibility requirement, the SEC, argues that smaller companies will have greater financing flexibility, efficiency, and an enhanced access to capital in the equity markets, with less cost and burden. Because smaller firms have fewer financing options relative to their larger counterparts, removing public float limitations to shelf registration should facilitate their capital-raising efforts in the public equity markets. Consequently, Gustafson and Iliev (2017) find that, post-deregulation; the treated firms significantly increased their public equity capital issuance using shelf registration, and importantly, the issuance costs for off the shelf accelerated SEOs significantly declined.

\subsection{Sample construction}

To construct the sample, I begin with exchange listed firms with public float between $\$ 10$ million and $\$ 150$ million covering the period 2002 and 2013. The public float data was made available by Gustafson and Iliev (2017). ${ }^{4}$ I restrict the public float data to between $\$ 10$ million and $\$ 150$ million because of the 2008 deregulation, which permitted firms with public float of less than $\$ 75$ million to use shelf registrations to raise equity capital. Also, given that the requirement for public companies to report their public float in $10-\mathrm{K}$ filings came into effect in 2002, I restrict the sample period to begin from 2002. Because

\footnotetext{
${ }^{4} \mathrm{~A}$ detailed description of the process of extracting the public float data can be found in their paper.
} 
firms with public float between $\$ 70$ million and $\$ 80$ million can change treatment status during the year, I follow Gustafson and Iliev (2017) and exclude such observations from the dataset. This ensures treated firms have public float between $\$ 10$ million and $\$ 70$ million, and control firms have public float between $\$ 80$ million and $\$ 150$ million. I then merge the public float data with the relevant annual firm-level accounting data from the Compustat database.

I follow previous literature (Almeida et al. (2004), Opler et al. (1999), and Hoberg et al. (2014)) and exclude firms in the financial (SIC codes 6000 - 6999) and utilities (SIC codes 4900 - 4950) industries. I exclude shell companies and firms that are not registered on national stock exchanges because the deregulation is not applicable to them. I also exclude firm-year observations that have missing and negative data for total assets. I further drop firms with negative cash. While I account for general trends in the empirical setting, the onset of the global financial crisis in 2008 presented a major economic downturn and negative shock to access to external financing. To ensure the analysis of the paper is not driven by this systemic event, I follow Gustafson and Iliev (2017) and account for the effect of the financial crisis by excluding firm-year observations for which more than six months fall within the financial crisis as defined by the National Bureau of Economic Research. ${ }^{5}$ The final sample consists of 7,213 firm year observations.

Table 3.1 reports descriptive statistics for the main firm characteristics split by firm treatment status in the pre and post shelf registration rule period. All data filters described earlier such as excluding firms with negative or missing assets and cash, excluding financial crisis period, and all continuous variables winsorized at the $1 \%$ and $99 \%$ in both tails are applied to the descriptive statistics. As can be seen in Table 3.1, the treated firms are smaller in terms of public float and size relative to the control firms, but they are much comparable in terms of other firm characteristics. This suggests that the untreated firms serve as suitable control group to investigate the effect on the equity issuance deregulation on the dependence of cash and investment on asset tangibility.

\footnotetext{
${ }^{5}$ Following Gustafson and Iliev (2017), observations with fiscal year-end from June 2008 to December 2009 are excluded as they fall within the NBER financial crisis definition of December 2007 to June 2009.
} 


\subsection{Empirical strategy}

This section provides a description of our regression specification. I use the difference-indifferences approach to formally explore the influence of the 2008 equity issuance deregulation on the sensitivities of cash and investment to asset tangibility. With this empirical strategy, I exploit the fact that the treated and untreated firms faced different regulatory requirements before the deregulation, but both are in the same regulatory environment following the amendment to the eligibility requirement. This difference-in-difference strategy allows for a better isolation of the independent role of financial deregulation on corporate financial policies. In the spirit of Gustafson and Iliev (2017), I specify the following model.

$$
\begin{aligned}
Y_{i, t}= & \alpha+\beta_{1} \text { Asset Tangibility } y_{i, t}+\beta_{2} \text { Asset } \text { Tangibility }_{i, t} \times \operatorname{Treated}(0 / 1)_{i, t} \times \operatorname{Post}(0 / 1)_{t} \\
& +\beta_{3} \operatorname{Treated}(0 / 1)_{i, t} \times \operatorname{Post}(0 / 1)_{t}+\beta_{4} \operatorname{Treated}(0 / 1)_{i, t}+\gamma X_{i, t}+\delta_{t}+\rho_{j}+\varepsilon_{i, t},
\end{aligned}
$$

where $i, j$, and $t$ index firm, industry and year respectively. $Y_{i, t}$ represents the dependent variables. Asset Tangibility $_{i, t}$ is the ratio of net property, plant, and equipment (PPENT) to total book assets $(\mathrm{AT})$. Treated $(0 / 1)_{i, t}$ is a dummy variable set to one for firms with public float of less than $\$ 75$ million, otherwise zero. Post $(0 / 1)_{t}$ switches to one for periods after 2008, indicative of the years after the deregulation. $X_{i, t}$ is a set of firm-level control variables. $\delta_{t}$ is year fixed effects, and it captures shocks that might affect the outcome variables. $\rho_{j}$ controls for industry fixed effects. Standard errors are clustered at the firm level to correct for serial correlation. Because the effect of a separate Post $(0 / 1)_{t}$ term is subsumed by the year fixed effects $\left(\delta_{t}\right)$, I exclude it from the specification.

$\beta_{1}$ measures the direct effect of tangibility on cash and investment. Because firms with high level of tangibility have greater access to debt financing as they can collateralize their assets, they have less incentive to hold cash, hence the expectation is that Asset 
Tangibility should have a negative marginal effect on Cash. I am most interested in the coefficient estimate $\beta_{2}$ of the triple interaction term Asset Tangibility ${ }_{i, t} \times{\text { Treated }(0 / 1)_{i, t}}$ $\times \operatorname{Post}(0 / 1)_{t}$. A positive $\beta_{2}$ estimate would imply that financing in the form of stock issuance deregulation dampens the negative sensitivity of Cash to Asset Tangibility. By reducing the effect of asset tangibility on cash, the deregulation should promote investment by low tangibility firms.

To examine the impact of the financial deregulation on the sensitivity of investment to cash flow, I follow Andren and Jankensgard (2015) and estimate the following model.

$$
\begin{aligned}
Y_{i, t}= & \alpha+\beta_{1} \text { Cash Flow } \text { Fl, }+\beta_{2} \text { Cash Flow } \text { Fi,t } \times \operatorname{Treated}(0 / 1)_{i, t} \times \operatorname{Post}(0 / 1)_{t} \\
& +\beta_{3} \operatorname{Treated}(0 / 1)_{i, t} \times \operatorname{Post}(0 / 1)_{t}+\beta_{4} \operatorname{Treated}(0 / 1)_{i, t}+\gamma X_{i, t}+\delta_{t}+\rho_{j}+\varepsilon_{i, t},
\end{aligned}
$$

where $Y_{i, t}$ represents investment, measured by capital expenditure scaled by beginning year total book assets. Cash Flow $w_{i, t}$ represents firm internal cash flow. $X_{i, t}$ represents firm-level controls for Market-to-book, which proxies for investment opportunities, Cash, and Book Leverage. The coefficient $\beta_{1}$ is investment-cash flow sensitivity and the coefficient $\beta_{2}$ captures the impact of the financial deregulation on the sensitivity of investment to cash flow. All other variables remain the same as defined in Eq.(3.1).

Also, to examine the effect of the financial deregulation on investment efficiency, I estimate the following model.

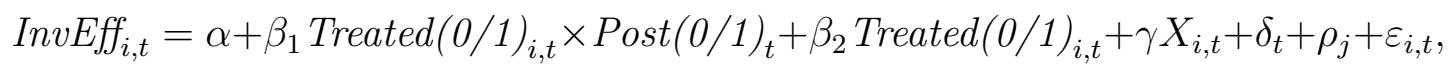

where $\operatorname{InvEff} f_{i, t}$ represents investment efficiency, and all other variables remain the same as defined in Eq.(3.1).

Conceptually, investment efficiency implies firms undertaking all positive net present value projects. Biddle et al. (2009) estimates a model of investment in terms of growth 
opportunities. Investment efficiency exists when the residuals from the investment model equals to zero, suggesting there is no deviation from the expected level of investment. Positive deviations from the expected level of investment imply that firms are overinvesting while negative deviations or residuals imply firms do not undertake all positive net present value projects. To examine how the deregulation of equity issuance affected firm investment efficiency, I follow Biddle et al. (2009) and estimate a model that predicts firm investment level based on growth opportunities using sales growth, and alternatively Tobin's Q or both. Deviations from the model capture investment inefficiency.

$$
\text { Investment }_{i, t}=\alpha+\beta_{1} X_{i, t}+\varepsilon_{i, t}
$$

where $X_{i, t}$ is either Sales Growth or Tobin's $Q .{ }^{6}$ I estimate Eq.(3.4) cross-sectionally for each industry-year based on Fama and French (1997) industry classification. Deviations as reflected in the residuals, capture the deviations from the expected investment level. The residuals proxy for investment inefficiency. Again, a positive residual implies that a firm is investing at a rate higher than the expected level based on sales growth or Tobin's Q, representing overinvestment. However, a negative residual means that a firm's real investment is less than the expected level, indicating a case of underinvestment. To obtain investment efficiency I multiply the absolute value of the deviations (residuals) by -1. Therefore, a higher value represents a higher investment efficiency.

\subsection{Empirical analysis}

\subsubsection{Financing, asset tangibility, and cash}

Table 3.2 presents the estimation results of Eq.(3.1). Columns 1 and 2 report the baseline estimation results. In the baseline estimation, I restrict the sample to firms with public float between $\$ 10$ million and $\$ 150$ million with the aim of balancing the need for

\footnotetext{
${ }^{6}$ Sales Growth is the change in sales from period $t-1$ to $t$
} 
statistical power with the assumption that the treated and control firms are similar. In Columns 3 and 4, I test whether the baseline results hold to a tighter band of public float between $\$ 25$ million and $\$ 125$ million. This tighter band selection should inherently reduce statistical power to obtain conservative statistical inferences. In order to examine the impact of the increased access to financing on the dependence of cash on asset tangibility within a short horizon around the public float rule change, in Columns 5 and 6, I restrict the sample for the cash-tangibility sensitivity regression to the time period within the five years of the equity issuance deregulation. ${ }^{7}$

In Columns 1, 3 and 5, I measure the direct sensitivity of cash to asset tangibility. Across all the columns, the coefficient estimate of Asset Tangibility is negative and statistically significant, suggesting that high asset tangibility leads to lower cash holdings. Firms with high asset tangibility have greater access to financing as they can collateralize their assets, hence, have lower need to hold high cash balances. In Columns 2, 4 and $6, \beta_{2}$, the coefficient of the triple interaction Asset Tangibility $y_{i, t} \times \operatorname{Treated}_{(0 / 1)_{i, t}}$ $\times \operatorname{Post}(0 / 1)_{t}$, which is the interaction of interest is positive and statistically significant across all columns. This implies that the negative relation between Asset Tangibility and Cash is dampened by financing. The financial deregulation by removing a binding financial constraint on treated firms (small firms), moderates the asset tangibility sensitivity of cash.

In Table 3.3, I perform a placebo test to ensure that the results in Table 3.2 are not due to size differences between the treated and control firms. I examine whether public float size cutoffs other than the $\$ 75$ million have similar effects. If the baseline results are due to size differences between the treated and untreated firms, then the alternative size cutoffs would have a similar effect. I create two placebo groups, the lower placebo and upper placebo. The lower placebo consists of firms with public float from $\$ 10$ million to $\$ 70$ million with a cutoff of $\$ 40$ million, so Treated $(0 / 1)$ is now set to one for a firm with a public float of less than $\$ 40$ million, otherwise zero. In the upper placebo, firms

\footnotetext{
${ }^{7}$ Here, the sample period is from 2005 to 2012 but excludes the financial crisis period of 2008 and 2009 as described in the earlier section. All the data filters are still included in this shorter sample period
} 
have public float from $\$ 90$ million to $\$ 150$ million with a cutoff of $\$ 120$ million. Again, Treated(0/1) is now set to one for a firm with a public float of less than $\$ 120$ million, otherwise zero. The results as presented in Table 3.3 are different from the baseline results in Table 3.2. The coefficients of the triple interaction of interest Asset Tangibility $\times \operatorname{Treated}(0 / 1) \times \operatorname{Post}(0 / 1)$ become insignificant for both the lower and upper placebo groups. This discounts the possibility that the baseline results are due to differential reaction of the treated and control firms.

\subsubsection{Information asymmetry and tech intensiveness}

The evidence so far suggests that the deregulation of equity issuance dampens the impact of asset tangibility on cash balances. In this section, I examine in detail the channels through which the deregulation influences the dependence of cash on asset tangibility.

First, I examine whether the deregulation is particularly beneficial to firms with high levels of information asymmetry. In an imperfect market, firms with high levels of information asymmetry face frictions in obtaining external capital (Armstrong et al. (2011), and Easley and Maureen (2004)). They face high financial constraints as they are unable to undertake profitable projects in the absence of adequate financing. However, since the equity issuance deregulation lead to an exogenous increase in the availability of financing, I anticipate firms with high information asymmetry to benefit more from the deregulation. To examine the differential impact of the deregulation on the level of firm information asymmetry, I sort the sample of firms into low and high information asymmetry firms based on firm age. I measure firm age as the number years since a firm first appeared in Compustat. For each year, I rank the firms using the firm age over the sample period and categorize firms in the top (bottom) of the distribution as the low (high) information asymmetry group. As indicated earlier, I expect the deregulation to be more important for firms with high information asymmetry. That is, the deregulation should have a higher impact on the sensitivity of cash to asset tangibility for the high information asymmetry group than for the low information asymmetry group. 
Next, I test whether the improved access to stock market financing is particularly important for high tech intensive firms. Here, the idea is that the intangibility nature of R\&D limits the ability of firms to use debt finance. The equity market imposes no collateral requirement and equity financing does not increase the probability of financial distress. Therefore, a deregulation that removes barriers to equity issuance and improves access to equity financing should be much more important for high-tech intensive firms. Again, I expect the deregulation to have a stronger impact on the sensitivity of cash to asset tangibility for firms with high tech intensiveness. I follow Hsu et al. (2014) and measure a firm's tech intensiveness as the annual gross growth in R\&D expenditure. For each year, I rank the firms and categorize those with tech intensiveness in the top (bottom) of the distribution as the high (low) tech intensiveness group.

Table 3.4 presents the results. As anticipated, the equity issuance deregulation had a stronger dampening impact on the cash-tangibility sensitivity for firms with high information asymmetry and those with high-tech intensiveness. Across Columns 1-4, the coefficient estimate of Asset Tangibility is negative and statistically significant. However, the coefficient estimate of the triple interaction Asset Tangibility $\times$ Treated $(0 / 1) \times$ Post(0/1) is positive and significant for young and high-tech intensiveness group. This suggests that young and high-tech intensive firms benefit more from a deregulation that removes barriers to equity issuance.

\subsubsection{Financing and the investment-cash flow sensitivity}

In a seminal paper, Fazzari et al. (1988) find that when firms face binding financial constraints, they display a greater sensitivity of investment to cash flow. Put simply, for financially constrained firms, investment varies with the availability of internal cash flow, rather than with the availability of profitable projects. A number of recent research papers document a decreasing investment-cash flow sensitivity (Brown and Petersen (2009), Şenay Agca and Mozumdar (2008), and Andren and Jankensgard (2015)) and posit that the rising importance and development of public equity markets as a source of financing, 
particularly for young small firms is the reason for this trend. In this section, I examine whether the removal of barriers to equity issuance and the reduction in the cost of external financing impacted the investment-cash flow sensitivity of the treated firms relative to the control firms. If the deregulation indeed removed a binding financial constraint of small firms, then I expect the sensitivity to decrease following the deregulation. All other things being equal, the treated firms who gained improved access to public capital markets following the deregulation should increase the use of external finance and increase the rate of investment. Specifically, the deregulation of equity issuance should lower the investment-cash flow sensitivity for treated firms relative to the control firms.

Table 3.5 presents the estimation results of investment-cash flow sensitivity as modeled in Eq.(3.2). In Column 1, I measure the straightforward investment-cash flow sensitivity for the entire sample. The Cash Flow coefficient is positive and statistically significant at the $1 \%$ level, suggesting a positive relation between investments and internal cash flow. In Column 2, I formally test whether the investment-cash flow sensitivity is affected by the improved access to external financing following the deregulation. Here, the variable of interest is $\beta_{2}$ of the triple interaction Cash Flow $i, t \times \operatorname{Treated}(0 / 1)_{i, t} \times \operatorname{Post}(0 / 1)_{t}$. As can be seen in Column 2, the coefficient of the variable of interest is negative and statistically significant at the $1 \%$ level. This result is consistent with the the interpretation that the treated firms were financially constrained before the deregulation but experienced a relaxation of the constraint following the deregulation. This suggests that improved access to external equity finance has important influence on the investment-cash flow sensitivity.

\subsubsection{Financing, asset tangibility, and investment}

So far, the findings show that deregulation allows low-tangibility firms to hold less cash. By dampening the impact of asset tangibility on cash balances, the deregulation allows low-tangibility firms to reserve less cash and could potentially enable them undertake more investment opportunities when they do arise. I investigate this implication by 
examining the impact of the deregulation on the asset tangibility sensitivity of investment. Table 3.6 presents the estimation results of Eq.(3.1) but with investment as the dependent variable.

In Column 1, I examine the direct effect of the deregulation on investment. The coefficient estimate of Treated $(0 / 1) \times \operatorname{Post}(0 / 1)\left(\beta_{3}\right)$ is positive and significant, which indicates that investment for the treated firms relative to the untreated firms, increased following the deregulation. Small firms responded to the improved access to external equity financing by significantly increasing investment spending. This is consistent with the findings in Gustafson and Iliev (2017), that the improved access to shelf registration and the accompanying reduction in issuance costs lead to more investment. In Column 2, I examine the direct effect of asset tangibility on investment. The coefficient estimate of Asset Tangibility is positive and statistically significant. This indicates that the investment policy of a firm is related to its level of tangibility. The positive sensitivity of investment to asset tangibility is consistent with the findings in Chaney et al. (2012) that investment increase by $\$ 0.06$ with each $\$ 1$ increase in collateral value. In Column 3 , I examine the baseline estimate of Eq.(3.1) to assess the role of the deregulation. I find that $\beta_{2}$, the coefficient of the triple interaction Asset Tangibility $_{i, t} \times \operatorname{Treated}_{(0 / 1)_{i, t}} \times$ $\operatorname{Post}(0 / 1)_{t}$, is negative and statistically significant, which indicates that equity issuance deregulation reduces the impact of asset tangibility on investment. Following the equity issuance deregulation, the treated firms have better or improved access to external finance, and hence have less incentive to accumulate cash. Therefore, by reserving less cash, the treated firms are able to make more capital investments.

\subsubsection{Financing and the quality of investment decisions}

The previous section examines the effect of the equity issuance deregulation on the size of investment. However, more may not necessarily be better in the case of investment. So in this section, I examine the effect of the equity issuance deregulation on the quality of investment decisions as gauged by efficiency gains of the treated firms relative to the 
control sample. A detailed description of the estimation of the investment efficiency measure is described in Section 3.4.

The results are presented in Table 3.7. The dependent variable in all columns is investment efficiency. The investment deviations from the investment model of Eq.(3.4) are based on sales growth in Column 1, Tobin's Q in Column 2, and both sales growth and Tobin's Q in Column 3. The measure of investment efficiency captures the difference between expected and actual investment levels. Therefore, gains in investment efficiency cannot merely be the result of an increase in the size of corporate investments. Across all columns, I find that the coefficients associated with the variable of interest, Treated $(0 / 1) \times \operatorname{Post}(0 / 1)$, are all positive and highly statistically significant, suggesting that the deregulation by facilitating access to external finance, permitted small firms to finance prudent and productive investments that otherwise might not have been pursued.

\subsubsection{Financing and firm asset growth}

The findings so far show that equity issuance deregulation permits low tangibility firms to hold less cash and invest more. The deregulation by improving access to external equity finance, moderates the asset tangibility sensitivity of cash, and enables firms to make more investment. I extend the analysis further to examine the direct financing channel by which the deregulation increased corporate investment. I investigate this by looking at the asset side of the firms' balance sheet to shed light on the implications of the deregulation for firm growth. If the increase in capital investment is due to increased fixed asset growth, I expect an increase in the growth of fixed asset following the deregulation.

In Table 3.8, I document how the book value of long-term (fixed) assets changed around the deregulation. I estimate a specification similar to Eq.(3.1) but with fixed assets growth as the dependent variable. In order, Columns 1-4 report regression results for longterm (fixed) assets growth, natural logarithm of long-term (fixed) assets growth, net fixed assets growth, and natural logarithm of net fixed assets growth. Across specifications 1-4, the coefficients associated with the variable of interest, Treated $(0 / 1) \times \operatorname{Post}(0 / 1)$, are all 
positive and statistically significant at the $1 \%$ level, suggesting growth in the proportion of fixed assets following the equity issuance deregulation. This is consistent with the findings in Tang (2009), who finds that credit rating refinement upgrades improves credit market access and promotes faster asset growth. Overall, the findings suggest that equity issuance deregulation improves firms' access to external financing, which in turn enables them to increase their capital investments by providing funds for more fixed asset investment.

\subsubsection{Robustness test}

As a robustness check, I re-estimate the baseline equation with the inclusion of firm fixed effects. The results are presented in Table 3.9. In Columns 1 and 2, the dependent variable is cash, and the dependent variable in Columns 3 and 4 is investment. Columns 1 and 3 report regression estimates of the direct sensitivity of cash and investment to asset tangibility, respectively. In Columns 2 and 4 , Asset Tangibility $\times$ Treated $(0 / 1) \times$ Post(0/1) tests for the impact of deregulation on the sensitivities of cash and investment to asset tangibility. In Column 1, the coefficient estimate of Asset Tangibility is negative and statistically significant, suggesting that high asset tangibility leads to lower cash holdings. In Column 2, the coefficient of the triple interaction Asset Tangibility T $_{i, t}$ $\times$ Treated $(0 / 1)_{i, t} \times \operatorname{Post}(0 / 1)_{t}$, which is the interaction of interest is positive and statistically significant. This implies that the negative relation between Asset Tangibility and Cash is dampened by financing.

In Column 3, the coefficient estimate of Asset Tangibility is positive and statistically significant. This indicates that the investment policy of a firm is positively related to its level of tangibility. In Column 4, the coefficient of the triple interaction Asset Tangibility $\times \operatorname{Treated}(0 / 1)_{i, t} \times \operatorname{Post}(0 / 1)_{t}$, is negative and statistically significant, which indicates that deregulation reduces the impact of asset tangibility on investment. 


\subsection{Conclusion}

This paper examines the impact of equity issuance deregulation on the sensitivity of cash and investment to asset tangibility. I use a natural experiment created by the financial deregulation of seasoned equity issuance in the U.S. to investigate the influence of financing on the dependence of cash and investment on asset tangibility. I show that financial deregulation dampens the sensitivity of cash to asset tangibility, and promotes investment and firm growth. This provides evidence that public firms in well developed financial markets such as the U.S. benefit from a financial deregulation that removes barriers to external equity financing, shedding light on the role of financial markets in fostering growth. 


\section{Bibliography}

Almeida, H., Campello, M., and Weisbach, M. S. (2004). The cash flow sensitivity of cash. The Journal of Finance, 59(4):1777-1804.

Andren, N. and Jankensgard, H. (2015). Wall of cash: The investment-cash flow sensitivity when capital becomes abundant. Journal of Banking $\&$ Finance, 50:204-213.

Armstrong, C. S., Core, J. E., Taylor, D. J., and Verrecchia, R. E. (2011). When does information asymmetry affect the cost of capital? Journal of Accounting Research, 49(1):1-40.

Bernanke, B. and Gertler, M. (1989). Agency costs, net worth, and business fluctuations. American Economic Review, 79:14-31.

Biddle, G. C., Hilary, G., and Verdi, R. S. (2009). How does financial reporting quality relate to investment efficiency? Journal of accounting and economics, 48:112-131.

Bortolotti, B., Megginson, W., and Smart, S. B. (2008). The rise of accelerated seasoned equity underwritings. Journal of Applied Corporate Finance, 20(3):35-57.

Brown, J. R. and Petersen, B. C. (2009). Why has the investment-cash flow sensitivity declined so sharply? rising $\mathrm{r} \& d$ and equity market developments. Journal of Banking \& Finance, 33(5):971-984.

Butler, A. W. and Cornaggia, J. (2011). Does access to external finance improve productivity? evidence from a natural experiment. Journal of Financial Economics, 99(1):184-203.

Chaney, T., Sraer, D., and Thesmar, D. (2012). The collateral channel: How real estate shocks affect corporate investment. American Economic Review, 102(6):2381-2409.

Chava, S. and Roberts, M. R. (2008). How does financing impact investment? the role of debt covenants. The journal of finance, 63(5):2085-2121.

Demirgüç-Kunt, A. and Maksimovic, V. (1998). Law, finance, and firm growth. The Journal of Finance, 53(6):2107-2137.

Easley, D. and Maureen, O. (2004). Information and the cost of capital. The journal of finance, 59(4):1553-1583.

Şenay Agca and Mozumdar, A. (2008). The impact of capital market imperfections on investment-cash flow sensitivity. Journal of Banking \&f Finance, 32(2):207-216. 
Falato, A., Kadyrzhanova, D., and Sim, J. (2013). Rising intangible capital, shrinking debt capacity, and the us corporate savings glut. NBER Working Paper.

Fama, E. F. and French, K. R. (1997). Industry costs of equity. Journal of Financial Economics, 43(2):153-193.

Fazzari, S., Hubbard, R. G., and Petersen, B. C. (1988). Financing constraints and corporate investment. Brookings Papers on Economic Activity, 1:141-206.

Gan, J. (2007). The real effects of asset market bubbles: Loan-and firm-level evidence of a lending channel. The Review of Financial Studies, 20:1941-1973.

Gao, X. and Ritter, J. R. (2010). The marketing of seasoned equity offerings. Journal of Financial Economics, 97(1):33-52.

Gustafson, M. T. and Iliev, P. (2017). The effects of removing barriers to equity issuance. Journal of Financial Economics, 124(3):580-598.

Harford, J. and Uysal, V. B. (2014). Bond market access and investment. Journal of Financial Economics, 112(2):147-163.

Hoberg, G., Phillips, G., and Prabhala, N. (2014). Product market threats, payouts, and financial flexibility. The Journal of Finance, 69(1):293-324.

Hsu, P.-H., Tian, X., and Xu, Y. (2014). Financial development and innovation: Crosscountry evidence. Journal of Financial Economics, 112(1):116-135.

Kerr, W. R. and Nanda, R. (2009). Democratizing entry: Banking deregulations, financing constraints, and entrepreneurship. Journal of Financial Economics, 94(1):124-149.

Khurana, I. K., Martin, X., and Pereira, R. (2006). Financial development and the cash flow sensitivity of cash. Journal of Financial and Quantitative Analysis, 41(4):787-808.

Kiyotaki, N. and Moore, J. (1997). Credit cycles. Journal of political economy, 105:211248.

Krishnan, K., Nandy, D. K., and Puri, M. (2014). Does financing spur small business productivity? evidence from a natural experiment. The Review of Financial Studies, 28(6):1768-1809.

Kumar, R. and Shome, D. K. (2008). The revival of shelf-registered corporate equity offerings. Journal of Corporate Finance, 14(1):32-50.

Lei, J., Qiu, J., and Wan, C. (2018). Asset tangibility, cash holdings, and financial development. Journal of Corporate Finance, 50:223-242.

Levine, R. and Zervos, S. (1998). Stock markets, banks, and economic growth. American economic review, pages 537-558.

Liberti, J. M. and Mian, A. R. (2010). Collateral spread and financial development. The Journal of Finance, 65(1):147-177. 
Love, I. (2003). Financial development and financing constraints: International evidence from the structural investment model. The Review of Financial Studies, 16(3):765-791.

Lyandres, E. and Palazzo, B. (2016). Cash holdings, competition, and innovation. Journal of Financial and Quantitative Analysis, 51(6):1823-1861.

Opler, T., Pinkowitz, L., Stulz, R., and Williamson, R. (1999). The determinants and implications of corporate cash holdings. Journal of Financial Economics, 52(1):3-46.

Tang, T. (2009). Information asymmetry and firms' credit market access: Evidence from moody's credit rating format refinement. Journal of Financial Economics, 93(2):325351.

Whited, T. M. (1992). Debt, liquidity constraints, and corporate investment: Evidence from panel data. The Journal of Finance, 47(4):1425-1460. 
Table 3.1: Summary statistics: comparison of key characteristics

This table presents the main firm characteristics split by firm treatment status in the pre and post shelf registration rule period. All sample filters described in Section 3.3 are applied in this table. All continuous variables are winsorized at the $1 \%$ level. Detailed description of the variables are in Table 3.10 .

\begin{tabular}{lcccccccc}
\hline & \multicolumn{3}{c}{ Pre-deregulation $(2002-2008)$} & \multicolumn{2}{c}{ Post-deregulation (2008-2012) } \\
\hline & \multicolumn{2}{c}{ Treated } & \multicolumn{2}{c}{ Nontreated } & \multicolumn{2}{c}{ Treated } & \multicolumn{2}{c}{ Nontreated } \\
& Mean & S.D & Mean & S.D & Mean & S.D & Mean & S.D \\
\hline \hline (a) Size variables & & & & & & & & \\
Ln(Public Float) & 34.10 & 17.15 & 112.35 & 20.17 & 34.42 & 16.84 & 112.8 & 20.69 \\
Firm Size & 3.88 & 1.13 & 6.22 & 2.10 & 4.11 & 1.18 & 6.68 & 2.07 \\
(b) Dependent variables & & & & & & & & \\
Cash & 0.26 & 0.29 & 0.25 & 0.29 & 0.27 & 0.3 & 0.24 & 0.27 \\
Investment & 0.045 & 0.07 & 0.06 & 0.08 & 0.041 & 0.07 & 0.06 & 0.08 \\
(c) Other controls & & & & & & & & \\
Asset Tangibility & 0.51 & 0.4 & 0.69 & 0.39 & 0.51 & 0.45 & 0.61 & 0.42 \\
Market-to-book & 1.97 & 1.75 & 2.24 & 1.7 & 1.68 & 1.52 & 2.09 & 1.65 \\
Cash Flow & -0.09 & 0.30 & 0.01 & 0.21 & -0.08 & 0.30 & 0.02 & 0.2 \\
Dividend Paying Firms (0/1) & 0.11 & 0.32 & 0.31 & 0.46 & 0.15 & 0.36 & 0.37 & 0.48 \\
Book Leverage & 0.18 & 0.22 & 0.20 & 0.21 & 0.16 & 0.21 & 0.21 & 0.21 \\
\hline
\end{tabular}


Table 3.2: Financing and cash-tangibility sensitivity

This table reports estimation results for the effect of deregulation on the sensitivity of cash to asset tangibility. The dependent variable, Cash, is the ratio of cash and marketable securities (CHE) to beginning period total assets (AT). Treated $(0 / 1)$ is one for a firm with a public float of less than $\$ 75$ million, otherwise zero. Post $(0 / 1)$ is one for periods after 2008, otherwise zero. Asset Tangibility is the ratio of net property, plant, and equipment (PPENT) to total book assets (AT). Columns 1 and 2 report baseline estimation results. Columns 3 and 4 reports results using a tighter public float of $\$ 25$ million to $\$ 125$ million. Columns 5 and 6 reports estimation results using a shorter sample period of 2005-2012. The variable of interest Asset Tangibility $\times$ Treated (0/1) $\times$ Post (0/1) tests for the impact of equity issuance deregulation on cash-tangibility sensitivity. All continuous variables are winsorized at the $1 \%$ level. Standard errors clustered by firm are reported in parentheses with less than $1 \%, 5 \%$, and $10 \%$ levels of statistical significance denoted by ***, $* *$, and $*$, respectively.

\begin{tabular}{|c|c|c|c|c|c|c|}
\hline & \multicolumn{2}{|c|}{ Baseline } & \multicolumn{2}{|c|}{$\begin{array}{c}\text { Dependent Variable - Cash } \\
\text { Tighter Public Float }\end{array}$} & \multicolumn{2}{|c|}{ Shorter Sample Period } \\
\hline & $(1)$ & $(2)$ & $(3)$ & $(4)$ & $(5)$ & $(6)$ \\
\hline Asset Tangibility & $\begin{array}{c}-0.1607^{* * *} \\
(0.0109)\end{array}$ & $\begin{array}{c}-0.1679^{* * *} \\
(0.0118)\end{array}$ & $\begin{array}{c}-0.1722^{* * *} \\
(0.0133)\end{array}$ & $\begin{array}{c}-0.1804^{* * *} \\
(0.0142)\end{array}$ & $\begin{array}{c}-0.1575^{* * *} \\
(0.0127)\end{array}$ & $\begin{array}{c}-0.1736^{* * *} \\
(0.0143)\end{array}$ \\
\hline Asset Tangibility $\times \operatorname{Treated}(0 / 1) \times \operatorname{Post}(0 / 1)$ & & $\begin{array}{c}0.0367^{* *} \\
(0.0177)\end{array}$ & & $\begin{array}{l}0.0375^{*} \\
(0.0203)\end{array}$ & & $\begin{array}{c}0.0431^{* *} \\
(0.0180)\end{array}$ \\
\hline Treated $(0 / 1) \times \operatorname{Post}(0 / 1)$ & & $\begin{array}{c}0.0022 \\
(0.0201)\end{array}$ & & $\begin{array}{l}-0.0376 \\
(0.0238)\end{array}$ & & $\begin{array}{l}-0.0276 \\
(0.0211)\end{array}$ \\
\hline Treated $(0 / 1)$ & & $\begin{array}{l}-0.0050 \\
(0.0110)\end{array}$ & & $\begin{array}{c}0.0123 \\
(0.0148)\end{array}$ & & $\begin{array}{l}-0.0082 \\
(0.0157)\end{array}$ \\
\hline Ln(Public Float) & $\begin{array}{l}-0.0005 \\
(0.0059)\end{array}$ & $\begin{array}{l}-0.0008 \\
(0.0078)\end{array}$ & $\begin{array}{c}0.0283^{* * *} \\
(0.0098)\end{array}$ & $\begin{array}{c}0.0342^{* *} \\
(0.0148)\end{array}$ & $\begin{array}{c}0.0208^{* *} \\
(0.0084)\end{array}$ & $\begin{array}{c}0.0151 \\
(0.0109)\end{array}$ \\
\hline Firm Size & $\begin{array}{c}0.0107^{* *} \\
(0.0049)\end{array}$ & $\begin{array}{c}0.0106 * * \\
(0.0049)\end{array}$ & $\begin{array}{c}0.0030 \\
(0.0063)\end{array}$ & $\begin{array}{c}0.0030 \\
(0.0063)\end{array}$ & $\begin{array}{l}-0.0018 \\
(0.0072)\end{array}$ & $\begin{array}{l}-0.0018 \\
(0.0072)\end{array}$ \\
\hline Market-to-book & $\begin{array}{c}0.0242^{* * *} \\
(0.0034)\end{array}$ & $\begin{array}{c}0.0241^{* * *} \\
(0.0034)\end{array}$ & $\begin{array}{c}0.0330 * * * \\
(0.0041)\end{array}$ & $\begin{array}{c}0.0329 * * * \\
(0.0041)\end{array}$ & $\begin{array}{c}0.0282^{* * *} \\
(0.0049)\end{array}$ & $\begin{array}{c}0.0280 * * * \\
(0.0049)\end{array}$ \\
\hline Cash Flow & $\begin{array}{c}0.0085 \\
(0.0529)\end{array}$ & $\begin{array}{c}0.0083 \\
(0.0530)\end{array}$ & $\begin{array}{c}-0.0837^{* * *} \\
(0.0222)\end{array}$ & $\begin{array}{c}-0.0829 * * * \\
(0.0223)\end{array}$ & $\begin{array}{c}-0.0813^{* * *} \\
(0.0221)\end{array}$ & $\begin{array}{c}-0.0816^{* * *} \\
(0.0221)\end{array}$ \\
\hline Dividend Paying Firms (0/1) & $\begin{array}{l}-0.0081 \\
(0.0542)\end{array}$ & $\begin{array}{l}-0.0074 \\
(0.0543)\end{array}$ & $\begin{array}{c}-0.0283^{* * *} \\
(0.0103)\end{array}$ & $\begin{array}{c}-0.0279 * * * \\
(0.0104)\end{array}$ & $\begin{array}{l}-0.0191^{*} \\
(0.0103)\end{array}$ & $\begin{array}{c}-0.0189^{*} \\
(0.0103)\end{array}$ \\
\hline Book Leverage & $\begin{array}{c}-0.3050^{* * *} \\
(0.0184)\end{array}$ & $\begin{array}{c}-0.3033^{* * *} \\
(0.0184)\end{array}$ & $\begin{array}{c}-0.3428 * * * \\
(0.0226)\end{array}$ & $\begin{array}{c}-0.3418^{* * *} \\
(0.0227)\end{array}$ & $\begin{array}{c}-0.3537^{* * *} \\
(0.0230)\end{array}$ & $\begin{array}{c}-0.3533^{* * *} \\
(0.0231)\end{array}$ \\
\hline Year Fixed Effects & Yes & Yes & Yes & Yes & Yes & Yes \\
\hline Industry Fixed Effects & Yes & Yes & Yes & Yes & Yes & Yes \\
\hline Observations & 7,195 & 7,195 & 4,892 & 4,892 & 4,404 & 4,404 \\
\hline$R^{2}$ & 0.4699 & 0.4703 & 0.4308 & 0.4314 & 0.4134 & 0.4143 \\
\hline
\end{tabular}


Table 3.3: Placebo: lower and upper public float cutoffs

This table reports placebo estimation results for the effect of deregulation on the sensitivity of cash to asset tangibility. Column 1 represents the lower placebo of public float between $\$ 10$ million to $\$ 70$ million with a cutoff of $\$ 40$ million. Column 2 represents the upper placebo of public float between $\$ 90$ million to $\$ 150$ million with a cutoff of $\$ 120$ million. In Columns 1 and 2 , Treated(0/1) is one for a firm with a public float of less than $\$ 40$ million and $\$ 120$ million respectively, otherwise zero. $\operatorname{Post}(0 / 1)$ is one for periods after 2008, otherwise zero. The dependent variable, Cash, is the ratio of cash and marketable securities (CHE) to beginning period total assets (AT). The variable of interest Asset Tangibility $\times$ Treated $(0 / 1) \times$ Post $(0 / 1)$ tests for the impact of equity issuance deregulation on cash-tangibility sensitivity. All continuous variables are winsorized at the $1 \%$ level. Standard errors clustered by firm are reported in parentheses with less than 1\%, $5 \%$, and $10 \%$ levels of statistical significance denoted by $* * *, * *$, and $*$, respectively.

\begin{tabular}{|c|c|c|}
\hline & $\begin{array}{c}\text { Dependent V } \\
(1) \\
\text { Lower Placebo }\end{array}$ & $\begin{array}{c}\text { riable }- \text { Cash } \\
(2) \\
\text { Upper Placebo }\end{array}$ \\
\hline Asset Tangibility & $\begin{array}{c}-0.1572^{* * *} \\
(0.0136)\end{array}$ & $\begin{array}{c}-0.1824^{* * *} \\
(0.0224)\end{array}$ \\
\hline Asset Tangibility $\times \operatorname{Treated}(0 / 1) \times \operatorname{Post}(0 / 1)$ & $\begin{array}{c}0.0274 \\
(0.0226)\end{array}$ & $\begin{array}{c}0.0150 \\
(0.0373)\end{array}$ \\
\hline $\operatorname{Treated}(0 / 1) \times \operatorname{Post}(0 / 1)$ & $\begin{array}{c}-0.0029 \\
(0.0254)\end{array}$ & $\begin{array}{c}0.0805^{* *} \\
(0.0398)\end{array}$ \\
\hline Treated $(0 / 1)$ & $\begin{array}{c}-0.0265^{*} \\
(0.0147)\end{array}$ & $\begin{array}{c}-0.0200 \\
(0.0313)\end{array}$ \\
\hline Ln(Public Float) & $\begin{array}{c}-0.0154 \\
(0.0130)\end{array}$ & $\begin{array}{c}-0.0157 \\
(0.0949)\end{array}$ \\
\hline Firm Size & $\begin{array}{c}0.0152^{* *} \\
(0.0059)\end{array}$ & $\begin{array}{c}-0.0042 \\
(0.0101)\end{array}$ \\
\hline Market-to-book & $\begin{array}{c}0.0263^{* * *} \\
(0.0043)\end{array}$ & $\begin{array}{c}0.0245^{* * *} \\
(0.0074)\end{array}$ \\
\hline Cash Flow & $\begin{array}{c}0.0640 \\
(0.0619)\end{array}$ & $\begin{array}{c}0.0633 \\
(0.1041)\end{array}$ \\
\hline Dividend Paying Firms (0/1) & $\begin{array}{c}-0.0081 \\
(0.0635)\end{array}$ & $\begin{array}{c}0.0930 \\
(0.1022)\end{array}$ \\
\hline Book Leverage & $\begin{array}{c}-0.3106^{* * *} \\
(0.0235)\end{array}$ & $\begin{array}{c}-0.3029^{* * *} \\
(0.0338)\end{array}$ \\
\hline Year Fixed Effects & Yes & Yes \\
\hline Industry Fixed Effects & Yes & Yes \\
\hline Observations & 3,961 & 3,234 \\
\hline$R^{2}$ & 0.4161 & 0.5551 \\
\hline
\end{tabular}




\section{Table 3.4: Information asymmetry and tech intensiveness}

This table reports estimation results for the effect of deregulation on the sensitivity of cash to asset tangibility conditional upon firm opaqueness and tech intensiveness. The dependent variable, Cash, is the ratio of cash and marketable securities (CHE) to beginning period total assets (AT). Treated $(0 / 1)$ is one for a firm with a public float of less than $\$ 75$ million, otherwise zero. Post(0/1) is one for periods after 2008, otherwise zero. Asset Tangibility is the ratio of net property, plant, and equipment (PPENT) to total book assets (AT). Firm Age is the natural log of the number of years a firm has been listed in the Compustat. Tech Intensiveness is the annual gross growth in R\&D expenditure. See Table 3.10 for all other variable definitions. All continuous variables are winsorized at the $1 \%$ level. Standard errors clustered by firm are reported in parentheses with less than $1 \%, 5 \%$, and $10 \%$ levels of statistical significance denoted by $* * *, * *$, and $*$, respectively.

\begin{tabular}{|c|c|c|c|c|}
\hline & \multicolumn{4}{|c|}{ Dependent Variable - Cash } \\
\hline & \multicolumn{2}{|c|}{ Firm Age } & \multicolumn{2}{|c|}{ Tech Intensiveness } \\
\hline & Young & Old & Low Tech & High Tech \\
\hline Asset Tangibility & $\begin{array}{c}-0.2023^{* * *} \\
(0.0180)\end{array}$ & $\begin{array}{c}-0.1451^{* * *} \\
(0.0165)\end{array}$ & $\begin{array}{c}-0.1429 * * * \\
(0.0121)\end{array}$ & $\begin{array}{c}-0.2582^{* * *} \\
(0.0210)\end{array}$ \\
\hline Asset Tangibility $\times$ Treated $(0 / 1) \times \operatorname{Post}(0 / 1)$ & $\begin{array}{c}0.0938 * * * \\
(0.0302)\end{array}$ & $\begin{array}{l}-0.0063 \\
(0.0260)\end{array}$ & $\begin{array}{c}0.0215 \\
(0.0181)\end{array}$ & $\begin{array}{l}0.0753^{*} \\
(0.0421)\end{array}$ \\
\hline $\operatorname{Treated}(0 / 1) \times \operatorname{Post}(0 / 1)$ & $\begin{array}{l}-0.0589^{*} \\
(0.0310)\end{array}$ & $\begin{array}{l}-0.0004 \\
(0.0296)\end{array}$ & $\begin{array}{l}-0.0420^{*} \\
(0.0219)\end{array}$ & $\begin{array}{l}-0.0090 \\
(0.0341)\end{array}$ \\
\hline Treated $(0 / 1)$ & $\begin{array}{c}0.0081 \\
(0.0181)\end{array}$ & $\begin{array}{l}-0.0172 \\
(0.0168)\end{array}$ & $\begin{array}{c}0.0193 \\
(0.0137)\end{array}$ & $\begin{array}{l}-0.0252 \\
(0.0200)\end{array}$ \\
\hline Ln(Public Float) & $\begin{array}{c}0.0186 \\
(0.0135)\end{array}$ & $\begin{array}{c}0.0019 \\
(0.0113)\end{array}$ & $\begin{array}{c}0.0119 \\
(0.0094)\end{array}$ & $\begin{array}{c}0.0047 \\
(0.0137)\end{array}$ \\
\hline Firm Size & $\begin{array}{c}0.0089 \\
(0.0079)\end{array}$ & $\begin{array}{c}0.0007 \\
(0.0084)\end{array}$ & $\begin{array}{l}-0.0049 \\
(0.0058)\end{array}$ & $\begin{array}{c}0.0275^{* * *} \\
(0.0081)\end{array}$ \\
\hline Market-to-book & $\begin{array}{c}0.0299^{* * *} \\
(0.0047)\end{array}$ & $\begin{array}{c}0.0388^{* * *} \\
(0.0071)\end{array}$ & $\begin{array}{c}0.0360^{* * *} \\
(0.0052)\end{array}$ & $\begin{array}{c}0.0297^{* * *} \\
(0.0046)\end{array}$ \\
\hline Cash Flow & $\begin{array}{c}-0.0656^{* *} \\
(0.0272)\end{array}$ & $\begin{array}{c}-0.0825^{* *} \\
(0.0372)\end{array}$ & $\begin{array}{l}-0.0333 \\
(0.0228)\end{array}$ & $\begin{array}{c}-0.1185^{* * *} \\
(0.0251)\end{array}$ \\
\hline Dividend Paying Firms (0/1) & $\begin{array}{c}-0.0695^{* * *} \\
(0.0172)\end{array}$ & $\begin{array}{c}0.0015 \\
(0.0116)\end{array}$ & $\begin{array}{l}-0.0075 \\
(0.0100)\end{array}$ & $\begin{array}{c}-0.0588^{* * *} \\
(0.0168)\end{array}$ \\
\hline Book Leverage & $\begin{array}{c}-0.3448^{* * *} \\
(0.0271)\end{array}$ & $\begin{array}{c}-0.3168^{* * *} \\
(0.0304)\end{array}$ & $\begin{array}{c}-0.2918^{* * *} \\
(0.0219)\end{array}$ & $\begin{array}{c}-0.4030^{* * *} \\
(0.0285)\end{array}$ \\
\hline Year Fixed Effects & Yes & Yes & Yes & Yes \\
\hline Industry Fixed Effects & Yes & Yes & Yes & Yes \\
\hline Observations & 3,316 & 3,185 & 3,412 & 3,150 \\
\hline$R^{2}$ & 0.4139 & 0.3916 & 0.4189 & 0.4034 \\
\hline
\end{tabular}


Table 3.5: Financing and the investment-cash flow sensitivity

This table reports estimation results for the investment-cash flow sensitivity. Column 1 directly measures the investment-cash flow sensitivity. Column 2 test whether the investment-cash flow sensitivity is affected by the financial deregulation of equity issuance. All continuous variables are winsorized at the 1\% level. Standard errors clustered by firm are reported in parentheses with less than $1 \%, 5 \%$, and $10 \%$ levels of statistical significance denoted by $* * *, * *$, and ${ }^{*}$, respectively.

\begin{tabular}{|c|c|c|}
\hline & \multicolumn{2}{|c|}{$\begin{array}{l}\text { Dependent Variable - Investment } \\
(1)\end{array}$} \\
\hline Cash Flow & $\begin{array}{c}0.0323^{* * *} \\
(0.0031)\end{array}$ & $\begin{array}{c}0.0329 * * * \\
(0.0032)\end{array}$ \\
\hline Cash Flow $\times \operatorname{Treated}(0 / 1) \times \operatorname{Post}(0 / 1)$ & & $\begin{array}{c}-0.0286^{* * *} \\
(0.0105)\end{array}$ \\
\hline $\operatorname{Treated}(0 / 1) \times \operatorname{Post}(0 / 1)$ & & $\begin{array}{c}-0.0021 \\
(0.0023)\end{array}$ \\
\hline Treated $(0 / 1)$ & & $\begin{array}{c}-0.0032^{* *} \\
(0.0014)\end{array}$ \\
\hline Market-to-book & $\begin{array}{c}0.0042^{* * *} \\
(0.0004)\end{array}$ & $\begin{array}{c}0.0041^{* * *} \\
(0.0004)\end{array}$ \\
\hline Cash & $\begin{array}{c}0.0133^{* * *} \\
(0.0027)\end{array}$ & $\begin{array}{c}0.0131^{* * *} \\
(0.0027)\end{array}$ \\
\hline Book Leverage & $\begin{array}{c}0.0140^{* * *} \\
(0.0035)\end{array}$ & $\begin{array}{c}0.0139 * * * \\
(0.0035)\end{array}$ \\
\hline Year Fixed Effects & Yes & Yes \\
\hline Industry Fixed Effects & Yes & Yes \\
\hline Observations & 7,195 & 7,195 \\
\hline$R^{2}$ & 0.2899 & 0.2905 \\
\hline
\end{tabular}


Table 3.6: Financing and investment-tangibility sensitivity

This table reports estimation results for the effect of equity issuance deregulation on the sensitivity of investment to asset tangibility. The dependent variable, Investment, is defined as the ratio of capital expenditure to beginning of period total assets.

Treated $(0 / 1)$ is one for a firm with a public float of less than $\$ 75$ million, otherwise zero. Post(0/1) is one for periods after 2008, otherwise zero. Asset Tangibility is the ratio of net property, plant, and equipment (PPENT) to total book assets (AT). In Column 1, the variable of interest, Treated $(0 / 1) \times \operatorname{Post}(0 / 1)$, tests for the effect of the deregulation on investment. Column 2 reports regression estimates of the direct effect of tangibility on investment. In Column 3, Asset Tangibility $\times \operatorname{Treated}(0 / 1) \times \operatorname{Post}(0 / 1)$ tests for the impact of equity issuance deregulation on investment-tangibility sensitivity. All continuous variables are winsorized at the $1 \%$ level. Standard errors clustered by firm are reported in parentheses with less than 1\%,5\%, and $10 \%$ levels of statistical significance denoted by $* * *, * *$, and $*$, respectively.

Dependent Variable - Investment

(1)

(2)

\begin{tabular}{|c|c|c|c|}
\hline Asset Tangibility & & $\begin{array}{c}0.0396^{* * *} \\
(0.0030)\end{array}$ & $\begin{array}{c}0.0444^{* * *} \\
(0.0032)\end{array}$ \\
\hline Asset Tangibility $\times \operatorname{Treated}(0 / 1) \times \operatorname{Post}(0 / 1)$ & & & $\begin{array}{c}-0.0208^{* * *} \\
(0.0066)\end{array}$ \\
\hline $\operatorname{Treated}(0 / 1) \times \operatorname{Post}(0 / 1)$ & $\begin{array}{c}0.0091^{* *} \\
(0.0037)\end{array}$ & & $\begin{array}{c}0.0213^{* * *} \\
(0.0043)\end{array}$ \\
\hline Treated $(0 / 1)$ & $\begin{array}{c}-0.0006 \\
(0.0029)\end{array}$ & & $\begin{array}{c}-0.0004 \\
(0.0028)\end{array}$ \\
\hline Ln(Public Float) & $\begin{array}{c}0.0084^{* * *} \\
(0.0017)\end{array}$ & $\begin{array}{c}0.0084^{* * *} \\
(0.0014)\end{array}$ & $\begin{array}{c}0.0097^{* * *} \\
(0.0017)\end{array}$ \\
\hline Firm Size & $\begin{array}{c}-0.0055^{* * *} \\
(0.0013)\end{array}$ & $\begin{array}{c}-0.0046^{* * *} \\
(0.0012)\end{array}$ & $\begin{array}{c}-0.0046^{* * *} \\
(0.0012)\end{array}$ \\
\hline Market-to-book & $\begin{array}{c}0.0018^{* *} \\
(0.0007)\end{array}$ & $\begin{array}{c}0.0022^{* * *} \\
(0.0007)\end{array}$ & $\begin{array}{c}0.0022^{* * *} \\
(0.0007)\end{array}$ \\
\hline Cash Flow & $\begin{array}{c}0.0422^{* * *} \\
(0.0107)\end{array}$ & $\begin{array}{c}0.0440^{* * * *} \\
(0.0108)\end{array}$ & $\begin{array}{c}0.0451^{* * *} \\
(0.0106)\end{array}$ \\
\hline Dividend Paying Firms (0/1) & $\begin{array}{c}-0.0021 \\
(0.0114)\end{array}$ & $\begin{array}{c}-0.0085^{* * *} \\
(0.0113)\end{array}$ & $\begin{array}{c}-0.0087^{* * *} \\
(0.0112)\end{array}$ \\
\hline Book Leverage & $\begin{array}{c}0.0186^{* * *} \\
(0.0051)\end{array}$ & $\begin{array}{c}0.0091^{*} \\
(0.0049)\end{array}$ & $\begin{array}{l}0.0089^{*} \\
(0.0048)\end{array}$ \\
\hline Year Fixed Effects & Yes & Yes & Yes \\
\hline Industry Fixed Effects & Yes & Yes & Yes \\
\hline Observations & 7,195 & 7,195 & 7,195 \\
\hline$R^{2}$ & 0.2605 & 0.2922 & 0.2954 \\
\hline
\end{tabular}


Table 3.7: Financing and investment efficiency

This table reports estimation results for the effect of equity issuance deregulation on investment efficiency. The dependent variable in all columns is investment efficiency. The level of investment from the investment model of Eq.(1.4) are based on sales growth in Column 1, Tobin's Q in Column 2, and both sales growth and Tobin's Q in Column 3. Treated $(0 / 1)$ is one for a firm with a public float of less than $\$ 75$ million, otherwise zero. Post(0/1) is one for periods after 2008, otherwise zero. All continuous variables are winsorized at the $1 \%$ level. Standard errors clustered by firm are reported in parentheses with less than 1\%,5\%, and $10 \%$ levels of statistical significance denoted by $* * *, * *$, and $*$, respectively.

\begin{tabular}{|c|c|c|c|}
\hline & \multicolumn{3}{|c|}{ Dependent Variable - Investment Efficiency } \\
\hline & Sales Growth & Tobin's $Q$ & Sales Growth and Tobin's $Q$ \\
\hline \multirow[t]{2}{*}{$\overline{\operatorname{Treated}(0 / 1) \times \operatorname{Post}(0 / 1)}$} & $0.0187^{* *}$ & $0.0210^{* * *}$ & $0.0230^{* * *}$ \\
\hline & $(0.0077)$ & $(0.0074)$ & $(0.0074)$ \\
\hline \multirow[t]{2}{*}{ Treated $(0 / 1)$} & $-0.0114^{* *}$ & -0.0099 & -0.0075 \\
\hline & $(0.0054)$ & $(0.0051)$ & $(0.0051)$ \\
\hline \multirow[t]{2}{*}{ Ln(Public Float) } & -0.0046 & $-0.0085^{* *}$ & $-0.0085^{* *}$ \\
\hline & $(0.0038)$ & $(0.0037)$ & $(0.0037)$ \\
\hline \multirow[t]{2}{*}{ Firm Size } & 0.0037 & $0.0077^{* * *}$ & $0.0077^{* * *}$ \\
\hline & $(0.0024)$ & $(0.0023)$ & $(0.0023)$ \\
\hline \multirow[t]{2}{*}{ Market-to-book } & $-0.0058^{* * *}$ & 0.0011 & 0.0011 \\
\hline & $(0.0019)$ & $(0.0017)$ & $(0.0017)$ \\
\hline \multirow[t]{2}{*}{ Cash Flow } & $0.1504^{* * *}$ & $0.1385^{* * *}$ & $0.1385^{* * *}$ \\
\hline & $(0.0112)$ & $(0.0115)$ & $(0.0115)$ \\
\hline \multirow[t]{2}{*}{ Dividend Paying Firms (0/1) } & -0.0020 & -0.0019 & -0.0019 \\
\hline & $(0.0037)$ & $(0.0035)$ & $(0.0035)$ \\
\hline \multirow[t]{2}{*}{ Book Leverage } & -0.0004 & 0.0041 & 0.0041 \\
\hline & $(0.0120)$ & $(0.0106)$ & $(0.0106)$ \\
\hline Year Fixed Effects & Yes & Yes & Yes \\
\hline Industry Fixed Effects & Yes & Yes & Yes \\
\hline Observations & 6,866 & 6,866 & 6,866 \\
\hline$R^{2}$ & 0.3712 & 0.3291 & 0.329 \\
\hline
\end{tabular}




\section{Table 3.8: Financing and fixed asset growth}

This table reports estimation results for the effect of the stock issuance deregulation on fixed asset structure. In Columns 1 and 2, change in fixed assets is defined as the change in firms' property, plant and equipment (PPEGT), and scaled by beginning of period total assets (AT). In Columns 3 and 4, change in net fixed assets is change in firms' net property, plant and equipment (PPENT), and scaled by beginning of period total assets (AT). Treated $(0 / 1)$ is one for a firm with a public float of less than $\$ 75$ million, otherwise zero. Post(0/1) is one for periods after 2008, otherwise zero. All continuous variables are winsorized at the $1 \%$ level. Standard errors clustered by firm are reported in parentheses with less than $1 \%, 5 \%$, and $10 \%$ levels of statistical significance denoted by ${ }^{* *},{ }^{* *}$, and ${ }^{*}$, respectively.

\begin{tabular}{lcccc}
\hline & \multicolumn{2}{c}{ Fixed Asset Growth } & \multicolumn{2}{c}{ Net Fixed Asset Growth } \\
& $(1)$ & $(2)$ & $(3)$ & $(4)$ \\
\hline \hline Treated(0/1)× Post $(0 / 1)$ & $0.0172^{* * *}$ & $0.0452^{* * *}$ & $0.0140^{* * *}$ & $0.0563^{* * *}$ \\
& $(0.0064)$ & $(0.0149)$ & $(0.0052)$ & $(0.0208)$ \\
Treated(0/1) & 0.0048 & 0.0022 & 0.0046 & 0.0020 \\
& $(0.0049)$ & $(0.0117)$ & $(0.0037)$ & $(0.0161)$ \\
Ln(Public Float) & $0.0061^{*}$ & $0.0149^{*}$ & $0.0042^{*}$ & $0.0189^{*}$ \\
& $(0.0033)$ & $(0.0080)$ & $(0.0024)$ & $(0.0110)$ \\
Firm Size & $0.0047^{* *}$ & $0.0161^{* * *}$ & $0.0042^{* *}$ & $0.0227^{* * *}$ \\
& $(0.0023)$ & $(0.0052)$ & $(0.0018)$ & $(0.0071)$ \\
Market-to-book & $0.0028^{* *}$ & $0.0142^{* * *}$ & $0.0024^{* * *}$ & $0.0236^{* * *}$ \\
& $(0.0012)$ & $(0.0029)$ & $(0.0009)$ & $(0.0046)$ \\
Cash Flow & $0.0614^{* * *}$ & $0.1907^{* * *}$ & $0.0294^{*}$ & $0.1945^{* *}$ \\
& $(0.0227)$ & $(0.0593)$ & $(0.0166)$ & $(0.0838)$ \\
Dividend Paying Firms $(0 / 1)$ & 0.0029 & -0.0120 & 0.0048 & $0.0281^{* *}$ \\
& $(0.0230)$ & $(0.0591)$ & $(0.0165)$ & $(0.0854)$ \\
Book Leverage & $0.0171^{*}$ & -0.0051 & 0.0043 & -0.0169 \\
& $(0.0090)$ & $(0.0188)$ & $(0.0071)$ & $(0.0266)$ \\
Year Fixed Effects & Yes & Yes & Yes & Yes \\
Industry Fixed Effects & Yes & Yes & Yes & Yes \\
Observations & 7,185 & 7,165 & 7,212 & 7,186 \\
$R^{2}$ & 0.2616 & 0.1252 & 0.2463 & 0.1871 \\
\hline
\end{tabular}


Table 3.9: Robustness check: financing and sensitivity of cash and investment to asset tangibility

This table reports the re-estimated baseline equation for the effect of deregulation on the sensitivities of cash and investment to asset tangibility but additionally controls for firm fixed effects. The dependent variable in Columns 1 and 2 is Cash and Investment in Columns 3 and 4 , Investment. Treated(0/1) is one for a firm with a public float of less than $\$ 75$ million, otherwise zero. Post $(0 / 1)$ is one for periods after 2008, otherwise zero. Columns 1 and 3 report regression estimates of the direct effect of tangibility on cash and investment. In Columns 2 and 4 , Asset Tangibility $\times$ Treated $(0 / 1) \times$ Post $(0 / 1)$ tests for the impact of deregulation on the sensitivities of cash and investment to asset tangibility. All continuous variables are winsorized at the 1\% level. Standard errors clustered by firm are reported in parentheses with less than $1 \%, 5 \%$, and $10 \%$ levels of statistical significance denoted by $* * *, * *$, and *, respectively.

\begin{tabular}{|c|c|c|c|c|}
\hline & \multicolumn{2}{|c|}{ Cash } & \multicolumn{2}{|c|}{ Investment } \\
\hline & $(1)$ & $(2)$ & $(3)$ & $(4)$ \\
\hline Asset Tangibility & $\begin{array}{c}-0.2628^{* * *} \\
(0.0291)\end{array}$ & $\begin{array}{c}-0.2752^{* * *} \\
(0.0288)\end{array}$ & $\begin{array}{c}0.0330 * * * \\
(0.0067)\end{array}$ & $\begin{array}{c}0.0377^{* * *} \\
(0.0068)\end{array}$ \\
\hline Asset Tangibility $\times$ Treated $(0 / 1) \times \operatorname{Post}(0 / 1)$ & & $\begin{array}{c}0.0422^{*} \\
(0.0219)\end{array}$ & & $\begin{array}{c}-0.0145^{* *} \\
(0.0065)\end{array}$ \\
\hline Treated $(0 / 1) \times \operatorname{Post}(0 / 1)$ & & $\begin{array}{l}-0.0069 \\
(0.0223)\end{array}$ & & $\begin{array}{c}0.0132^{* * *} \\
(0.0043)\end{array}$ \\
\hline Treated $(0 / 1)$ & & $\begin{array}{c}0.0065 \\
(0.0108)\end{array}$ & & $\begin{array}{l}-0.0020 \\
(0.0020)\end{array}$ \\
\hline Ln(Public Float) & $\begin{array}{c}-0.0197^{* * *} \\
(0.0071)\end{array}$ & $\begin{array}{r}-0.0146^{*} \\
(0.0088)\end{array}$ & $\begin{array}{c}0.0080^{* * * *} \\
(0.0013)\end{array}$ & $\begin{array}{c}0.0078^{* * *} * \\
(0.0016)\end{array}$ \\
\hline Firm Size & $\begin{array}{c}0.0556^{* * *} \\
(0.0128)\end{array}$ & $\begin{array}{c}0.0550^{* * *} \\
(0.0129)\end{array}$ & $\begin{array}{c}0.0018 \\
(0.0023)\end{array}$ & $\begin{array}{c}0.0022 \\
(0.0022)\end{array}$ \\
\hline Market-to-book & $\begin{array}{c}0.0239^{* * *} * \\
(0.0042)\end{array}$ & $\begin{array}{c}0.0238^{* * *} \\
(0.0042)\end{array}$ & $\begin{array}{c}0.0009 \\
(0.0006)\end{array}$ & $\begin{array}{c}0.0010 \\
(0.0006)\end{array}$ \\
\hline Cash Flow & $\begin{array}{c}0.2846^{* * * *} \\
(0.0321)\end{array}$ & $\begin{array}{c}0.2842^{* * * *} \\
(0.0320)\end{array}$ & $\begin{array}{l}-0.0046 \\
(0.0044)\end{array}$ & $\begin{array}{l}-0.0043 \\
(0.0044)\end{array}$ \\
\hline Dividend Paying Firms (0/1) & $\begin{array}{c}0.0070 \\
(0.0124)\end{array}$ & $\begin{array}{c}0.0088 \\
(0.0124)\end{array}$ & $\begin{array}{c}0.0071^{* * *} \\
(0.0025)\end{array}$ & $\begin{array}{c}0.0067^{* * *} \\
(0.0025)\end{array}$ \\
\hline Book Leverage & $\begin{array}{c}-0.1799^{* * *} \\
(0.0349)\end{array}$ & $\begin{array}{c}-0.1808^{* * *} \\
(0.0350)\end{array}$ & $\begin{array}{l}-0.0047 \\
(0.0060)\end{array}$ & $\begin{array}{l}-0.0042 \\
(0.0060)\end{array}$ \\
\hline Year Fixed Effects & Yes & Yes & Yes & Yes \\
\hline Firm Fixed Effects & Yes & Yes & Yes & Yes \\
\hline Observations & 7,195 & 7,195 & 7,195 & 7,195 \\
\hline$R^{2}$ & 0.2004 & 0.2019 & 0.0363 & 0.0404 \\
\hline
\end{tabular}


Table 3.10: Variable definitions

This table provides the definition of the key variables used. Accounting data are from Compustat.

\begin{tabular}{|c|c|}
\hline Variable & Definition \\
\hline Treated $(0 / 1)$ & $\begin{array}{l}\text { One for a firm with a public float of less than } \$ 75 \text { million, oth- } \\
\text { erwise zero }\end{array}$ \\
\hline $\operatorname{Post}(0 / 1)$ & $\begin{array}{l}\text { One for periods after the amendment of the public float require- } \\
\text { ment in } 2008 \text {, otherwise zero }\end{array}$ \\
\hline Investment & Ratio of capital expenditure to beginning of period total assets \\
\hline Ln(Public Float) & Natural logarithm of public float \\
\hline Firm Size & Natural logarithm of total book assets \\
\hline Market-to-book & $\begin{array}{l}\text { Ratio of total book assets (AT) less the book value of common } \\
\text { equity (CEQ) plus the total market value of equity }(\mathrm{CSHO} \times \\
\left.P R C C_{C}\right) \text { all divided by the total book assets }(\mathrm{AT})\end{array}$ \\
\hline Asset Tangibility & $\begin{array}{l}\text { Ratio of net property, plant, and equipment (PPENT) to total } \\
\text { book assets (AT) }\end{array}$ \\
\hline RED Expenditure & $\begin{array}{l}\text { Ratio of research and development expense (XRD) to total book } \\
\text { assets (AT) }\end{array}$ \\
\hline Cash & $\begin{array}{l}\text { Cash and marketable securities (CHE) scaled by total book as- } \\
\text { sets (AT) }\end{array}$ \\
\hline Net Cash & Ratio of cash to total assets minus cash \\
\hline Cash Flow & $\begin{array}{l}\text { Earnings after interest, dividends and taxes, but before depreci- } \\
\text { ation (OIBDP) less interest (XINT), income taxes (TXT), and } \\
\text { dividends (DVC), all divided by book assets (AT) }\end{array}$ \\
\hline Book Leverage & Ratio of total book debt (DLC+DLTT) to total book assets (AT) \\
\hline Dividend Paying Firms (0/1) & $\begin{array}{l}\text { One in the year a firm pays dividend and zero otherwise; set to } \\
\text { zero if missing }\end{array}$ \\
\hline Firm Age & $\begin{array}{l}\text { Natural Logarithm of the number of years a firm has been listed } \\
\text { in the merged CRSP/Compustat database }\end{array}$ \\
\hline
\end{tabular}




\section{Conclusion}

This thesis is composed of three self-contained essays. In the first essay, we show that the increase in ex ante risk of incurring future costs related to data breaches due to mandatory data breach disclosure laws compel corporations to hold on to more cash as a precautionary motive. Also, we find that breached firms, in particular, severely breached firms, increase their cash holdings in response to the data breach incident. In other words, severely breached firms face the need to build up more cash. An examination of the possible underlying mechanisms through which firms build up cash after a data breach incident shows that breached firms decrease their external finance and investment.

In the second essay, we extend the literature about data breaches and corporate finance by examining whether credit risk perceptions change towards firms that experience data breaches relative to non-breached firms. Data breaches may affect firms' future performance, financial stability, and risk profiles, thus potentially resulting in increased credit risk. Using firm-level credit ratings and credit default swap (CDS) spreads to proxy for credit risk, we find that data breaches lead to increases in firm credit risk. Consistent with the notion that credit rating agencies view data breach risk exposure as a credit risk increasing event, we find that in the event of a data breach incident the predicted percentage of a firm being rated A decreases by $33.56 \%$ and the probability of a rating downgrade increases by $13 \%$. Firms exposed to data breaches are more likely to experience an increase in the CDS spread of traded bonds. Also, firms who suffer data breaches report lower sales and ROA, experience an increase in financial distress, and 
conditional on a data breach incident, the likelihood of a future data breach increases. Lastly, these effects are magnified for firms with low-interest coverage ratios.

Prior research shows that asset tangibility substantially impacts firms' cash levels and investment. In the third essay, using the financial deregulation of seasoned equity issuance in the U.S. as an exogenous shock to access to equity markets, I investigate the influence of financing on the dependence of cash and investment on asset tangibility. I show that improved access to financing dampens the sensitivity of cash and investment to asset tangibility, and promotes investment and firm growth. This provides evidence that public firms even in well-developed financial markets such as the U.S. benefit from financial deregulation that removes barriers to external equity financing, shedding light on the role of financial markets in fostering growth. 